\title{
Approche géométrique des joints de grains. Intérêt et limite
}

\author{
L. Priester \\ Laboratoire de Métallurgie Physique (*), Université Paris-Sud, 91405 Orsay, France \\ (Reçu le 3 octobre 1979, révisé le 11 janvier 1980, accepté le 15 janvier 1980)
}

\begin{abstract}
Résumé. - Après l'exposé des principaux concepts du modèle géométrique des joints de grains, une tentative est faite pour dégager dans quelle mesure ce modèle permet de décrire diverses structures interfaciales, dans le cas des matériaux de symétrie cubique, et de comprendre certaines réactions du joint avec la matrice. Quelques bases nécessaires à l'étude en microscopie électronique en transmission de ces structures et réactions sont alors données. Puis, un effort de synthèse sur les applications de l'approche géométrique à la compréhension des propriétés des interfaces est entrepris, en comparaison à l'apport du modèle énergétique. Il ressort de cette analyse que les démarches actuelles pour la connaissance du joint de grains sont assez similaires à celles faites, dans le passé, pour la connaissance du monocristal. En particulier, il semble que le modèle récent d'arrangement de groupements polyédriques d'atomes dans l'interface, analogue à l'empilement cristallin plus ou moins compact dans la matrice, soit actuellement le plus satisfaisant et laisse entrevoir une meilleure compréhension des propriétés interfaciales.
\end{abstract}

\begin{abstract}
The main concepts of the geometrical approach of grain boundaries are first given. Then an attempt is made to see how this model can describe various intergranular structures in cubic materials and some reactions of grain boundary with the neighbouring crystals. Some basis to perform electron microscopy studies of these structures and reactions are then given. We try to summarize the different applications of the geometrical approach of grain boundaries to their properties and to compare these possibilities to those of the energetical model. It is pointed out that the present approach to the knowledge of the grain boundary is quite similar to that in which the crystal was studied previously. Particularly, it is likely that the recent model of packing of polyhedra in the boundary, similar to the more or less close packed crystalline structure of grain, can satisfactorily explain most properties of grain boundaries.
\end{abstract}

1. Introduction. - Pour étudier, même d'un point de vue macroscopique, les réactions du métal aux diverses sollicitations thermiques, mécaniques ou chimiques (processus de recristallisation - transformation de phases - corrosion...), le métallurgiste ne peut faire abstraction de la connaissance de la structure de la matière à l'échelle submicroscopique. En effet, la plupart des propriétés métalliques s'expliquent en termes de défauts (ponctuels, linéaires ou plans) par rapport à un état parfait ; cet état est décrit géométriquement et énergétiquement : c'est l'ordre périodique des atomes correspondant à l'énergie minimale.

Cependant, les résultats plus récents dans les études des métaux et alliages ont mis en évidence le rôle essentiel des interfaces cristallines dans tous les mécanismes de réaction du matériau. On ne peut être que frappé alors par la contradiction qui consiste à continuer à s'appuer sur la structure et les propriétés du monocristal ou bon matériau en ignorant l'organisation des régions dites de mauvais matériau que

$\left({ }^{*}\right)$ Associé au C.N.R.S. constituent les joints de grains. En effet, les propriétés d'emploi des matériaux sont en général attachées à la forme polycristalline, ce qui implique le rôle fondamental des interfaces.

Or, depuis une dizaine d'années, des progrès importants ont été apportés dans la connaissance de la structure des joints de grains aussi bien par l'approche géométrique que par l'approche énergétique (ou analytique $\left({ }^{1}\right)$ ). Les études restent, pour la plupart, très théoriques et semblent un domaine encore limité aux physiciens de l'état solide. Cependant, en dépit des difficultés énormes que cela représente actuellement, la nécessité de prendre en compte les modèles, même approchés, des joints de grains est maintenant à l'ordre du jour pour les métallurgistes. L'étude de la structure des joints de grains ne peut être une fin en soi mais doit constituer le point de départ d'une meilleure compréhension des comportements des matériaux.

L'objet de cet article est, tout d'abord, de résumer les

( $\left.{ }^{1}\right)$ Comme l'approche géométrique s'appuie sur certaines considérations énergétiques, il peut sembler préférable d'utiliser pour les méthodes de simulation à l'ordinateur le terme analytique. 
principaux concepts concernant la structure des joints de grains en se basant essentiellement sur la description géométrique qui, malgré ses limites, est actuellement la plus pratique, ce qui ne signifie pas la mieux appropriée (\$ 5) pour l'étude des joints réels. Ces concepts sont ensuite appliqués à divers cas de structures de joints de grains de métaux cubiques étudiés par microscopie électronique en transmission; les méthodes d'étude sont alors décrites. Enfin, après avoir évoqué brièvement les principes de l'approche analytique, nous tentons de dégager les limites de l'un et l'autre modèle pour l'étude de certaines propriétés des joints de grains.

2. Structure des joints de grains. - La réalité du joint de grain consiste le plus vraisemblablement en un arrangement d'atomes que l'expérience ne permet pas d'atteindre actuellement. Cependant, il est possible de donner une description géométrique de l'organisation idéale des deux cristaux voisins interpénétrés au niveau du joint, sans perdre de vue que ce biréseau n'est pas la réalité intergranulaire et ne constitue qu'un outil ou un guide. Cette description est basée sur l'hypothèse qu'une situation énergétique favorable est associée à l'existence d'un invariant c'est-à-dire d'un ordre commun aux deux cristaux.

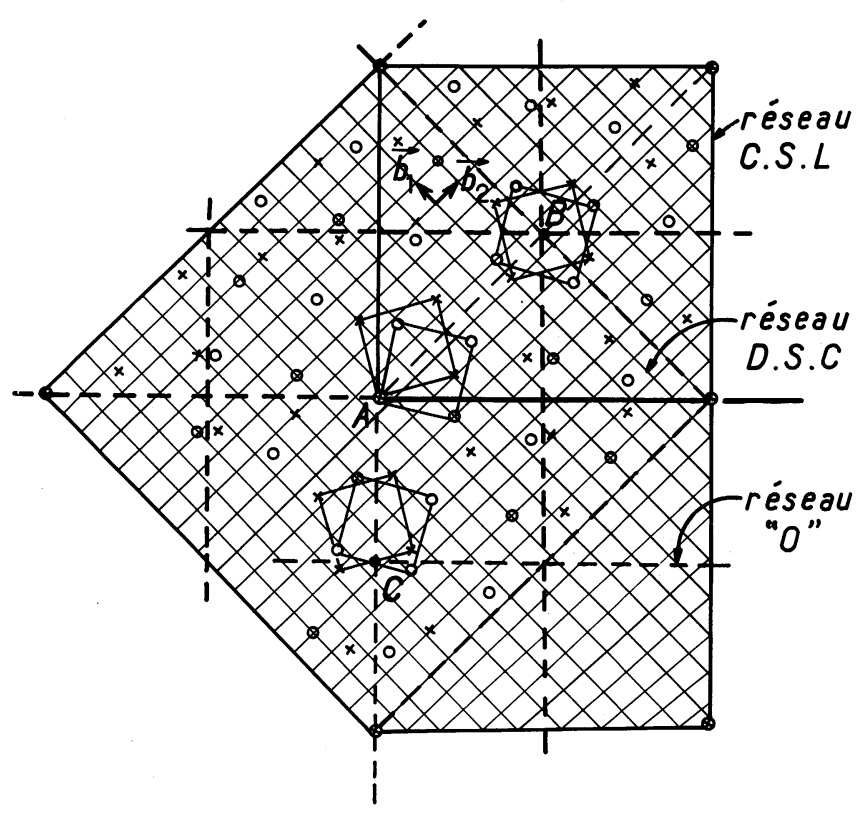

Fig. 1. - Vue des plans (001) de deux réseaux cubique simple interpénétrés, ayant subi une rotation l'un par rapport à l'autre de $\theta=28^{\circ} 1$ autour de [001] $(\Sigma=17)$; Construction du réseau de coïncidence $(-)$, du réseau « $O »(---)$, du réseau D.S.C. (-). Les points $\mathrm{A}, \mathrm{B}, \mathrm{C}$ du réseau « $\mathrm{O}$ » sont bien origines de la rotation reliant les deux cristaux. Les vecteurs $\mathbf{b}_{1}$ et $\mathbf{b}_{2}$ sont des vecteurs de base du réseau D.S.C.

[(001) planes of two interpenetrating simple cubic lattices rotated with respect to one another around [001] by $\theta=28^{\circ} 1(\Sigma=17)$; Construction of Coincidence Site Lattice (-), O-Lattice (- - - ) and D.S.C. Lattice; O-Lattice points A, B, C may be used as an origin for the rotational transformation of one lattice into the other; $\mathbf{b}_{1}$ and $\mathbf{b}_{2}$ are basis vectors of the D.S.C. Lattice.]
2.1 RECHERCHE D'UN INVARIANT. FORMALISME GÉOMÉTRIQUE. - 2.1.1 Le réseau de coincidence. Considérons le cristal A fixe et translatons le cristal B, de même structure, de telle façon que le plus grand nombre possible d'atomes des deux cristaux coïncident, les points de coïncidence forment alors un réseau périodique appelé «C.S.L." (coïncidence site lattice) (Fig. 1). Le degré de coïncidence est défini par $\Sigma$ (nombre de coïncidence ou indice de macle) qui est le rapport du volume de la maille de coincidence au volume de la maille élémentaire du cristal A ou B ; $\Sigma$ est aussi l'inverse de la fraction d'atomes du réseau associés à des sites de coïncidence. Le joint sera considéré comme coupant le biréseau selon un plan contenant une grande densité de sites communs. Le désordre du joint est d'autant plus grand que $\Sigma$ est élevé.

2.1.2 Le réseau « $\mathrm{O}$ ». - Bollmann [1, 2] considérant que les cristaux ne sont pas formés seulement des nœuds où se localisent les atomes mais également des interstices tente de trouver un invariant qui tienne compte de tout l'espace. Cela le conduit à définir un réseau de coïncidence généralisé où des points internes aux mailles élémentaires des deux grains, de mêmes coordonnées, se superposent par translation. On obtient alors un réseau de points de bon raccordement plus complet que le réseau «C.S.L. " : c'est le réseau « $\mathrm{O} »$; son appellation provient du fait que chaque point de ce réseau peut être considéré comme origine de la transformation qui relie le réseau $A$ au réseau $B$ (Fig. 1) $\left(^{2}\right)$.

Les sites du réseau C.S.L. sont donc des points particuliers du réseau « $O$ »; si cristallographiquement le réseau C.S.L. est une surstructure du réseau " $O$ », mathématiquement il en est un sous-ensemble. Il faut noter que, contrairement au réseau de coïncidence, le réseau " $\mathrm{O}$ » est une fonction continue de la transformation $\mathrm{A} \rightleftharpoons \mathrm{B}$.

2.1.3 Le réseau D.S.C. (Displacement Shift Complete). - Alors que le réseau « $O$ » préserve le motif cristallin tant du point de vue structure que position, le réseau D.S.C. définit tous les vecteurs déplacements qui permettent de passer du cristal B au cristal A en conservant seulement la structure du motif cristallin. Ce réseau peut être construit à partir de l'ensemble des vecteurs différence entre les deux réseaux des grains A et B en position de coïncidence (Fig. 1); ceux-ci ainsi que le réseau C.S.L. constituent donc des sous-ensembles du réseau D.S.C. Les méthodes analytiques pour déterminer le réseau D.S.C. sont décrites par Bollmann [1, 2] et Smith et Pond [3]. Il est important de dégager quelques propriétés fondamentales de ce réseau :

- L'unité de volume du réseau D.S.C. est proportionnelle à $1 / \Sigma$, ce qui implique que lorsque $\Sigma$ augmente, les vecteurs de base du réseau D.S.C. dimi-

$\left({ }^{2}\right)$ Pour le formalisme mathématique, voir la référence [1]. 
nuent. Dans le cas du système cubique simple, le réseau D.S.C. est le réseau réciproque du C.S.L.

- La connaissance de $\Sigma$ n'est pas suffisante pour décrire le réseau D.S.C. ; par exemple, dans le cas des métaux cubiques où une interface peut être décrite par une simple opération de rotation et par la nature cristallographique de la paroi (plan du joint), la connaissance de l'axe de rotation $R$ permet de définir les vecteurs de base du réseau D.S.C. :

- les vecteurs $\mathbf{b}_{1}$ et $\mathbf{b}_{2}$ sont perpendiculaires à $R$ - le vecteur $\mathbf{b}_{3}$ est sensiblement parallèle à $R$; lorsque $\Sigma \rightarrow \infty, \mathbf{b}_{1}$ et $\mathbf{b}_{2}$ deviennent infiniment petits, $\mathbf{b}_{3}$ conserve une longueur voisine d'une distance interatomique. Notons de suite que la position de l'axe de rotation par rapport au plan du joint (joint de torsion ou de flexion) entraîne que soit $\mathbf{b}_{1}$ et $\mathbf{b}_{2}$, soit $\mathbf{b}_{3}$ (ou des composantes importantes de ces vecteurs) sont contenus dans le plan du joint.

- Le réseau D.S.C. définit tous les vecteurs de Burgers possibles des dislocations parfaites du joint à l'équilibre.

2.2 LES DÉFAUTS LINÉAIRES DU JOINT DE GRAINS. Le joint de grains peut contenir, comme le grain luimême, des défauts ponctuels, linéaires et plans. L'étude des lacunes et des interstitiels semble mieux abordée par les modèles analytiques $[4,5,6,7]$ ou par le modèle d'empilement de polyèdres $[8,9](\S 5)$. Par ailleurs, . la description des fautes d'empilement auxquelles sont associées des dislocations partielles [10] reste très complexe. C'est pourquoi, nous décrivons ici essentiellement les défauts linéaires pour lesquels le formalisme de Bollmann est spécialement bien adapté [11 à 18].

2.2.1 Les dislocations « intrinsèques 》 ou «structurales ». - Pour préserver les invariants décrits précédemment et ainsi minimiser l'énergie de configuration, il est nécessaire de faire intervenir dans la structure d'équilibre du joint des relaxations atomiques qui accommodent la désorientation réelle des deux cristaux :

- d'une part, par rapport à l'invariant monocristallin : ce sont les dislocations primaires dont les vecteurs de Burgers sont ceux de la matrice ;

- d'autre part, par rapport à l'invariant que constitue toute position de coïncidence : ce sont les dislocations secondaires dont les vecteurs de Burgers sont ceux du réseau D.S.C.

Une description géométrique de ces dislocations et de leurs circuits de Burgers associés (appelés circuits de Read) est donnée par Hirth et Balluffi [19].

a) Les dislocations primaires. - Dans le cas des joints à faible désorientation, il est relativement aisé de concevoir que le plan du joint passe autant que possible par les sites du réseau « $\mathrm{O} »$; les zones de mauvais raccordement des deux cristaux $A$ et $B$ situées entre les sites « $O$ » constituent les cœurs d'un réseau de dislocations primaires qui rendent compte

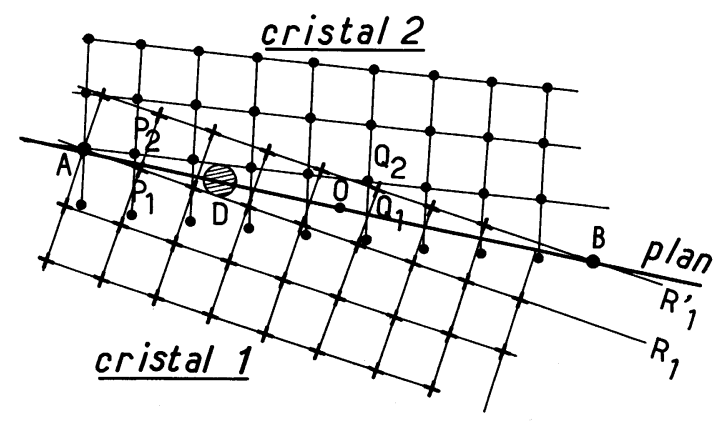

Fig. 2. - Définition des dislocations primaires grâce au réseau « $\mathrm{O}$ »:

$\mathrm{A}, \mathrm{B}, \mathrm{O}$ sites du réseau « $\mathrm{O}$ »

$\left.\begin{array}{l}Q_{1} \text { et } Q_{2} \\ P_{1} \text { et } P_{2}\end{array}\right\}$ points homologues assimilés à un seul site

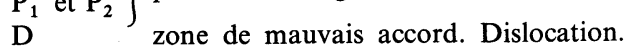

[View of primary dislocations using the O-lattice :

A, B, O O-lattice sites

$\left.\begin{array}{l}Q_{1} \text { and } Q_{2} \\ P_{1} \text { and } P_{2}\end{array}\right\}$ points so closed that they can be seen as one site

$\mathrm{D}$ point of bad matching. Dislocation.]

totalement de la désorientation entre les grains (Fig. 2) [20].

L'arrangement régulier de ces dislocations dans le plan du joint peut être observé en microscopie électronique grâce au contraste de contrainte (Fig. 3) [21] ; la distance entre les lignes de dislocations est, dans le cas d'un joint de torsion [001] :

$$
d=\frac{|\mathbf{b}|_{\text {matrice }}}{2 \sin \theta / 2}
$$

où b est un vecteur de Burgers du monocristal et $\theta$ l'angle de désorientation entre les cristaux voisins. Cette distance correspond évidemment au vecteur de base du réseau « $\mathrm{O}$ ». Lorsque la désorientation augmente, la distance entre les dislocations diminue,

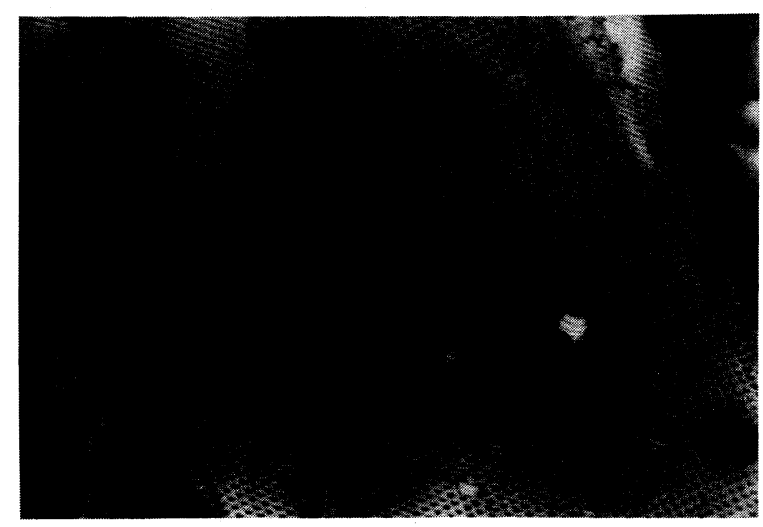

Fig. 3. - Mise en évidence dans l'or d'un réseau bidimensionnel de dislocations primaires alignées selon les directions $\langle 110\rangle$ dans un joint de torsion [001] $\left(\theta \# 3^{\circ}, \Sigma=1, d=50 \AA\right.$ [91]).

[Square networks of screw primary dislocations in gold, in [001] twist boundary $\left(\theta \# 3^{\circ}, \Sigma=1, d=50 \AA\right)$; the dislocations line are parallel to $\langle 110\rangle$ directions [91].] 
leurs champs de contrainte se superposent de telle façon que chaque dislocation n'a plus de signification physique. Ce fait est confirmé, dans le cas de l'or par exemple, par l'impossibilité de détecter, par un contraste de déformation, le réseau de relaxations primaires associé à un joint de torsion [001] lorsque l'angle de désorientation atteint $15^{\circ}$.

Cependant, le réseau " $O$ " étant une fonction continue de la transformation qui relie le cristal $\mathrm{A}$ au cristal B, formellement c'est-à-dire d'un point de vue géométrique, les dislocations primaires doivent exister. Cette continuité de la description du joint paraît d'ailleurs confirmée par les résultats récents de Sass, Tan et Balluffi $[22,23]$ basés sur le fait que le réseau de relaxations dans le plan du joint constitue une grille optique donnant lieu à des taches supplémentaires sur les clichés de diffraction des électrons et des rayons $X$ (cf. $\S 4.2 .2$ ) ; ces taches peuvent être utilisées pour obtenir une image du réseau en microscopie électronique et la distance des rangées satisfait bien à la relation (1) (Fig. 4). En s'appuyant sur une propriété fondamentale du réseau " $\mathrm{O}$ », on peut donc dire formellement qu'une dislocation primaire est associée à tout vecteur de translation qui conserve la structure et la position du motif cristallin de part et d'autre du joint.

La distinction entre la signification physique et la signification géométrique des dislocations primaires demeure néanmoins comme le soulignent diverses limitations de l'application du réseau " $\mathrm{O}$ » au modèle du joint $[10,24,25,26]$. Mais il ne faut pas perdre de vue que le formalisme de Bollmann ne prétend pas décrire la réalité du joint de grain mais constitue une approche possible pour sa connaissance.

b) Les dislocations secondaires. - Lorsque les deux cristaux A et B sont fortement désorientés, de nom-

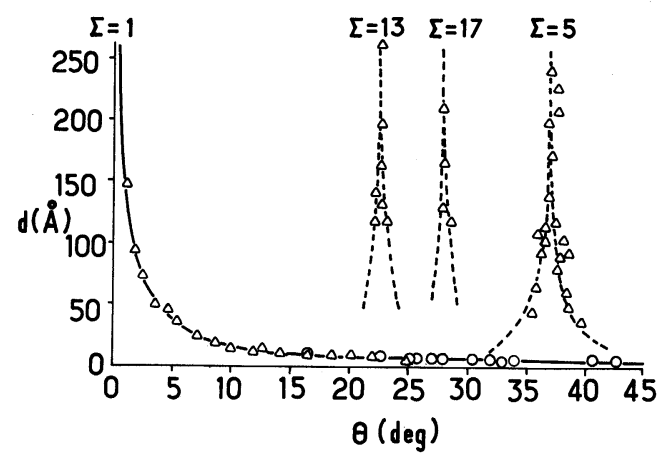

Fig. 4. - Distance des dislocations primaires et secondaires observées par microscopie électronique dans l'or en fonction de l'angle de torsion autour d'un axe [001] : $\triangle$ : observation sur l'image; $\bigcirc:$ observation par diffraction; - $-: d=\frac{a \sqrt{2}}{2 \sin \theta / 2} ; \cdots$ : $d=\frac{b_{\text {D.s.C. }}}{2 \sin \Delta \theta / 2}$

[Observed primary and secondary relaxation spacing in [001] Twist boundaries in gold as a function of twist angle $\theta: \triangle$ : observed by microscopy; $\bigcirc:$ observed by diffraction; $-d=\frac{a \sqrt{2}}{2 \sin \theta / 2}$; $\ldots: d=\frac{b_{\text {D.s.c. }}}{2 \sin \Delta \theta / 2}$. $]$ breux auteurs ont souligné le fait que le joint tend à préserver autant que possible la structure correspondant au réseau de coïncidence le plus proche [27, 28]. L'écart angulaire $\Delta \theta$ entre la position réelle et la position de coïncidence est alors compensé par un réseau de dislocations secondaires [29] dont les vecteurs de Burgers sont ceux du réseau D.S.C. et dont l'espacement, dans le cas d'un joint de torsion [001], par exemple, est :

$$
d=\frac{|\mathbf{b}|_{\text {D.s.C. }}}{2 \sin \Delta \theta / 2} .
$$

De tels réseaux peuvent être calculés et construits par analogie aux réseaux de dislocations primaires en introduisant mathématiquement la notion de réseau " $\mathrm{O}_{2}$ " [30]. L'appellation secondaire provient du fait que ces dislocations peuvent être considérées comme des défauts liés à une irrégularité dans la périodicité des dislocations primaires.

Il convient à nouveau de distinguer entre l'aspect formel et la réalité physique de ces relaxations : si on considère la formule (2), on constate que

- si $\mathbf{b}_{\text {D.s.c. }}$ diminue, c'est-à-dire si $\Sigma$ augmente, la distance entre les dislocations secondaires décroît, ce qui équivaut à une répartition plus ou moins uniforme des contraintes. Notons par ailleurs que le contraste associé à une dislocation de petit vecteur de Burgers est faible. Ces remarques expliquent, sans doute, l'absence du point de vue expérimental, de dislocations secondaires dans les joints de faible désorientation $\left(\theta<16^{\circ}\right)$ correspondant à des $\Sigma$ élevés bien qu'il soit difficile de concevoir que le réseau de dislocations primaires ne contienne aucune perturbation;

- si $\Delta \theta$ augmente, pour un même nombre de coïncidence, la distance entre dislocations diminue également. Bien qu'il soit toujours possible de concevoir une hiérarchie dans les vecteurs de Burgers du réseau D.S.C. qui permettrait d'augmenter l'écart angulaire [31], expérimentalement il existe une limite au-delà de laquelle on n'observe plus de dislocations secondaires (Fig. 4) [32]. Cette limite est d'autant plus petite que $\Sigma$ est grand, ce qui explique que seuls, certains réseaux de coïncidence $(\Sigma<25)$ peuvent être utilisés et qu'un joint de désorientation intermédiaire entre deux positions de haute coïncidence n'a pas actuellement d'interprétation expérimentale.

Donc si le formalisme de Bollmann permet théoriquement de décrire tout joint en termes de dislocations primaires et secondaires, la réalité physique de celles-ci est limitée par la résolution des techniques actuelles d'observation. Cette réalité dépend :

- du contraste associé aux dislocations, fonction, entre autres, de leur vecteur de Burgers donc de $\Sigma$;

- de la distance entre dislocations fonction de $|\mathbf{b}|$ et de $\Delta \theta$ (dans le cas où $\Sigma=1, \theta=\Delta \theta$ et les formules (1) et (2) sont équivalentes). 
Lorsque la distance diminue, on peut considérer que les contraintes sont réparties uniformément dans le joint [33] ou, ce qui est physiquement équivalent, que les cœurs des dislocations parfaites se chevauchent [31].

En conclusion, si le modèle de Bollmann semble géométriquement ne pas avoir de limite, ce qui est déterminant en dernier lieu c'est la réalité physique des dislocations intrinsèques du joint de grains.

2.2.2 Les dislocations extrinsèques. - Alors que les dislocations intrinsèques font partie de la structure d'équilibre du joint de grain $\left({ }^{3}\right)$, qu'elles sont distribuées périodiquement imposant l'absence de contraintes à longue distance, les dislocations extrinsèques se surimposent à la structure d'équilibre, elles sont réparties au hasard et leurs champs de contrainte ne s'annulent pas à grande distance. Leur origine provient essentiellement de l'interaction des dislocations de la matrice avec le joint et leur étude consiste donc, tout d'abord, à déterminer si cette interaction se fait ou non avec dissociation. Les diverses réponses à cette question restent l'objet de controverses dans la mesure où il n'existe pas de méthode expérimentale véritablement bien établie pour déterminer les vecteurs de Burgers des dislocations extrinsèques $[34,35,36]$ (cf. $\S 4.1)$. Nous allons donc évoquer les différents résultats :

- Certains travaux mettent en évidence le fait que la dislocation de réseau conserve son vecteur de Burgers en s'intégrant dans le joint de grain [37, 38]. Statistiquement, cela conduit à une forte probabilité d'existence d'une composante coin de la dislocation extrinsèque rendant compte de sa métastabilité dans le plan du joint et lui conférant, à température plus élevée, un rôle important dans les processus d'interaction avec les défauts ponctuels.

- Ces mêmes propriétés dues à la possibilité de montée de la dislocation se retrouvent dans les résultats de Schober et Balluffi sur les joints à faible désorientation où il y a dissociation de la dislocation de matrice lors de son incorporation dans le joint mais les différents vecteurs de Burgers impliqués sont des vecteurs de Burgers du réseau [39]. Ces auteurs ]nterprètent la direction de la dislocation extrinsèque en utilisant la procédure de Frank [40] et montrent que son contraste s'explique par l'existence d'un vecteur de Burgers effectif perpendiculaire au plan du joint (cf. § 3.3.1).

- Dans le cas des joints à forte désorientation proche de la coïncidence, de nombreux résultats vont à l'appui de la dissociation du vecteur de Burgers de la matrice en vecteurs de Burgers du réseau D.S.C. en respectant la règle de Frank :

$$
\mathbf{b}_{\text {matrice }}=\sum_{n} \mathbf{b}_{n \text { (D.S.C.) }} \text {. }
$$

${ }^{3}$ ) La somme de leurs vecteurs de Burgers est égale au vecteur différence de fermeture du circuit de Frank rendant compte totalement de la désorientation entre les cristaux [13]
Cette réaction est favorisée car $\left|\mathbf{b}_{\text {D.s.c. }}\right|$ est inférieur à $\left|\mathbf{b}_{\text {matrice }}\right|$ et il existe donc une force motrice pour la dissociation, mais celle-ci n'est observable que si les vecteurs de base du réseau D.S.C. sont suffisamment grands. Dans cette limite, des réactions de dissociation conduisant, au moins, à la mise en évidence d'un vecteur du réseau D.S.C. ont pu être déterminées dans de nombreux joints de torsion et de flexion [41 à 48], et même dans le cas de joints plus proches des joints réels auxquels s'applique le modèle du Plane Matching (cf. § 3.2) [49]. La dissociation à température ambiante est plus ou moins rapide selon que les vecteurs de Burgers sont parallèles au plan du joint et que les composantes sont glissiles ou qu'il existe une composante perpendiculaire au plan du joint, ce qui semble être le cas le plus général ; il peut y avoir annihilation des composantes dans le plan du joint et la dislocation a alors un caractère purement coin.

- Plus récemment, des réactions de dissociation plus complexe ont été envisagées avec intervention de dislocations partielles du joint de grain $[10,50]$. Ces réactions sont difficiles à étudier car, dans le joint contrairement au grain, il n'existe pas de relation simple entre les vecteurs de Burgers des dislocations parfaites et ceux des dislocations imparfaites.

Tous les résultats évoqués précédemment ont en commun la possibilité d'existence d'une composante coin de la dislocation extrinsèque lui conférant un rôle important dans certaines propriétés des interfaces (déformation plastique, migration, ségrégation...).

Une autre catégorie de résultats, le plus souvent sur des joints à forte désorientation mal caractérisés cristallographiquement, évoquent une distribution uniforme dans le joint des contraintes liées aux dislocations de matrice lors de leur incorporation [51, 52, $53,54]$. Mais, dans ce cas, on est amené à un dilemme déjà rencontré dans l'étude des dislocations intrinsèques : s'agit-il réellement d'une dispersion ou d'une dissociation en un nombre discret de dislocations possédant des vecteurs de Burgers trop petits pour être détectables [49] ?

On peut avancer que les preuves d'existence des dislocations extrinsèques du joint comme résultat de l'interaction des dislocations de matrice avec les dislocations intrinsèques semblent prépondérantes même si les réactions de ce processus sont encore mal définies expérimentalement. Certaines observations communes aux différentes études peuvent être dégagées :

- Les dislocations extrinsèques ne se terminent jamais à l'intérieur du joint de grain ; elles se referment sur elles-mêmes, se terminent à la limite du joint ou se raccordent à d'autres dislocations, en particulier, aux dislocations de matrice (Fig. 5).

- Dans les régions libres de dislocations extrinsèques, le réseau de dislocations intrinsèques n'est pas affecté (Fig. 6).

- Ce réseau est perturbé dans les régions balayées par la montée des dislocations extrinsèques ou lorsque 


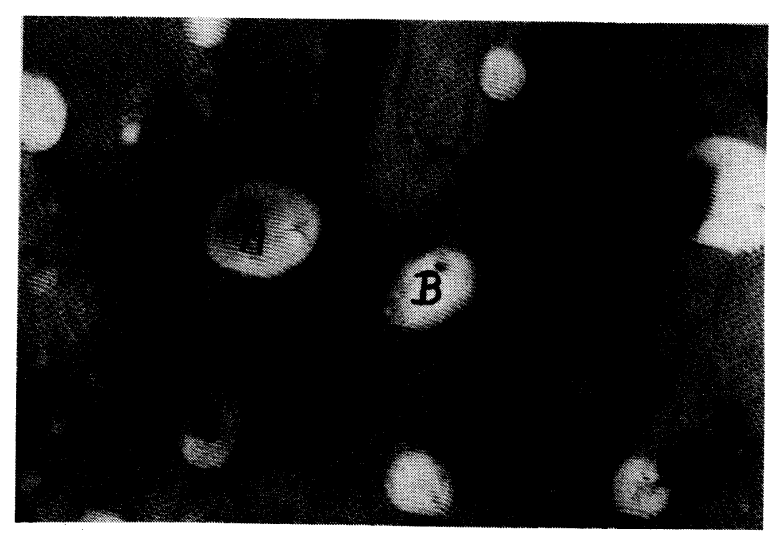

Fig. 5. - Observation de dislocations extrinsèques dans un joint de torsion [001] de l'or. Dans les régions telles que A et B, le bicristal n'est pas soudé, on note que les dislocations extrinsèques se terminent à la limite du joint [91].

[Observed extrinsic dislocations in [001] twist boundary in gold. In some regions such as $\mathrm{A}$ and $\mathrm{B}$, the two crystals are not good welded, one can see that the E.G.B.D's end to the grain boundary limit [91].]

plusieurs dislocations de matrice très rapprochées ont interagi avec le joint (Fig. 6).

Les deux dernières remarques signifient, qu'en général, la désorientation entre les grains n'est pas globalement modifiée par la présence de dislocations hors d'équilibre. Cependant, dans certaines conditions, il n'est pas exclu que les dislocations de la matrice s'incorporent totalement dans la structure du joint, c'est-à-dire que leur dissociation conduise à des dislocations parfaites arrangées périodiquement, modifiant ainsi la désorientation globale des deux cristaux : ces dislocations prennent alors un caractère intrinsèque. Il y a donc une ambiguïté terminologique à définir une dislocation extrinsèque uniquement comme une dislocation de réseau «dans le joint»,

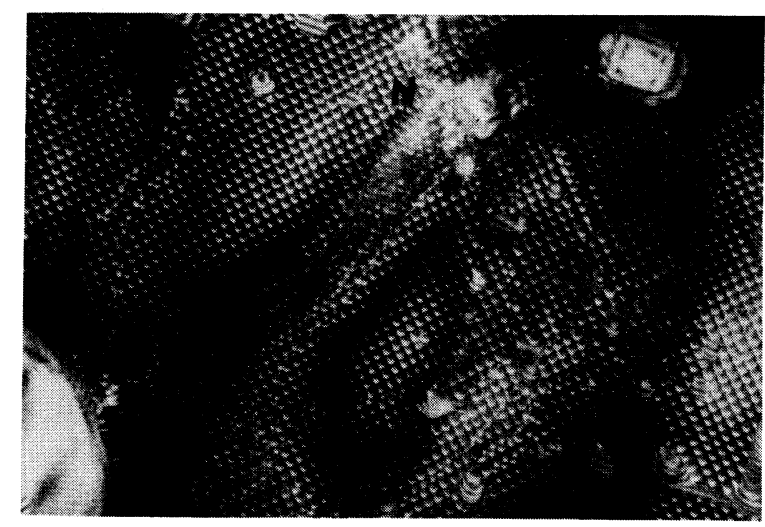

Fig. 6. - Dans la région MNPQ d'un joint de torsion [001], la structure intrinsèque est affectée par l'accumulation de dislocations extrinsèques [91].

[In the MNPQ area of a twist [001] grain boundary $\left(\theta \# 3^{\circ}\right)$ the intrinsic network is disrupted by impinging several lattice dislocations [91].] il faut tenir compte du résultat de l'interaction matrice-joint de grain, en particulier, du caractère isolé de la dislocation extrinsèque [55].

Notons également qu'une dislocation extrinsèque parfaite peut être primaire ou secondaire selon son vecteur de Burgers.

Enfin, nous tenons à souligner ici un exemple caractéristique du parallélisme évoqué brièvement au début de cet article entre l'étude des défauts du cristal et l'étude des défauts du joint de grains : ainsi, une dislocation de matrice détruit localement la périodicité cristalline, ce qui peut se traduire graphiquement par l'existence d'un plan supplémentaire et de régions en tension et compression de part et d'autre du défaut, de la même façon, une dislocation extrinsèque détruit la périodicité de la structure intrinsèque du joint, elle peut être révélée graphiquement par une perturbation du réseau D.S.C. [15-50] considéré comme rigide dans le formalisme de Bollmann (Fig. 7).

2.2.3 Les ledges ou marches. - Une grande confusion existe dans la littérature concernant ce type de défauts le plus souvent associé à une dislocation extrinsèque sous forme de marche à l'échelle atomique ( $h \simeq 3 \AA$ ) $[19,56]$. Certains travaux font plus ou moins l'analogie entre dislocations et ledges quelle que soit la hauteur de la marche dans le plan du joint $[57,58$, $59,60]$, d'autres utilisent la terminologie de steps pour des marches pouvant atteindre $25 \AA$ [61], mais il convient alors de les considérer comme des défauts plans.

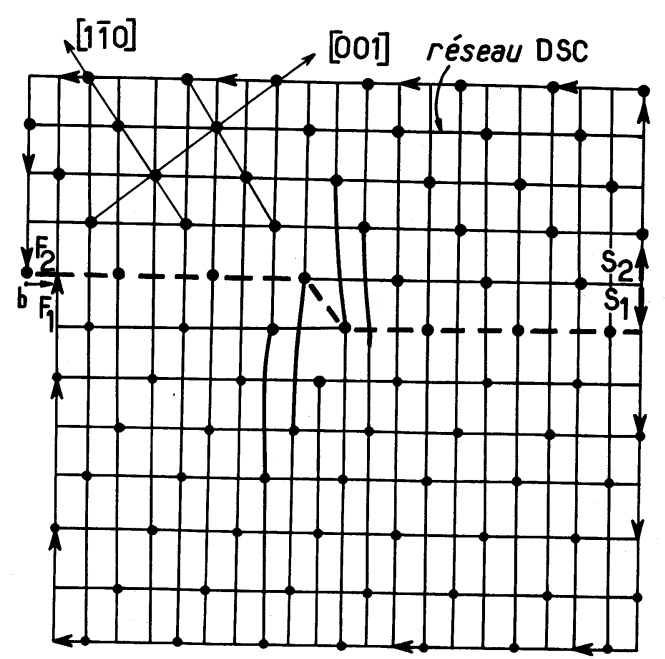

Fig. 7. - Mise en évidence par construction d'un circuit de Frank dans le réseau D.S.C. d'une dislocation extrinsèque du type Shockley $(\mathbf{b}=1 / 6\langle 112\rangle)[19]$.

[Diagram showing an extrinsic dislocation (of Shockley type) in the D.S.C. lattice by mean of a Frank circuit, $b=1 / 6<112>$ [19].]

Une marche pure, sans contrainte associée (sans caractère de dislocation) doit avoir son plan composé entièrement de sites de coïncidence (Fig. 8) [62]. 


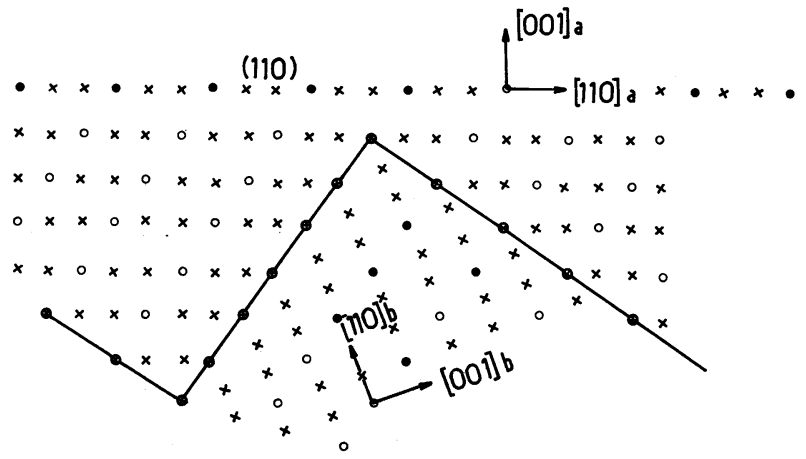

Fig. 8. - Diagramme montrant une marche (ou ledge) libre de contrainte dans un joint de macle $\Sigma=3$. Le plan du joint est composé entièrement de sites de coïncidence [15].

[Diagram showing a strain free ledge in a $\Sigma=3$ twin boundary. The grain boundary plane is entirely composed of C.S.L. sites [15].]

Une telle marche se rencontre rarement, cependant on peut concevoir son existence dans trois cas :

- Lors de la préparation de lames minces bicristallines, par soudage de deux films qui présentent en regard des marches s'épousant parfaitement; ces marches résultent elles-mêmes de l'évaporation sur des monocristaux de sel imparfaitement plans.

- Par existence sur la surface libre limite de chaque monocristal avec le joint de grain d'un profil en créneau comme résultat de l'équilibre thermodynamique et par analogie avec une surface libre qui n'est parfaitement plane qu'au zéro degré absolu.

- Lors du passage d'une dislocation d'un grain à l'autre sans contrainte résiduelle dans l'interface c'est-à-dire si les plans de glissement des deux cristaux coïncident sensiblement.

Il faut noter qu'une telle marche n'a pas un caractère hors d'équilibre mais qu'elle peut contenir des dislocations intrinsèques [19]. En pratique, deux cas peuvent se rencontrer :

- La marche pure est nettement visible, sa largeur apparente en microscopie électronique est supérieure à celle d'une dislocation extrinsèque et il n'existe pas de condition d'extinction. Le critère souvent retenu pour caractériser cette marche, à savoir le déplacement local des franges d'épaisseur du joint, doit être utilisé avec précaution car un même déplacement peut résulter d'un changement de $\mathbf{s}$ (écart à la position de Bragg) dû, par exemple, à une modification d'épaisseur du cristal diffractant [62].

- La marche n'est pas visible, elle existe comme résultat de l'émergence d'une dislocation extrinsèque $\mathrm{du}$ joint dans l'un des grains adjacents $(h \#|\mathbf{b}|)$ par analogie avec ce qui se passe à la surface libre du matériau lorsque débouche une dislocation de matrice.

3. Application du modèle géométrique à différentes structures de joints de grains. - Nous avons vu dans le chapitre précédent qu'il convient de toujours distinguer entre la réalité géométrique et la réalité physique de l'approche de Bollmann. Sur la base de cette dichotomie, les joints de grains peuvent être classés globalement en deux groupes :

- Les joints spéciaux pour lesquels on peut déterminer expérimentalement un ordre c'est-à-dire qu'ils possèdent une certaine cristallinité ; ce sont les joints de faible désorientation $\left(\theta<15^{\circ}\right)$ et les joints à grand angle proches de la coïncidence.

- Les joints généraux dans lesquels aucune périodicité ne peut être révélée compte tenu des techniques actuelles, ce qui n'exclut pas un certain degré d'organisation $[44,63,64]$.

Il ressort très nettement que cette classification ne peut être rigoureuse et aussi qu'elle est évolutive. Elle dépend, entre autres, du progrès des techniques à détecter un ordre et dialectiquement du concept d'ordre. On peut dire qu'à tout moment de la connaissance, il existe des joints plus ou moins spéciaux comme on le verra avec le modèle du Plane Matching. C'est pourquoi l'objectif de ce chapitre n'est pas de rendre compte de toutes les applications du modèle géométrique à différents types de joints et dans différents matériaux, ce qui ne saurait d'ailleurs être exhaustif, mais plutôt de cerner, sur quelques exemples, la réalité physique de l'approche géométrique basée sur le concept de coïncidence. Ceci nous conduit à construire ce chapitre sur quatre idées :

a) Dégager les limites de l'application du modèle de Bollmann dans le cas des joints très spéciaux de torsion et de flexion autour d'axes de faibles indices;

b) Montrer, au contraire, son extension possible aux joints décrits par le modèle du Plane Matching, joints qui peuvent être considérés comme intermédiaires entre les joints spéciaux précédents et les joints généraux ;

c) Rendre compte de certaines réactions de dissociation des dislocations de la matrice en dislocations extrinsèques du joint possédant ou non des vecteurs de Burgers du réseau D.S.C. ;

d) Tenter de voir dans quelle proportion un polycristal présente des interfaces auxquelles s'applique plus ou moins le modèle géométrique, c'est-àdire, faire le point sur les joints dits au hasard, terminologie qui englobe tous les types de joints : joints à faible désorientation, joints à forte désorientation spéciaux et généraux.

3.1 JoINTS SPÉCIAUX C.S.L. - Ces joints sont observés, en général, sur des bicristaux fabriqués selon différentes techniques :

- par évaporation de films du métal sur des supports monocristallins préorientés suivie de soudure de deux de ces films [65];

- par solidification lente à partir de germes préorientés (méthode de Czochralski) [66] ;

- plus récemment, par croissance épitaxiale sur bicristal de sel gemme [67]. 
Selon la technique de préparation, le plan du joint est, en moyenne, parallèle, perpendiculaire ou incliné par rapport à la surface de la lame mince. Nous avons fait choix de présenter, plus particulièrement, les résultats obtenus sur des joints parallèles au plan de surface du bicristal, cas où les contrastes observés sont, relativement, plus simples $\left({ }^{4}\right)$. Compte tenu de notre objectif, réalité et limite de l'ensemble du formalisme géométrique, il nous a semblé plus judicieux de scinder la présentation des résultats concernant les dislocations intrinsèques en applications aux joints de torsion puis aux joints de flexion et, non pas, comme c'est souvent le cas dans la littérature aux joints à faible puis à forte désorientation. En effet, le modèle théorique permet d'envisager l'existence de relaxations primaires et secondaires quel que soit l'angle de désorientation (ceci a été prouvé récemment pour les dislocations primaires $[22,23])$ mais les limites d'explication diffèreront toujours fortement selon que les réseaux observés sont constitués de dislocations vis ou de dislocations coin. La plus grande difficulté à rendre compte d'un joint de flexion a trois origines essentielles :

- la préparation de ces joints qui ne permet pas de contrôler rigoureusement la désorientation, mais ce facteur est améliorable;

- les conditions de contraste des dislocations coin dans le plan du joint sur lesquelles il est plus difficile d'influer;

- le fait que le plan du joint n'est pas, ou rarement, un plan de glissement, cause qui sera toujours déterminante.

3.1.1 Joints de torsion. - a) Dislocations primaires. - Considérons un joint de torsion autour d'un axe [001] pour lequel la continuité du réseau " $O$ " permet de prévoir, quelle que soit la désorientation, l'existence d'un réseau de dislocations vis parallèles aux directions $\langle 110\rangle$ dans le plan du joint et distantes de :

$$
d=\frac{\left|\mathbf{b}_{\text {matrice }}\right|}{2 \sin \theta / 2} \text {. }
$$

Dans le cas de l'or, par exemple,

$$
b=a / 2\langle 110\rangle=2,89 \AA[21] .
$$

Dans les joints faiblement désorientés, chaque dislocation appartenant au réseau est résolue individuellement par le contraste résultant de son champ de contrainte : on observe alors des réseaux plus ou moins réguliers selon l'angle de désorientation $\theta$ (Fig. 9). L'irrégularité constatée lorsque $\theta<2^{\circ}$

$\left.{ }^{4}\right)$ Une meilleure compréhension des résultats nécessite la connaissance des différents types de contraste des joints de grains observés en microscopie électronique (cf. § 4); il nous a semblé préférable de ne pas scinder les modèles décrits précédemment et leurs applications.

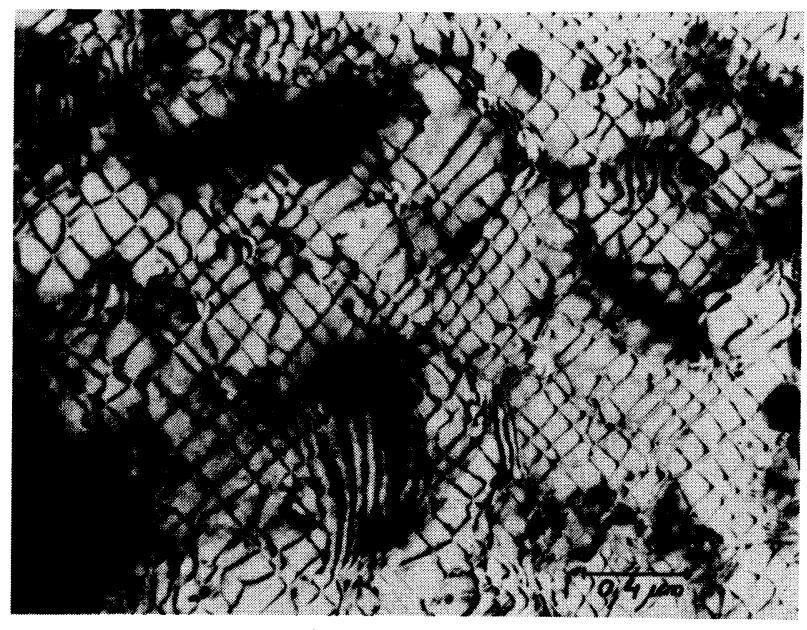

Fig. 9. - Dans un joint de torsion [001] de l'or faiblement désorienté $\left(\theta \# 1^{\circ} 5\right)$, le réseau de dislocations primaires est très irrégulier [91].

[In a low angle [001] twist boundary $\left(\theta \# 1^{\circ} 5\right)$ in gold, the network of primary dislocations is very irregular [91].]

s'explique, entre autres, en considérant la répercussion sur $d$ de l'erreur relative $\operatorname{sur} \theta$ :

Soit $\Delta \theta=0^{\circ} 5$,

$$
\begin{aligned}
& \left.\begin{array}{lll}
\text { si } & \theta=1^{\circ} & d=165 \AA \\
\text { si } & \theta=1^{\circ} 5 & d=110 \AA
\end{array}\right\} d=55 \AA
\end{aligned}
$$

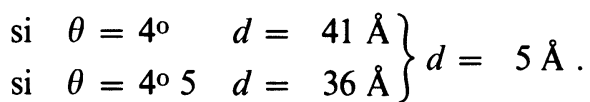

Il est évident que, dans le premier cas, l'erreur sur $d$ sera très visible en microscopie électronique alors que, vers $\theta=4^{\circ}$, les lignes de dislocations apparaîtront continues.

On a déjà vu (cf. § 2.2) que lorsque $d$ diminue, les champs de contrainte des dislocations se superposent et leur contraste individuel n'est plus résolu en microscopie électronique. Bien que la limite théorique d'observation du contraste de contrainte avait été déterminée par A. R. Thölen [68], de l'ordre de $54 \AA$ pour l'or, l'expérience prouve que ce contraste opère jusqu'à $d=16 \AA$, ce qui correspond à $\theta \simeq 10^{\circ}[22,23]$. De plus, la réalité des dislocations primaires a été étendue à toute désorientation autour de [001] par Sass et ses collaborateurs $[22,23,69,70]$ en considérant le réseau de relaxations comme une grille optique donnant lieu à des taches supplémentaires sur les clichés de diffraction des rayons $\mathrm{X}$ et des électrons. Ces taches sont disposées en carré autour des taches de la matrice, à une distance de celles-ci égale à l'inverse de la distance des dislocations dans le plan du joint et selon des directions parallèles à la grille (Fig. 10) (cf. § 4.2.2). L'analyse des clichés de diffraction a permis ainsi de déterminer indirectement l'existence de dislocations primaires jusqu'à $42^{\circ} 5$ de désorientation ; mais la visualisation de ces réseaux (image du réseau " $O »$ ) n'a été possible que pour 

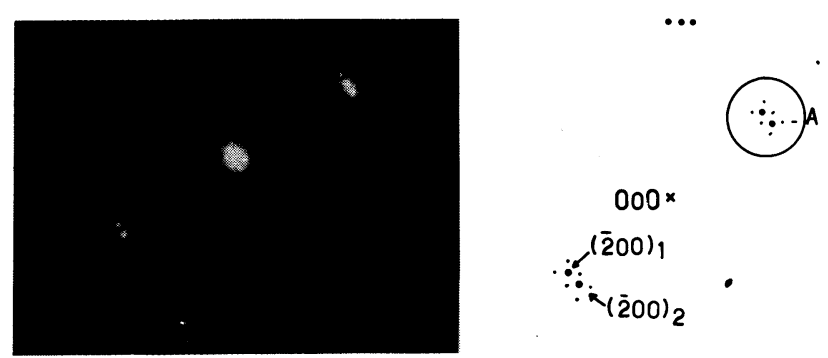

a

-

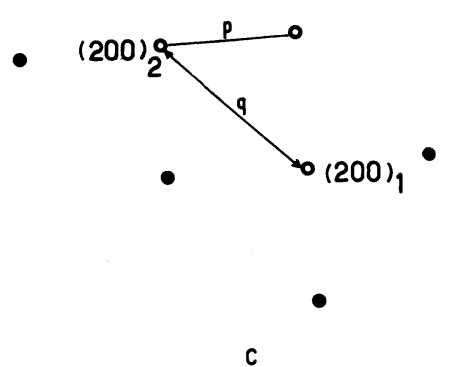

Fig. 10. - a) Diagramme de diffraction caractéristique d'un joint présentant un réseau de dislocations primaires. $b$ ) Interprétation. c) Agrandissement de la région $A$ : $O$ : réflexions de la matrice; - : réflexions dues à la grille optique que constitue le joint.

[a) Electron Diffraction pattern from a specimen containing a boundary with a square network of primary dislocations. $b$ ) Interpretation. c) Enlarged A region : $\bigcirc$ : crystals diffraction spots ; : extra-spots due to the grain boundary scattering.]

$\theta<25^{\circ}$, ce qui correspond, pour l'or, à $d \simeq 7 \AA$ (Fig. 4) [23]. Cette limite est vraisemblablement due au fait que le contraste devient très faible puisque la résolution du microscope devrait permettre d'atteindre $2 \AA$.

Les résultats concernant les dislocations primaires dans les joints de torsion affirment l'utilité du réseau « $\mathrm{O}$ », fonction continue de la désorientation entre grains, comme base de description des joints de grains.

b) Dislocations secondaires. - Les prévisions théoriques de ces dislocations découlent de la construction du réseau D.S.C. [71, 72]. Elles sont résumées et comparées aux résultats expérimentaux dans le cas de l'or et pour des joints de torsion [001] dans le tableau I [11].

Tableau I. - Réalité physique des dislocations secondaires dans des joints de torsion [001] de l'or.

\begin{tabular}{rcccc}
\multicolumn{5}{c}{$\begin{array}{c}\text { Vecteurs } \mathbf{b}_{1} \\
\text { et } \mathbf{b}_{2} \text { du réseau }\end{array}$} \\
$\Sigma$ & $\theta$ & D.S.C. & $b(\AA)$ & Observation \\
- & - & - & - & - \\
5 & $36^{\circ} 9$ & a $/ 10\langle 310\rangle$ & 1,29 & oui \\
13 & $22^{\circ} 6$ & $\mathrm{a} / 26\langle 510\rangle$ & 0,80 & oui \\
17 & $28^{\circ} 1$ & $\mathrm{a} / 34\langle 530\rangle$ & 0,70 & oui \\
25 & $16^{\circ} 3$ & $\mathrm{a} / 50\langle 710\rangle$ & 0,58 & non
\end{tabular}
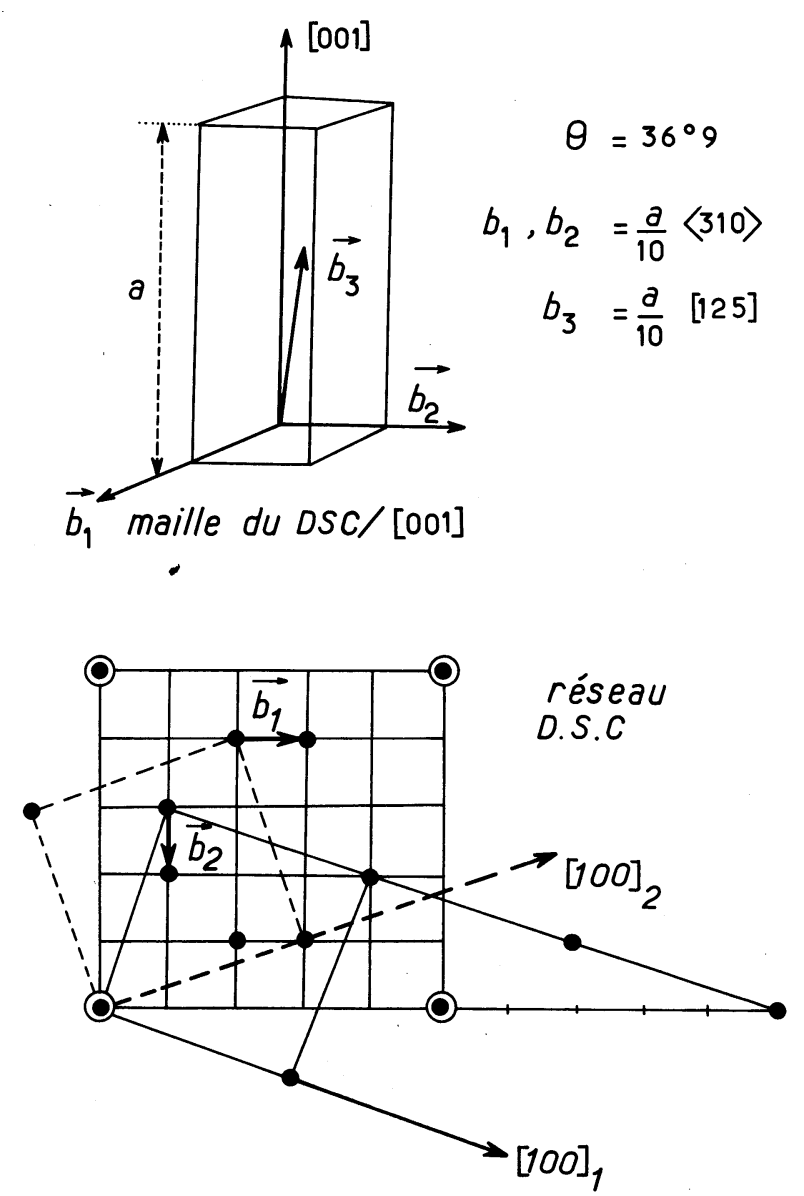

Fig. 11. - a) Maille du réseau D.S.C. dans un bicristal C.F.C $\Sigma=5, \theta=36^{\circ} 9$, axe de rotation [001]. b) Schéma montrant les vecteurs de Burgers $\mathbf{b}_{1}$ et $\mathbf{b}_{2}$ des dislocations secondaires dans un joint de torsion [001], $\Sigma=.5 ; \mathbf{b}_{1}$ et $\mathbf{b}_{2}$ sont parallèles aux directions $\langle 310\rangle$ des deux cristaux.

[a) D.S.C. unit in a F.C.C. bicrystal, $\Sigma=5, \theta=36^{\circ} 9$ rotation axis [001]. b) Diagram showing the Burgers vectors $\mathbf{b}_{1}$ and $\mathbf{b}_{2}$ of the secondary dislocations in a [001] twist boundary $(\Sigma=5)$; $\mathbf{b}_{1}$ and $\mathbf{b}_{2}$ are parallel to the $\langle 310\rangle$ directions of the two crystals.]

Précisons sur l'exemple $\Sigma=5$ la configuration du réseau D.S.C. et donc la détermination des vecteurs de Burgers (Fig. 11). Sur cette base, on peut prévoir l'existence d'un réseau de dislocations vis parallèles aux directions $\langle 310\rangle$ dans le plan du joint (le vecteur $\mathbf{b}_{3}$ ne peut donner lieu à des dislocations vis dans un joint de pure torsion puisqu'il est sensiblement perpendiculaire au plan du joint). C'est bien ce que l'expérience constate, du moins lorsque $\Delta \theta$ reste inférieur à $2^{\circ}$ environ, c'est-à-dire lorsque $\mathrm{d}$ est supérieur à $50 \AA$ [29]. Le fait que cette limite est bien supérieure à celle des dislocations primaires $(16 \AA)$ s'explique par une diminution du contraste de contrainte associé à des dislocations dont les vecteurs de Burgers sont plus petits que ceux de la matrice (Fig. 12). L'influence de l'amplitude de b se traduit également lorsque $\Sigma$ augmente et pour des désorientations telles que $d$ reste sensiblement constant (Fig. 13).

Lorsque $\Sigma$ atteint 25 , aucun réseau de dislocations secondaires n'a pu être révélé quel que soit l'écart 


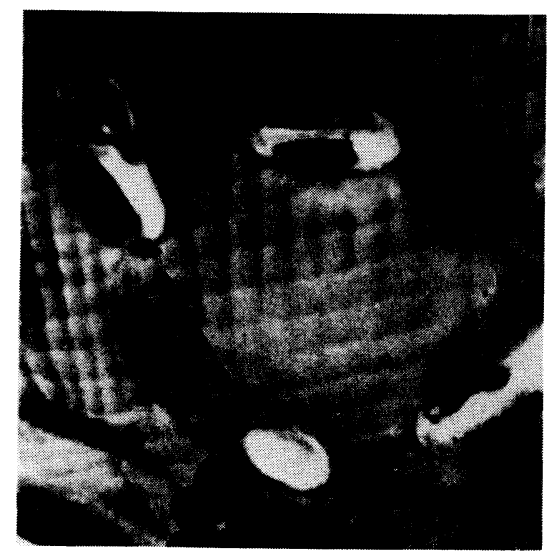

(a)

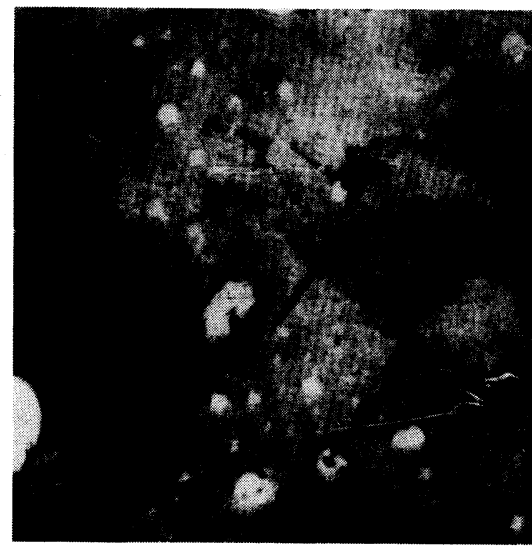

(c)

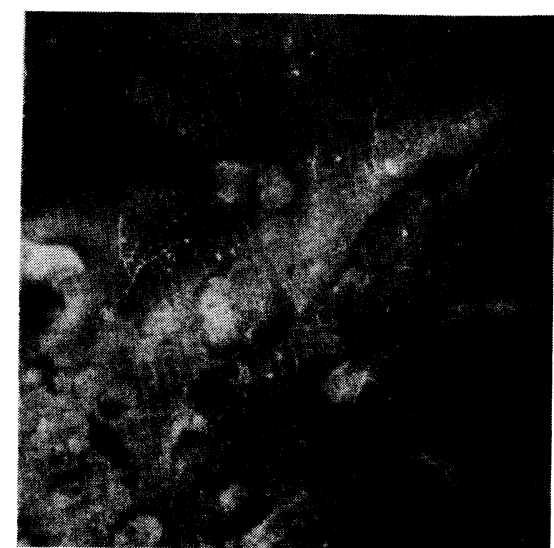

(b)

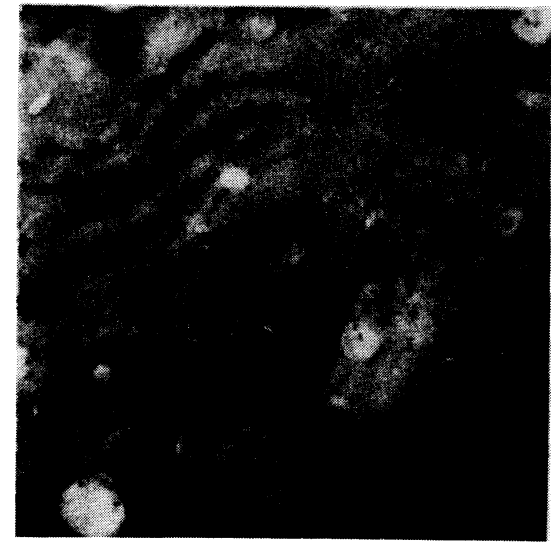

$(d)$

$\stackrel{\vdash}{0.1 \mu \mathrm{m}}$

Fig. 12. - Réseaux de dislocations secondaires dans un joint de torsion [001] de l'or $\left(\theta \# 36^{\circ} 9, \Sigma=5\right)$. Le contraste des dislocations diminue lorsque leur distance diminue c'est-à-dire lorsque $\Delta \theta$ augmente, car le vecteur de Burgers est petit [29].

[Square grids of intrinsic secondary dislocations in [001] twist boundaries in gold $\left(\Sigma=5, \theta \# 36^{\circ} 9\right)$; the visibility of GBD's decreases as their spacing decreases ( $\Delta \theta$ increases) because their Burgers vector is small [29].]

à la coïncidence $\left({ }^{5}\right)$; d'ailleurs cet écart diminue lorsque $\Sigma$ augmente (Fig. 4) en accord avec certaines prévisions énergétiques (Fig. 14) [29]. Nous avons vu, dans le chapitre précédent, que cela explique l'absence de réalité physique des dislocations secondaires dans les joints à faible désorientation $\left(\theta<16^{\circ}\right)$.

3.1.2 Joints de flexion. - a) Dislocations primaires. - $\mathrm{Si}$ on considère un joint de flexion de faible désorientation autour du même axe [001], le plan du joint étant (100), les prévisions théoriques ne sont plus aussi simples que dans le cas d'un joint de torsion. Deux modèles sont proposés (Fig. 15) [73] :

- Le joint est constitué d'une seule rangée de dislocations coin parallèles à $\langle 001\rangle$ avec $\mathbf{b}=a[100]$;

(5) Bollmann, Michaut, Sainfort ont montré l'existence, dans un acier inoxydable, de dislocations de joint de grains dont le vecteur de Burgers $|\mathbf{b}|=0,47 \AA(\Sigma=29)$ mais il s'agit de dislocations extrinsèques non périodiques [42] (cf. §3.3).
- Le joint est constitué d'un arrangement de paires de dislocations très proches l'une de l'autre, alignées également selon $\langle 001\rangle$ avec des vecteurs de Burgers respectivement égaux à $\mathbf{b}=a / 2$ [110] et $\mathbf{b}=a / 2$ [110]. Rien ne permet de prévoir quel sera le modèle opérant, ni les calculs d'énergie, ni la distance entre les dislocations : dans le second cas $\mathbf{b}_{\text {total }}=a[100]$, ce qui conduit à la même distance que dans le premier cas. Enfin le fait que dans la matrice on n'observe pas de dislocation ayant un vecteur de Burgers $a$ [100] n'élimine pas leur présence possible dans le joint de grains où elles peuvent être stabilisées.

Cette ambiguïté n'est pas levée par les observations qui confirment l'existence de rangées de dislocations parallèles à l'axe de tilt et distantes de $d=a / \theta$ (Fig. 16). L'analyse du contraste ne permet pas de se prononcer quant à l'unicité d'une rangée ou sa composition en paires de dislocations extrêmement voisines [73]. Une première complication existe donc, par rapport 


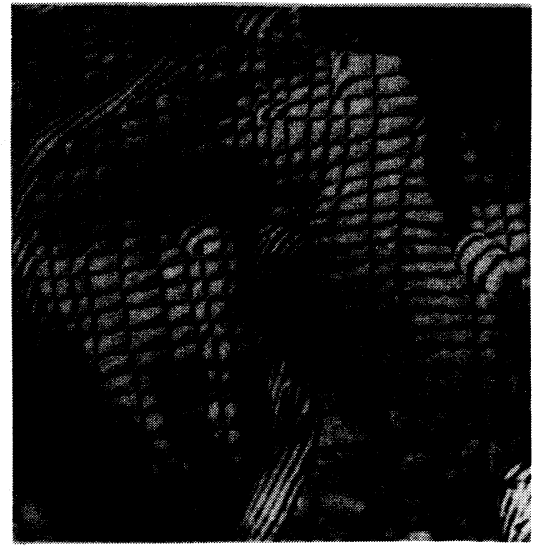

$(a)$

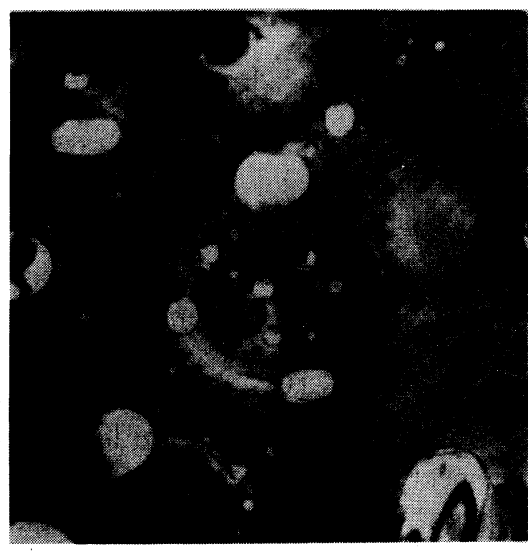

(c)

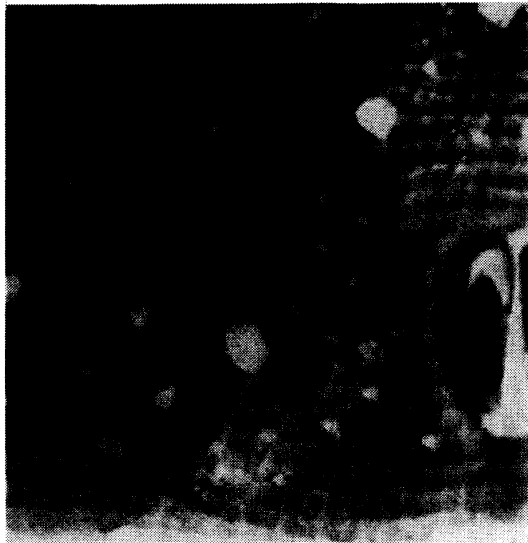

(b)

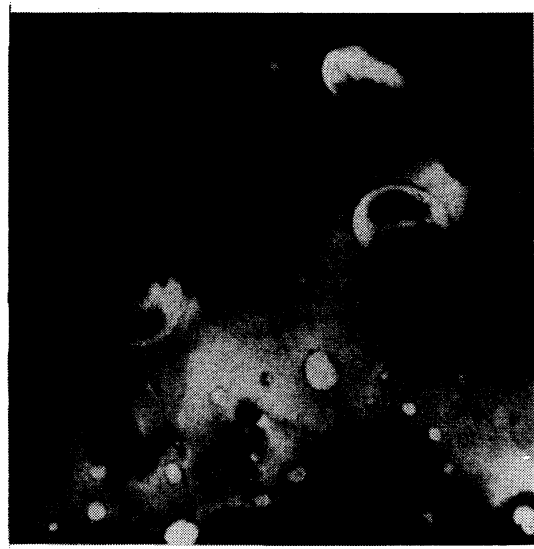

$(d)$

Fig. 13. - Réseaux de dislocations intrinsèques dans un joint de torsion [001] de l'or :

a) $\Sigma=1 \quad \theta \# 0^{\circ} \quad b=2,89 \AA$ dislocations primaires.

b) $\Sigma=5 \quad \theta \# 36^{\circ} 9 \quad b=1,29 \AA$

c) $\Sigma=13 \quad \theta \# 22^{\circ} 6 \quad b=0,80 \AA$ \& dislocations secondaires.

d) $\Sigma=17 \quad \theta \# 28^{\circ} 1 \quad b=0,70 \AA$

Noter la diminution du contraste des dislocations lorsque leur vecteur de Burgers décroît ( $\Sigma$ augmente) [29].

[Square grids of intrinsic GBD's in [001] twist boundaries in gold :
a) $\Sigma=1$
$\theta \# 0^{\circ}$
$b=2.89 \AA$ primary dislocations.
b) $\Sigma=5$
$\theta \# 36^{\circ} 9$
$b=1.29 \AA$
c) $\left.\Sigma=13 \quad \theta \# 22^{\circ} 6 \quad b=0.80 \AA\right\}$ secondary dislocations.
d) $\Sigma=17$
$\theta \# 28^{\circ} 1$
$b=0.70 \AA$

Sequence shows the decreasing visibility of the dislocations as their Burgers vector decreases ( $\Sigma$ increases) [29].]

Fig. 14. - Schéma donnant l'énergie d'un joint de torsion [001] en fonction de la désorientation $\theta$. L'écart angulaire pour lequel l'énergie reste relativement faible est d'autant plus grand que $\Sigma$ est petit [29].

[Schematic representation of energy versus twist angle for [001] twist boundaries; the angular ranges of the cusp corresponding to a relatively low energy increases as $\Sigma$ decreases [29].]

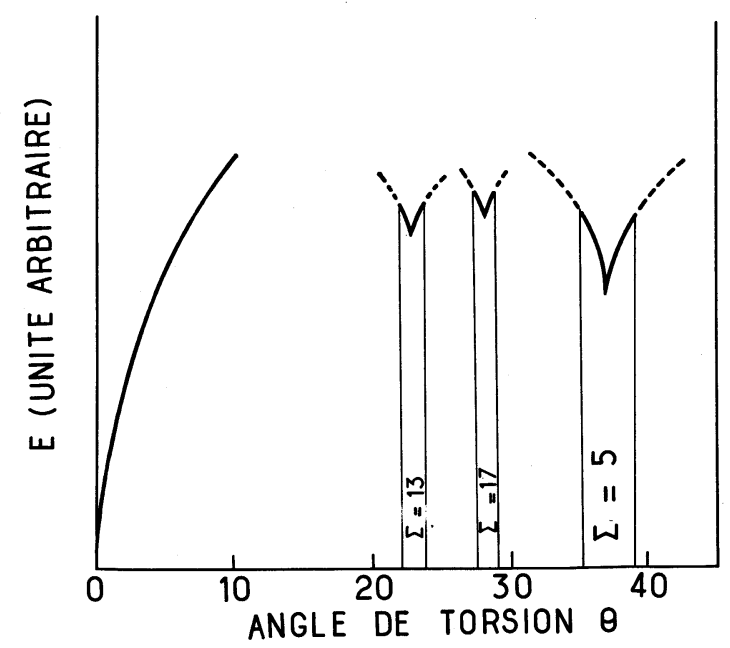




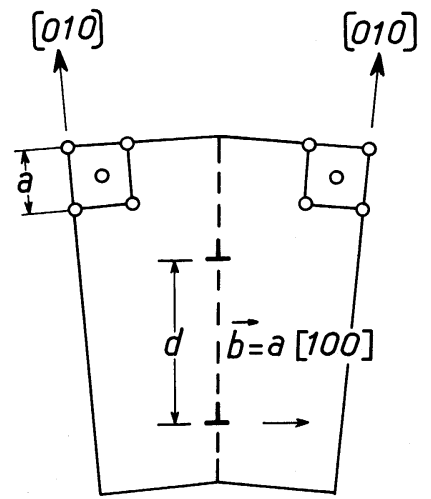

(a)

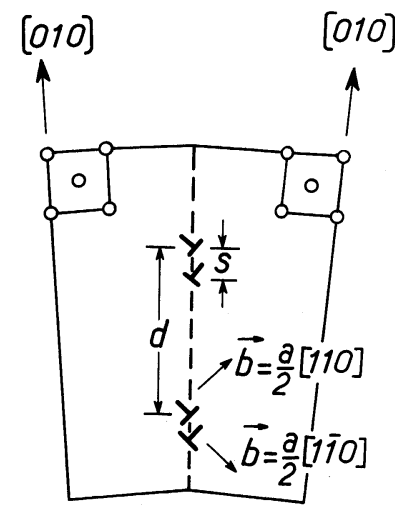

(b)
Fig. 15. - Schéma montrant les deux modèles possibles pour les dislocations primaires dans un joint de flexion [001] [73].

[Two primary dislocations models for [001] tilt boundaries [73].]

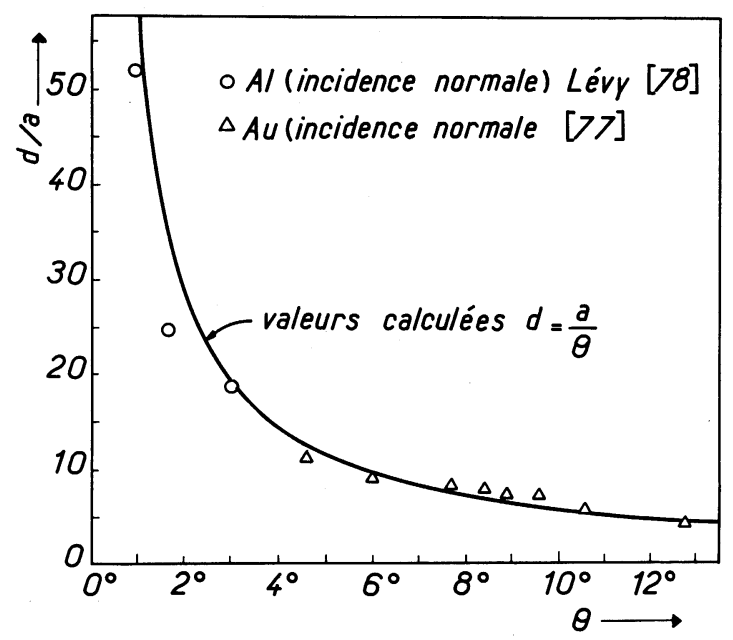

Fig. 16. - Diagramme donnant le rapport $d / a$ en fonction de la désorientation dans des joints de flexion [001] de l'or et de l'aluminium. Le bon accord entre les valeurs calculées $(-)$ et les valeurs expérimentales $\left(O\right.$ et $\triangle$ ) prouve que $\mathbf{b}_{\text {effectif }}=\mathbf{a}$ [100] [73].

[Calculated and measured $d / a$ ratio versus misorientation in [001] tilt boundaries in gold and aluminium; the good matching between the two values confirm that the effective Burgers vector $\mathbf{b}_{\mathrm{e}}=\mathbf{a}[100][73]$.]

au joint de torsion, c'est que le vecteur de Burgers du réseau de dislocations primaires peut ne pas être le vecteur de Burgers effectif mais un vecteur de Burgers possible du cristal.

Des observations plus attentives du même type de joint montrent, lorsque $\theta$ est inférieur à $9^{\circ}$ environ. que les lignes observées précédemment ont bien leur direction moyenne parallèle à l'axe de tilt mais qu'elles ont, en fait, une forme en dents de scie dont les segments sont parallèles aux directions $\langle 110\rangle$ [74]. Ces segments possèdent eux-mêmes une périodicité selon l'axe de tilt (Fig. 17). La double périodicité des rangées et des segments entraînent l'apparition de taches supplémentaires sur les clichés de diffraction.

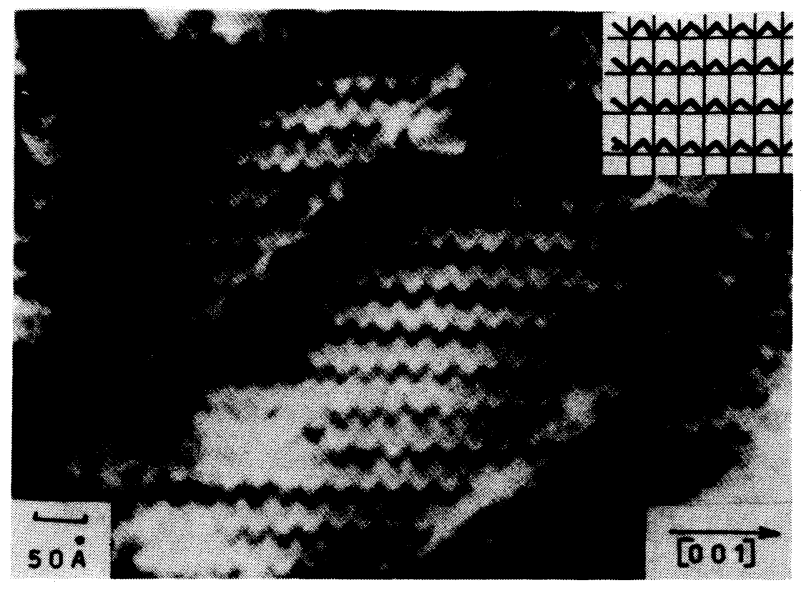

Fig. 17. - Joint de flexion [001] dans l'or $\left(\theta=6^{\circ}\right)$. Les dislocations ont leur direction moyenne parallèle à l'axe de flexion mais se décomposent en segments parallèles à $\langle 110\rangle$ [74].

$\left[[001]\right.$ tilt boundary in gold $\left(\theta=6^{\circ}\right)$; the dislocations lie on average along the tilt axis but have a serrated structure with segments parallel to $\langle 110\rangle[74]$.]

Les taches satellites dues aux rangées sont analogues à celles décrites dans le cas des joints de torsion et ont été utilisées pour imager les dislocations [74]. Quant à l'origine du second type de réflexions, aucune interprétation n'a été donnée jusqu'à présent. Lorsque $\theta$ atteint $9^{\circ}$ environ, les dislocations prennent un aspect rectiligne et, simultanément, les réflexions correspondant aux segments disparaissent. Mais le maintien des taches dues aux rangées n'exclut pas d'étendre l'existence des relaxations primaires aux joints de flexion à grand angle; des travaux sont en cours sur les joints de flexion [001] dans l'or [75]. L'interprétation de l'aspect dentelé des dislocations dans les joints de flexion à faible désorientation s'appuie sur leur tendance à se dissocier dans les plans $\{111\}$ en partielles de vecteur de Burgers $a / 6\langle 211\rangle$ et de direction parallèle à $\langle 011\rangle[74]$; ceci correspond à une diminution de l'énergie de cœur mais à une augmentation de la densité de dislocations. C'est parce que la direction de l'axe de tilt n'est pas, dans ce cas, contenue dans un plan de glissement que la décomposition suivant $\langle 110\rangle$ a lieu car elle permet alors la dissociation en dislocations partielles; chaque branche donne lieu à deux dislocations partielles de Schockley, mais deux d'entre elles situées dans des plans $\{111\}$ différents peuvent se combiner pour former une dislocation stair-rod à l'intersection des deux plans (Fig. 18) [74], par exemple :

$$
a / 6\langle 11 \overline{2}\rangle+a / 6\langle 1 \overline{1} 2\rangle \rightarrow a / 3\langle 100\rangle
$$

Lorsque $\theta$ augmente, d'une part les réactions de dissociation propres à la matrice sont moins probables [76], d'autre part, la distance entre dislocations diminue et leur interaction peut les stabiliser empêchant ou réduisant fortement leur dissociation. Notons également que les phénomènes décrits précédemment 


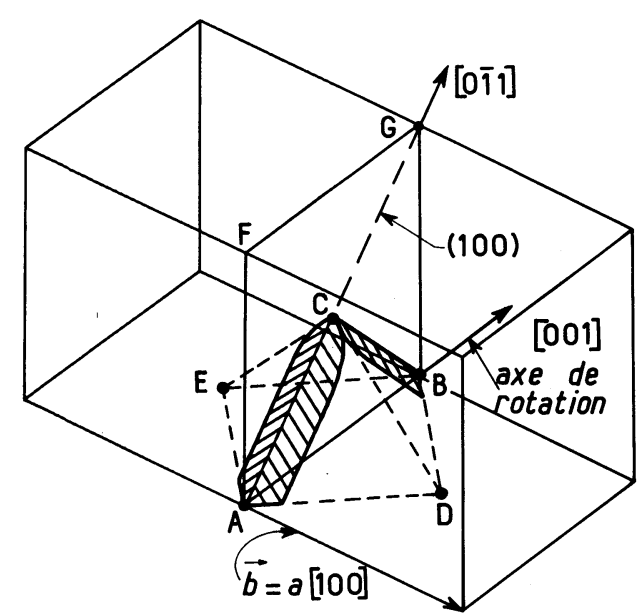

(a)

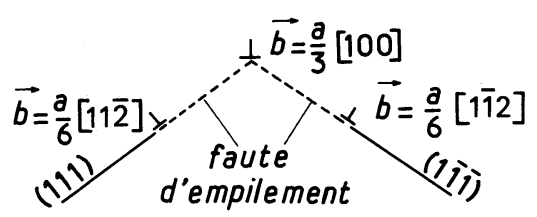

(b)

Fig. 18. - a) Modèle proposé pour expliquer le profil en dents de scie des dislocations primaires dans un joint de flexion [001]. Une dislocation selon $A B$ ne peut se dissocier, mais des segments de dislocations le long de $\mathrm{AC}$ et $\mathrm{CB}$ peuvent se dissocier dans les plans $\{111\}$ en dislocations stair rod et dislocations partielles de Shockley séparées par des fautes d'empilement; $b$ ) Vue finale de la dislocation le long de AC [74].

[a) Proposed model for serrated primary dislocation in [001] tilt boundary. A dislocation along $\mathrm{AB}$ cannot dissociate but dislocation segments along AC and CB can dissociate on $\{111\}$ planes into stair rod dislocations and Shockley partial dislocations separated by stacking faults. $b$ ) End view of dislocation looking along AC [74].]

sont d'autant plus possibles que le métal a une énergie de faute d'empilement basse, ce qui est bien le cas de l'or.

En conclusion, dans le cas des joints de flexion à faible désorientation, l'application du réseau « $O$ " ne rend pas directement compte de l'arrangement des dislocations primaires; les deux restrictions évoquées ne sont pas dues à la limitation des techniques mais à des insuffisances du modèle géométrique; ce modèle continue à servir de base mais l'interprétation de la structure du joint est beaucoup plus complexe.

b) Dislocations secondaires. - Notons tout d'abord que, dans le système cubique, les indices de coïncidence, les réseaux C.S.L. et D.S.C. sont les mêmes pour un angle de rotation donné quel que soit le caractère du joint, de torsion ou de flexion (ce caractère est déterminé par l'orientation du plan du joint dans le réseau de coïncidence). Les vecteurs de Burgers possibles des dislocations secondaires sont donc donnés dans le tableau I (cf. § 3.1.1). Une condition supplémentaire intervient, dans le cas des joints de flexion : les vecteurs de Burgers sont les vecteurs du réseau D.S.C. les plus petits possibles possédant les composantes normales au plan du joint les plus grandes possibles.

Remarquons également que le vecteur $\mathbf{b}_{3}$ parallèle à l'axe de tilt et contenu dans le plan du joint ne peut être opérant dans un pur joint de flexion.

Précisons pour $\Sigma=5$ les considérations théoriques qui en résultent pour deux joints de flexion $\langle 001\rangle$ ayant respectivement comme plan du joint (Fig. 19) [77] :

- (310) de la matrice ou (100) du réseau de coïncidence $\left(\theta=36^{\circ} 9\right)$,

- (210) de la matrice ou (110) du réseau de coïncidence $\left(\theta=53^{\circ} 1\right)$.

Lorsque $\theta=36^{\circ} 9$, un seul vecteur de Burgers répond au critère précédent, l'écart à la coïncidence doit donc être compensé par un réseau de dislocations secondaires parallèles à l'axe de tilt et de vecteur de Burgers $\mathbf{b}_{1}=a / 10\langle 310\rangle$.

Lorsque $\theta=53^{\circ} 1$, la situation est plus complexe puisque $\mathbf{b}_{1}$ et $\mathbf{b}_{2}$ sont à $45^{\circ}$ du plan du joint et que la somme de leurs composantes normales au joint correspond au vecteur $\mathbf{b}_{\mathrm{e}}$ du réseau D.S.C. Un cas analogue a déjà été rencontré pour les joints à faible désorientation (cf. § 3.1.2.a). Il est alors impossible de distinguer a priori entre deux possibilités :

- l'existence d'un réseau unique de dislocations coin parallèles à $\langle 001\rangle$ et de vecteur de Burgers

$$
\mathbf{b}_{\mathrm{e}}=a / 5\langle 210\rangle=\mathbf{b}_{1}+\mathbf{b}_{2} ;
$$

- l'existence de deux réseaux extrêmement rapprochés de dislocations parallèles à $\langle 001\rangle$ possédant respectivement les vecteurs de Burgers $\mathbf{b}_{1}$ et $\mathbf{b}_{2}$.

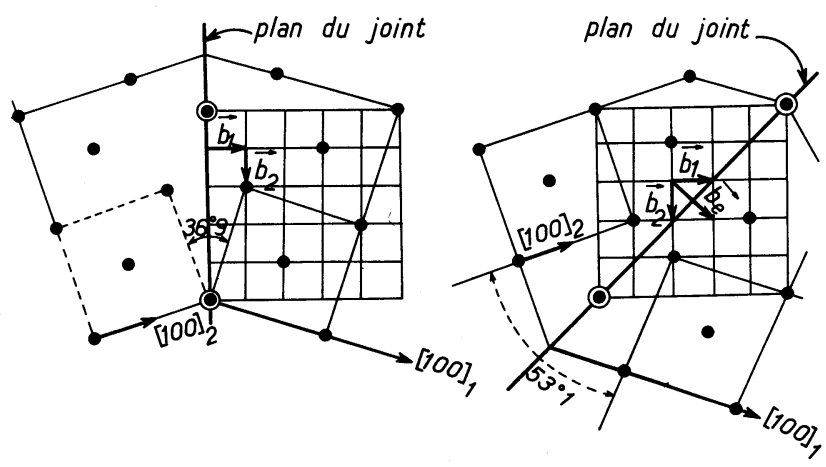

Fig. 19. - Schémas montrant les vecteurs de Burgers possibles des dislocations secondaires dans un joint de flexion [001] $\Sigma=5$; le vecteur de Burgers effectif doit être perpendiculaire au plan du joint : $a$ ) Le plan du joint est un plan $\{310\}$ des cristaux $\theta=36^{\circ} 9$; $\left.\mathbf{b}_{\mathbf{e}}=\mathbf{b}_{1}=\mathbf{a} / 10\langle 310\rangle ; b\right)$ Le plan du joint est un plan $\{210\}$ des cristaux, $\theta=53^{\circ} 1 ; \mathbf{b}_{\mathrm{e}}=\mathbf{b}_{1}+\mathbf{b}_{2}=\mathbf{a} / 5\langle 210\rangle$.

[Diagram showing the possible Burgers vectors of secondary dislocations in [001] tilt boundary $(\Sigma=5)$; the effective Burgers vector must be perpendicular to the boundary plane $: a$ ) boundary plane is $\{310\}$ in crystals, $\theta=36^{\circ} 9 ; \mathbf{b}_{\mathbf{e}}=\mathbf{b}_{1}=a / 10\langle 310\rangle$. b) Boundary plane is $\{210\}$ in crystals, $\theta=53^{\circ} 1$;

$$
\mathbf{b}_{\mathbf{e}}=\mathbf{b}_{1}+\mathbf{b}_{2}=\mathbf{a} / 5\langle 210\rangle \text {.] }
$$


L'expérience ne permet pas de lever cette ambiguité théorique d'autant plus que les rangées de dislocations secondaires observées dans tous les joints de flexion à grand angle présentent des irrégularités d'orientation et d'espacement (Fig. 20) [77] dues vraisemblablement à l'impossibilité de contrôler rigoureusement la déviation $\Delta \theta$ à la coïncidence. La préparation des échantillons entraîne, en effet, des variations locales de l'angle de flexion qu'il est impossible de mesurer en microscopie électronique puisque, dans ce cas, l'axe de rotation est parallèle au plan de la lame. Les incertitudes sur $d$ et sur $\Delta \theta$ ne permettent pas de vérifier la valeur absolue du vecteur de Burgers et, par conséquent, de relier la présence des rangées sans ambiguité à un écart de flexion par rapport à la coïncidence exacte. Seule, la présence sur certaines lignes de dislocations d'un contraste symétrique permet de leur attribuer un caractère coin (cf. $\S 4.1 .1$ ); de plus, dans ce cas, les déviations de torsion introduites inévitablement lors de la fabrication des échantillons donnent lieu à des dislocations extrinsèques au contraste net (Fig. 20).

Il faut souligner cependant que, dans certains joints de flexion et pour des conditions d'observation où les dislocations coin sont éteintes (g// u) (cf. §4.1.1) un réseau de dislocations vis a pu être observé, l'écart à la coïncidence ayant, en général, un caractère mixte [78].

La mise en évidence des relaxations secondaires attribuables à un écart de flexion par rapport à la coïncidence est compliquée pour deux raisons : non seulement b est petit (comme dans le cas des dislocations secondaires vis) mais, de plus, les conditions de contraste sont difficilement réalisables. Il est alors intéressant de préciser les résultats obtenus sur les joints de grains dont le plan est parallèle à la lame

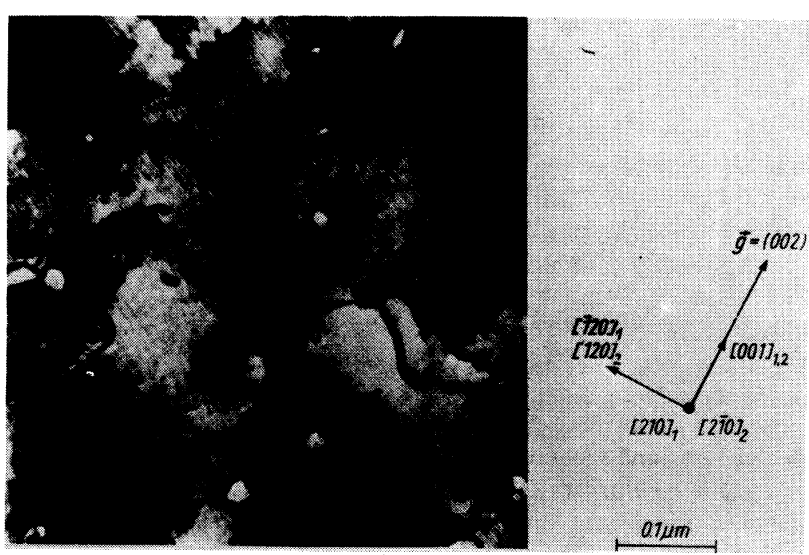

Fig. 20. - Rangées irrégulières de dislocations secondaires dans un joint de flexion [001] de désorientation voisine de $53^{\circ} 1$. Les lignes sombres sont des dislocations extrinsèques associées à une faible composante de torsion [77].

[Irregular arrays of secondary dislocations in [001] tilt boundary $\left(\theta \# 22^{\circ} 6\right)$. Dark coarse lines are extrinsic dislocations associated with small twist component [77].] mince par ceux déduits de l'observation de joints perpendiculaires à la lame [77, 67].

Notons enfin que l'aspect dentelé des dislocations coin primaires ne se retrouve pas sur les dislocations secondaires en accord avec l'interprétation donnée précédemment pour des angles supérieurs à $10^{\circ}$ [76].

3.1.3 Conclusion. - Tous les résultats concernant les joints spéciaux considérés comme de purs joints de torsion ou de flexion autour d'axes de faibles indices montrent que :

- le formalisme de Bollmann peut toujours constituer une base pour l'interprétation de la structure intrinsèque de ces joints,

- mais, bien que ces joints représentent des cas relativement simples, il existe des limites à la réalité physique, et même géométrique, de ce formalisme.

Or, en fait, la déviation à la coïncidence présente le plus souvent un caractère mixte, même dans les bicristaux préparés en vue d'obtenir une désorientation unique, difficilement réalisable. On est en droit de se demander quelle sera l'utilisation possible d'un modèle présentant déjà des restrictions pour les joints les plus spéciaux. Cependant, la périodicité signalée précédemment dans une interface mixte [78] suggère le maintien d'un certain ordre. On est en présence d'un second type de joints spéciaux décrits soit par le modèle dit du Plane Matching [79] soit, plus récemment, par l'existence d'une direction axiale de coïncidence C.A.D. [80].

3.2 Joints spéciaux "Plane Matching " ou "C.A.D. ". - Initialement proposé par Pumphrey [79], le modèle du Plane Matching permet de rendre compte de l'existence de joints possédant une seule périodicité sans référence au réseau de coïncidence. C'est cette présentation que nous adopterons en premier lieu, puis nous montrerons comme de nombreux auteurs se sont efforcés de le prouver depuis $[71,72,81,82,83]$, que le modèle du P.M. (Plane Matching) s'explique à partir du formalisme de Bollmann; la terminologie C.A.D. semble alors mieux appropriée.

3.2.1 Modèle du Plane Matching. - - De part et d'autre d'un joint, il existe au moins deux ensembles de plans de mêmes indices, de densité atomique relativement élevée, qui sont en "bon accord». Les traces de ces plans dans le joint correspondent à des rangées très légèrement inclinées les unes par rapport aux autres (Fig. 21a) et dont les espacements diffèrent quelque peu (Fig. 21b). Ces traces étant des régions de plus forte densité atomique, autour d'elles des relaxations interviennent et leurs champs de contrainte peuvent être visualisés en microscopie électronique par un effet de Moiré; les lignes d'opacité correspondent aux régions qui, périodiquement, présentent le plus fort désordre atomique dans le plan du joint. Il convient de bien distinguer ce faux Moiré traduisant la structure propre de l'interface de l'effet de Moiré habituel dû la superposition de deux cristaux. 

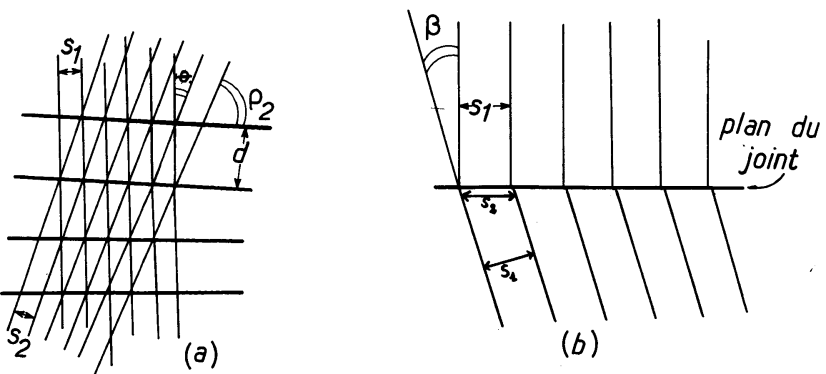

(b)

Fig. 21. - Schémas permettant d'expliquer le modèle du Plane Matching [79] : a) - : traces légèrement désorientées d'un angle $\theta$ dans le plan du joint des plans de mêmes indices des cristaux 1 et 2 adjacents; - : Franges du type Moiré observées distantes de $d . b$ ) Les espacements, dans le plan du joint, des traces des plans $\{h k l\}$ des deux cristaux diffèrent.

[Schematic explanation of the plane matching model [79] :a) Slightly mismatched traces in the boundary of planes impinging from adjoining crystals 1 and $2(-) ; d$ : spacing of the Moire fringes $(-) ; b)$ In the boundary plane, the spacings of these traces are different.]

La distance des franges observées "répond à la formule générale [84] :

$$
d=\frac{s_{1} s_{2}}{\left(s_{1}^{2}+s_{2}^{2}-2 s_{1} s_{2} \cos \theta\right)^{1 / 2}} .
$$

La rotation $\rho$ entre les franges de Moiré observées et les traces des plans du grain 1 sur l'interface est donnée par:

$$
\sin \rho=\frac{s_{1} \sin \theta}{\left(s_{1}^{2}+s_{2}^{2}-2 s_{1} s_{2} \cos \theta\right)^{1 / 2}}
$$

ou $\sin \rho=d \sin \theta / s_{2}$.

Bien qu'en théorie tout ensemble de plans peut donner lieu à un tel phénomène, le modèle n'a été directement appliqué, dans le système C.F.C., que pour le bon registre des plans $\{200\},\{220\},\{111\}$ $[78,85]$ et dans le système C.C. pour les plans $\{110\}$ [86].

On peut ainsi montrer simplement que la structure d'un joint de torsion faiblement désorienté autour de $\langle 001\rangle$ s'interprète en considérant l'accord approché des plans $\{111\}$; ces plans interceptent le joint selon des traces $\langle 110\rangle$ distantes de $a / 2\langle 110\rangle$ et donnent donc lieu à des franges de faux Moiré dont l'espacement est

$$
d=\frac{a \sqrt{2}}{4 \sin \theta / 2} .
$$

On retrouve bien entendu la distance des dislocations primaires (cf. § 2.2.1) :

avec

$$
\begin{aligned}
d & =\frac{b}{2 \sin \theta / 2} \\
b & =a \sqrt{2} / 2 .
\end{aligned}
$$

De la même façon, la distance des lignes périodiques observées par Levy [78] dans un joint de flexion $\langle 001\rangle$ possédant un écart mixte à la coïncidence $(\Sigma=5)$ est contrôlée par la composante de torsion $\alpha$; elle correspond à la distance de franges de Moiré obtenues à partir du relativement bon accord des traces des plans $\{200\}$ :

$$
d=\frac{a}{4 \sin \alpha / 2} .
$$

Dans ce cas, le résultat diffère de celui obtenu en considérant les dislocations secondaires de vecteurs $\mathbf{b}_{1}$ et $\mathbf{b}_{2}(a / 10\langle 310\rangle)$ observées par Schober et Balluffi [77] mais satisfait à la relation :

$$
d=\frac{b}{2 \sin \Delta \theta / 2} \text { si } \quad b=a\langle 001>/ 2 \quad \text { et } \quad \Delta \theta=\alpha .
$$

Remarquons que ce vecteur $a\langle 001\rangle / 2$ est extrêmement voisin du vecteur $\mathbf{b}_{3}$ du réseau D.S.C. lorsque $\Sigma=5$ et devient rigoureusement $b_{3}$ lorsque $\Sigma$ augmente pour un angle de flexion autour de [001] différent de $36^{\circ} 9$.

Signalons enfin que, récemment, Schindler, Clemens et Balluffi ont déterminé l'existence de phénomènes périodiques dans des joints de flexion [001] de l'or pour $\theta \# 42^{\circ}$ (désorientation éloignée de toute coïncidence simple) pour des écarts supplémentaires de torsion allant jusqu'à $\dot{4}^{\circ}$ (Fig. 22) [85] et pour des

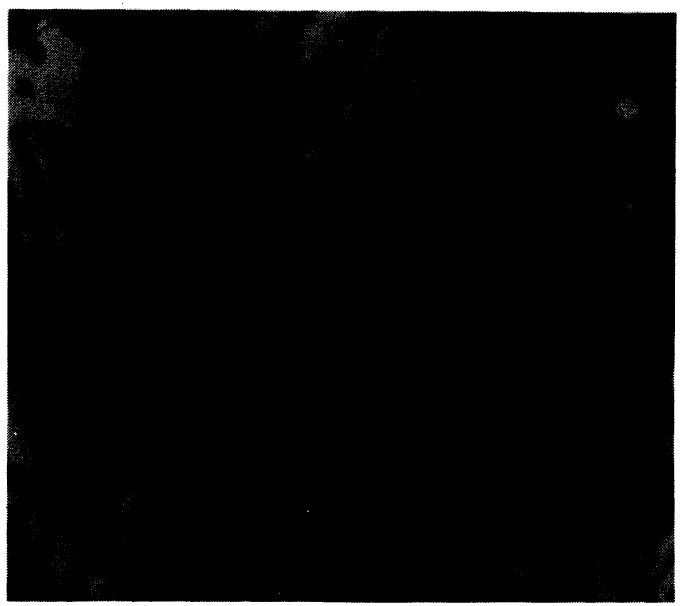

Fig. 22. - Mise en évidence d'un phénomène périodique dans un joint de flexion asymétrique [001] $\left(\theta=42^{\circ}\right)$ avec un plan du joint $\{002\}$ dans un cristal et possédant une légère composante de torsion $\alpha$ autour d'un axe perpendiculaire au plan du joint (voir Fig. 23) [85].

[Periodic phenomena in an asymmetric [001] tilt boundary $\left(\dot{\theta}=42^{\circ}\right)$ with the boundary plane oriented parallel to $\{002\}$ in one lattice and possessing a small twist component $\alpha$ around an axis perpendicular to the boundary plane (see Fig. 23) [85].]

écarts de flexion autour d'un axe perpendiculaire à l'axe de tilt fondamental allant jusqu'à $14^{\circ}$ [87]; ils ont pu interpréter cette périodicité comme résultant également du bon accord des traces des plans $\{200\}$ (Fig. 23). 


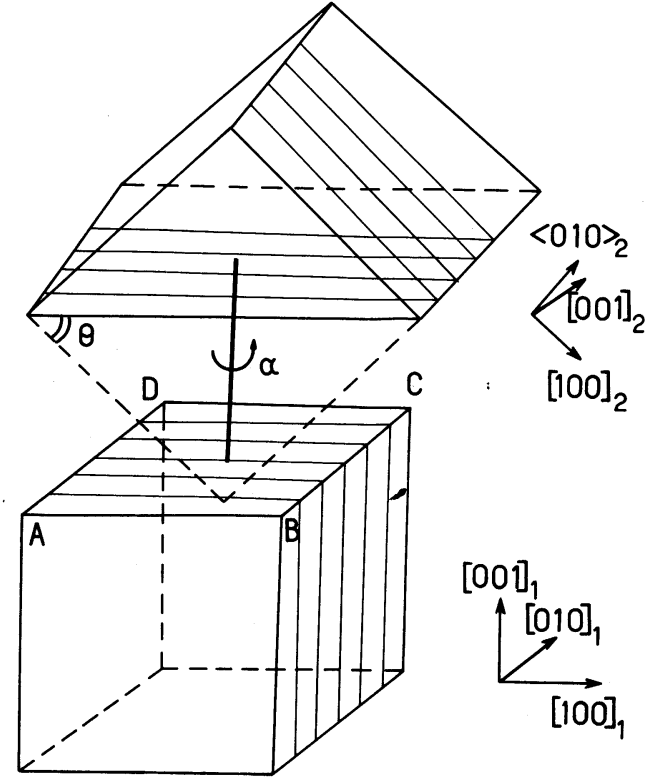

Fig. 23. - Le phénomène périodique observé (Fig. 22) dans un joint de flexion [001] $\left(\theta=42^{\circ}\right)$ possédant une composante supplémentaire de torsion peut s'expliquer par le bon accord relatif des traces des plans $\{200\}$ des cristaux adjacents [85].

[The previous periodic phenomenon can be explained by the relatively good matching, in the boundary, of the $\{200\}$ planes of the two crystals [85].]

On est frappé par le fait que l'approche du Plane Matching ne rend compte des franges observées qu'en utilisant un système de plans bien particulier, or, pour une même interface, d'autres plans peuvent théoriquement s'intercepter avec un bon accord [81]. C'est-à-dire que le modèle permet d'expliquer l'observation mais pas de prévoir la structure du joint puisqu'aucun critère ne permet de décider, a priori, quels plans considérer.

Nous allons voir que ce modèle peut être compris comme une extension de la théorie du réseau de coïncidence et que les prévisions sont alors possibles.

\subsubsection{Modèle du C.A.D. (Coincidence Axis Direc-} tion). - Ce modèle étend l'analyse des relaxations secondaires, corrélée à la définition d'un réseau D.S.C. aux joints mixtes, voisins d'une position de coïncidence, même si $\Sigma$ est élevé. Quel que soit le type d'écart, torsion ou flexion, qui s'ajoute à la désorientation fondamentale autour d'un axe $R$, les vecteurs de base du réseau D.S.C. seront tels que :

- $\mathbf{b}_{1}$ et $\mathbf{b}_{2}$ sont contenus dans un plan perpendiculaire à $R$ et leur longueur varie sensiblement comme $\Sigma^{-1 / 2}[71,80]$;

- $\mathbf{b}_{3}$ est sensiblement parallèle à l'axe de rotation $R$, sa longueur est pratiquement indépendante de $\Sigma$ et voisine d'une distance interéticulaire. Si on considère, par exemple, un joint de flexion fortement désorienté autour d'un axe $R$ et possédant une légère composante de torsion autour d'un axe perpendiculaire au plan du joint, aucune dislocation coin ne peut être révélée puisque $\mathbf{b}_{1}$ et $\mathbf{b}_{2}$ sont infiniment petits ( $\Sigma$ élevé), par contre l'apparition d'un réseau de dislocations vis parallèles à l'axe de rotation fondamental $R$ (et non pas à l'axe de torsion) et de vecteur de Burgers $\mathbf{b}_{3}$ peut toujours s'expliquer sur la base du réseau D.S.C. Les résultats de Levy et de Schindler cités précédemment trouvent ici leur interprétation; compte tenu des valeurs de $\mathbf{b}_{3}$, il n'est pas étonnant que les écarts supplémentaires puissent être supérieurs à ceux correspondant à l'axe de flexion fondamental. Le modèle du Plane Matching revient donc à une coïncidence selon un axe d'où son appellation nouvelle C.A.D. alors que le modèle du C.S.L. correspond à une périodicité bidimensionnelle dans le plan du joint. Notons que dans le cas du C.S.L., il y a coïncidence de rangées atomiques dans l'interface alors que dans le cas du C.A.D., il y a coïncidence des traces des plans qui ne sont pas nécessairement des rangées d'atomes de faibles indices ; il sera donc, $a$ priori, plus probable de trouver une telle périodicité dans les joints au hasard.

On peut dire que le modèle du Plane Matching équivaut au modèle du C.S.L. lorsque $\Sigma \rightarrow \infty$. Une preuve de l'identité des deux modèles a été donnée récemment par Gronsky en utilisant la technique du lattice imaging des plans en bon accord au voisinage d'une interface entre deux phases [88] : à l'aplomb de chaque dislocation localisée dans le joint et visualisée par l'existence d'un plan supplémentaire se superpose une frange de Moiré (Fig. 24).

Malgré cette identité reconnue, une polémique existe sur la généralisation des deux approches : le modèle du Plane Matching est-il plus général que le modèle du C.S.L. ou réciproquement ? La réponse à cette question diffère selon qu'on considère la réalité physique ou la réalité géométrique du modèle. D'un point de vue physique, le modèle des traces de plans

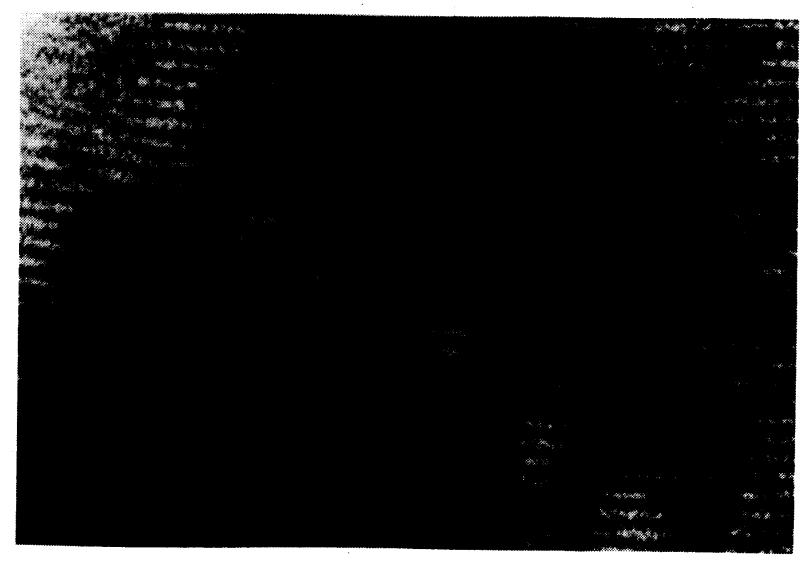

Fig. 24. - Image en microscopie électronique à haute résolution des plans en bon accord relatif dans un joint entre deux phases; chaque flèche indique l'interruption d'une frange des réseaux associée à une dislocation et localisée dans une bande de Moiré optiquement dense [88].

[High resolution image of planes slightly mismatched in an interphase boundary; arrows indicate terminating fringes associated with a dislocation localized in high density Moiré bands [88].] 
en bon accord peut apparaître plus général : on vient de voir qu'il explique des phénomènes périodiques dans des joints interphases [88], rien n'exclut donc la possibilité de l'étendre à l'accord de plans d'indices différents orientés de telle façon par rapport au plan du joint que $s_{1}$ et $s_{2}$ diffèrent peu [89]. Mais cette généralisation est a posteriori. Géométriquement, il est toujours possible de trouver un réseau de coïncidence à $\Sigma$ élevé donc un vecteur $\mathbf{b}_{3}$. Seule, la dimension de ce vecteur (dépendant des indices de l'axe de rotation fondamental) peut limiter l'observation $\mathrm{du}$ réseau de dislocations secondaires qui lui est attaché. A priori, tout phénomène périodique peut donc être interprété sur la base du formalisme de Bollmann.

3.2.3 Conclusions. - La possibilité de révéler des dislocations secondaires dans un joint mixte plus ou moins proche de la coïncidence dépendra de son caractère fondamental. On peut ainsi observer :

- Dans un joint essentiellement de torsion (Fig. 25) :

- une grille (ou un des réseaux de la grille) de dislocations vis de vecteurs de Burgers $\mathbf{b}_{1}$ et $\mathbf{b}_{2}$ et d'orientations parallèles à ces vecteurs,

- un arrangement périodique de dislocations coin parallèles à un axe $F$ contenu dans le plan du joint et de vecteurs de Burgers $\mathbf{b}_{3}$ perpendiculaire à ce plan.

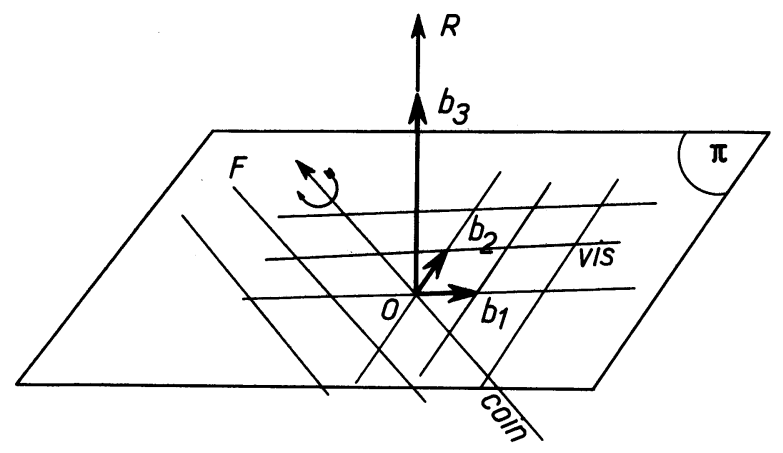

Fig. 25. - Schéma montrant les dislocations secondaires qu'il est possible d'observer dans un joint mixte essentiellement de torsion : - dislocations vis de vecteurs de Burgers $\mathbf{b}_{1}$ et $\mathbf{b}_{2}$

- dislocations coin de vecteur $\mathbf{b}_{3}$ et parallèles à l'axe de flexion supplémentaire $F$.

[Schematic representation of the possible secondary dislocations in a high angle twist boundary possessing a small twist deviation : - screw dislocations with Burgers vectors $\mathbf{b}_{1}$ and $\mathbf{b}_{2}$

- edge dislocations with Burgers vector $\mathbf{b}_{3}$ running parallel to the tilt axis $F$.]

- Dans un joint essentiellement de flexion (Fig. 26) : - un réseau de dislocations coin parallèles à l'axe de tilt $R$ et dont le vecteur de Burgers est l'un des vecteurs $\mathbf{b}_{1}$ ou $\mathbf{b}_{2}$ (ou leur somme), vecteurs contenus dans un plan perpendiculaire à $R$,

- un réseau de dislocations vis également parallèles à l'axe de tilt $R$, de vecteur de Burgers $\mathbf{b}_{3}$ provoqué par une faible composante de torsion

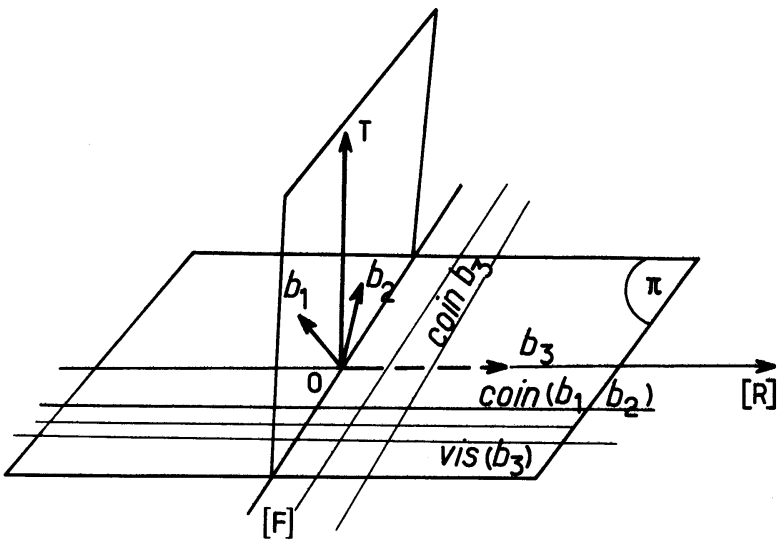

Fig. 26. - Schéma montrant les dislocations secondaires qu'il est possible d'observer dans un joint mixte à caractère fondamental de flexion :

- dislocations coin de vecteur $\mathbf{b}_{1}$ ou $\mathbf{b}_{2}$ (ou leur somme) et parallèles à l'axe de flexion fondamental $R$,

- dislocations vis de vecteur $\mathbf{b}_{3}$ paralleles à l'axe de torsion $R$,

- dislocations coin de vecteur $\mathbf{b}_{3}$ parallèle à un axe de flexion secondaire $F \perp R$

[Schematic representation of the possible secondary dislocations in a high angle tilt boundary possessing small twist and tilt (around a secondary axis) deviations :

- edge dislocations with Burgers vector $\mathbf{b}_{1}$ or $\mathbf{b}_{2}$ (or $\mathbf{b}_{1}+\mathbf{b}_{2}$ ) and running parallel to the fundamental tilt axis $R$,

- screw dislocations with Burgers vector $b_{3}$ along a twist axis $R$, - edge dislocations with Burgers vector $\mathbf{b}_{3}$ parallel to a secondary tilt axis $F \perp R$.]

autour de la normale au plan du joint OT, - un réseau de dislocations coin parallèles à un axe de tilt $\mathrm{OF}$, lui-même perpendiculaire à $R$, de vecteur de Burgers $b_{3}$ provoqué par une composante supplémentaire de flexion autour de OF.

Un grand nombre de joints peuvent, en principe, se décrire comme le produit de deux rotations autour d'axes perpendiculaires dont l'une est nettement plus importante que l'autre, mais la détection des dislocations associées à chaque composante dépendra de la dimension des vecteurs de base du réseau D.S.C. et de l'orientation du plan du joint. En particulier, si l'axe fondamental ne peut pas être un axe simple, $\mathbf{b}_{3}$ est trop petit pour espérer trouver au moins une périodicité : le joint est alors du type général.

\section{3 LA STRUCTURE HORS D'ÉQUILIBRE DU JOINT. -} La structure extrinsèque ou hors d'équilibre du joint résulte de l'interaction des dislocations de la matrice avec la structure intrinsèque ou d'équilibre du joint de grain. Elle consiste en la présence dans l'interface de dislocations extrinsèques (E.G.B.D.'s) isolées dont les champs de contrainte ne s'annulent pas à grande distance; ces dislocations ne modifient pas globalement la désorientation entre les grains adjacents. $\mathrm{Si}$ l'interaction de nombreuses dislocations de matrice avec le joint se traduit par un empilement régulier de dislocations entraînant un changement de déso- 
rientation, alors les dislocations formées dans le joint de grains entrent dans sa structure intrinsèque en la modifiant (cf. § 2.2.2) [90].

On conçoit aisément que les réactions des dislocations du réseau avec le joint et que la stabilité des E.G.B.D's qui en résulte diffèrent selon la température et le moment auxquels s'effectuent les interactions : dislocations extrinsèques formées lors de la fabrication de l'échantillon (soudage des lames minces - recristallisation), introduites par déformation à température ambiante dans un joint immobile ou lors de la migration des interfaces à température relativement élevée $[38,51,52]$.

L'observation des dislocations extrinsèques est fonction du type de joint étudié : joint spécial ou joint général. L'étude de bicristaux orientés sur lesquels la structure intrinsèque est visible permet mieux l'analyse des réactions de dissociation des dislocations des grains mais les contrastes sont quelque peu masqués par le fond continu des réseaux périodiques, ce qui limite la détection de dislocations à faible vecteur de Burgers. Dans les joints généraux, ne présentant pas de structure interne mais qui peuvent correspondre à des indices de coïncidence pas trop élevés, les dislocations isolées ont un contraste bien marqué dont l'analyse, difficile, peut cependant permettre d'atteindre le vecteur de Burgers (cf. $\$ 4.1$ ).

Quelque soit le type d'interface, le problème se pose en termes de dissociation ou non des dislocations de matrice en dislocations "parfaites" du joint de grains, ce qui peut se traduire par deux questions :

- Dans les joints de faible désorientation, y a t-il conservation ou non du vecteur de Burgers du grain?

- Dans les joints à forte désorientation, les vecteurs de Burgers des dislocations extrinsèques sont-ils des vecteurs de base du réseau D.S.C. ?

En passant en revue quelques résultats concernant différents types de joints, nous allons tenter de dégager une réponse à ce problème.

3.3.1 Joints spéciaux de torsion [001]. - Lorsque la désorientation est extrêmement faible $\left(\theta<1^{\circ}\right)$, peu de dislocations extrinsèques se superposent au réseau d'équilibre dont l'irrégularité, due à l'erreur relative sur $\theta$ et aux faibles forces d'attraction entre rangées intrinsèques, semble seulement aggravée après déformation [91]. Il est vraisemblable que les dislocations de matrice traversent ce joint sans difficulté puisque les plans de glissement coïncident sensiblement de part et d'autre.

Lorsque $\theta$ est voisin de $2^{\circ}$, quelques rares dislocations extrinsèques apparaissent alignées selon [110] et présentent une forme en dents de scie (Fig. 27a) [91]. Cette configuration a été analysée par Schober et Balluffi [39] en utilisant la procédure de Frank [40].

Considérons, par exemple une dislocation de matrice de vecteur de Burgers $\mathbf{b}=a / 2$ [101] qui intercepte un réseau de dislocations vis de vecteurs $\mathbf{b}_{1}=a / 2[\overline{110} 0]$ et $\mathbf{b}_{2}=a / 2[\overline{1} 10]$. L'existence des seg-

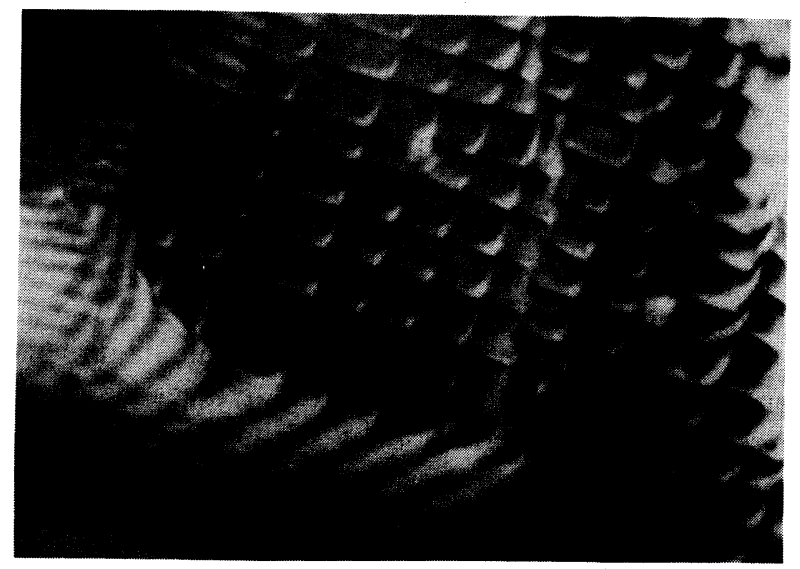

2

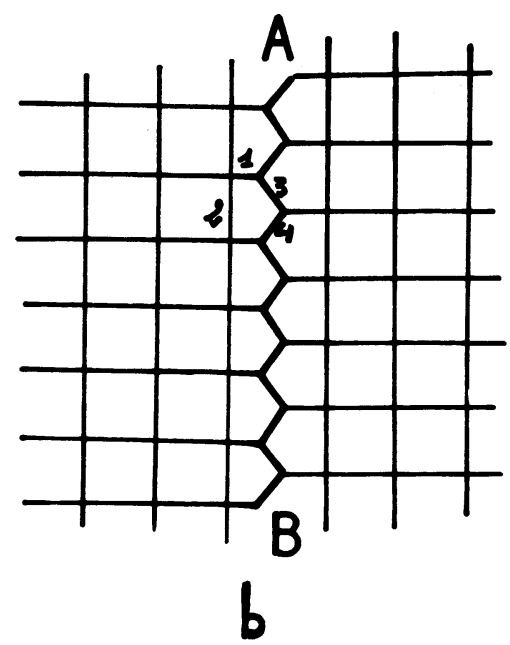

Fig. 27. - $-a$ ) Dislocation extrinsèque $\mathrm{AB}$ orientée selon une direction moyenne $\langle 110\rangle$ dans un joint de torsion [001] faiblement désorienté. Aspect en dents de scie. b) Schéma de la configuration de la dislocation le long de AB (cf. texte).

[a) Extrinsic dislocation lying along $\langle 110\rangle$ in a [001] low angle twist boundary with a serrated configuration. $b$ ) Schematic diagram of extrinsic dislocation configuration observed along $\mathrm{AB}$ (see $\S 3.3 .1)$.]

ments 3 et 4 le long de la dislocation $A B$ extrinsèque s'interprète en considérant les réactions suivantes (Fig. 27b) :

$$
\begin{aligned}
& \mathbf{b}+\mathbf{b}_{2}=\mathbf{b}_{3}=a / 2[01 \overline{1}] \\
& \mathbf{b}_{1}+\mathbf{b}_{3}=\mathbf{b}_{4}=a / 2[\overline{1} 0 \overline{1}] .
\end{aligned}
$$

Les vecteurs $\mathbf{b}_{3}$ et $\mathbf{b}_{4}$ sont à $45^{\circ} \mathrm{du}$ plan du joint. Le déplacement de la grille de dislocations intrinsèques le long de $\mathrm{AB}$ d'une distance égale à $L / 2(L$ : distance entre dislocations) s'explique par l'intervention d'un vecteur de Burgers effectif $\mathbf{b}_{\mathrm{e}}$ avec :

$$
\mathbf{b}_{\mathrm{e}}=\mathbf{b}+1 / 2\left(\mathbf{b}_{1}+\mathbf{b}_{2}\right)=a[00 \overline{1}] .
$$

Ce vecteur perpendiculaire au plan du joint rend compte de la stabilité des dislocations extrinsèques. 
- Quand $\theta$ augmente, le réseau de dislocations primaires devient trop serré pour distinguer la configuration des dislocations hors d'équilibre dont la densité est relativement importante; elles s'alignent selon des directions $\langle 110\rangle$ ou $\langle 010\rangle$ et des réactions analogues aux précédentes peuvent être envisagées pour rendre compte de ces directions (Fig. 5) [39]. Le déplacement du réseau intrinsèque et le contraste triple caractéristique des dislocations coin appuient, à nouveau, l'existence d'un vecteur de Burgers effectif $b_{e}$ perpendiculaire au plan du joint ou, du moins, ayant une composante normale à ce plan importante.

- Pour les joints à grande désorientation proche d'une position de coïncidence telle $\Sigma=5$, les dislocations extrinsèques ont statistiquement une orientation parallèle à $\langle 310\rangle$, c'est-à-dire à un des réseaux de dislocations secondaires. L'analyse de leur configuration la plus probable permet d'envisager la dissociation du vecteur de Burgers de la matrice en vecteurs de base du réseau D.S.C. [39] ; par exemple :

$$
\begin{gathered}
a / 2[10 \overline{1}]=a / 10[310]+a / 10[2 \overline{15}] . \\
\mathbf{b}_{1} \text { ou } \mathbf{b}_{2} \quad \mathbf{b}_{3}
\end{gathered}
$$

De même que dans les joints à faible désorientation, un vecteur de Burgers effectif $\mathbf{b}_{\mathrm{e}}=a / 2$ [001] explique le contraste et le déplacement du réseau d'équilibre.

- Citons enfin que dans les joints de torsion décrits par le modèle du Plane Matching, des dislocations extrinsèques ont été révélées avec leur vecteur de Burgers égal à $\mathbf{b}_{3}=a / 2$ [001] $=\mathbf{b}_{\mathbf{e}}$ [49].

Il y a donc un résultat commun à tous les joints de torsion [001] : quelles que soient les réactions de la dislocation de matrice dans le joint de grains, les composantes dont les vecteurs de Burgers sont dans le plan du joint sont facilement éliminées car elles sont glissiles, seules sont stabilisées les dislocations extrinsèques à caractère coin, de vecteur de Burgers effectif $\mathbf{b}_{\mathrm{e}}=a / 2$ [001] ou peu incliné sur cette direction [39].

Signalons également que dans les joints de torsion [110], les vecteurs de base du réseau D.S.C. ont été trouvés comme vecteurs de Burgers des E.G.B.D's pour $\Sigma=3$ et $\Sigma=9[41,45]$.

3.3.2 Joints spéciaux de flexion [001]. - Peu de résultats existent concernant les joints à faible désorientation mais, pour les joints à grand angle, des preuves remarquables de l'utilité du formalisme de Bollmann ont été apportées par les résultats suivants :

- la mise en évidence, dans un joint d'acier inoxydable $\left(\theta=50^{\circ}\right)$ de la dissociation d'une dislocation de matrice en cinq dislocations extrinsèques dont les vecteurs de Burgers sont $\mathbf{b}_{1}=a / 58$ [370] et $\mathbf{b}_{2}=a / 58$ [730] qui sont bien les vecteurs de base du réseau D.S.C. correspondant à $\Sigma=29\left(\theta=46^{\circ} 4\right)$ [42] ;

- la détermination, dans de l'aluminium, de dislocations isolées du joint de grains, dont les vecteurs de Burgers sont des vecteurs du réseau D.S.C. pour $\Sigma=41: \mathbf{b}_{1}=a / 82[01 \overline{9}]$ ou $\mathbf{b}_{3}=a / 82[41 \overline{45}][43] ;$ les réactions mises en jeu ont été analysées en détail récemment [44];

- des joints de flexion type C.A.D. (ou P.M.) révèlent la présence de dislocations extrinsèques de vecteur $\mathbf{b}_{3}$ [49];

- enfin, des études sur des joints de flexion [110] vont également à l'appui de réactions qui respectent le modèle de Bollmann $\left({ }^{6}\right)$.

Cependant, comme dans le cas des dislocations intrinsèques, rien n'exclut la possibilité que la première dissociation en vecteurs du réseau D.S.C. soit suivie par une dissociation en dislocations partielles; c'est ce qu'observe Pond dans un joint de macle $\{112\}$ $\Sigma=3[10]$ :

$$
\begin{aligned}
& a / 2[101]=a / 6[1 \overline{2} 1]+a / 3[111] \\
& \text { b } \quad b_{2} \quad b_{1} \\
& a / 3[111]=a / 9[111]+2 a / 9[111] \text {. } \\
& \mathbf{b}_{1} \quad b_{\text {partielles }}
\end{aligned}
$$

Contrairement aux joints de torsion, les joints de flexion peuvent contenir des dislocations extrinsèques stables ayant des vecteurs de Burgers égaux à $\mathbf{b}_{1}$ ou $\mathbf{b}_{2}$ du réseau D.S.C. puisque ces dernières ont alors un caractère sessile. Le contraste de ces E.G.B.D's supérieur à celui des dislocations intrinsèques a permis de confirmer les vecteurs de base de réseaux D.S.C. associés à des positions de coïncidence relativement faibles ( $\Sigma$ élevés) pour lesquelles le réseau d'équilibre n'est pas visible.

3.3.3 Joints généraux. - Les résultats obtenus sur les joints généraux, en apparente contradiction avec ceux obtenus sur les joints spéciaux, peuvent être classés en deux catégories :

a) Les dislocations conservent les vecteurs de Burgers possibles de la matrice.

- Ishida et Brown [93] ont trouvé dans du fer et des alliages fer-manganèse trois types de vecteurs de Burgers des dislocations extrinsèques, $a / 2\langle 111\rangle$, $a<100\rangle$ et $a\langle 110\rangle$ dans des proportions analogues à celles qu'ils ont dans les grains; cependant, dans ces travaux, les joints de grains ne sont pas analysés cristallographiquement et les vecteurs de Burgers sont déterminés en utilisant uniquement les règles d'extinction (cf. § 4.1).

- Plus récemment, en simulant le profil du contraste des dislocations et en le comparant au contraste expérimental, des travaux ont permis de déterminer deux types de vecteurs de Burgers dans des joints de grains de cuivre polycristallin : $b=a\langle 100\rangle$ et $b=a / 2\langle 110\rangle$ [38]. Dans ce cas, les désorientations et les plans des joints ont été calculés et semblent bien, a priori, quelconques; cependant, il est intéressant de noter que les joints observés présentent une pério-

$\left({ }^{6}\right)$ Très récemment Clark et Smith ont observé et analysé des dislocations extrinsèques dans des joints plus complexes [92]. 
dicité non analysée mais qui n'est pas un phénomène de Moiré ; on peut se demander s'il ne s'agit pas de joints du type Plane Matching et si les vecteurs réels des dislocations extrinsèques ne correspondent pas à des vecteurs $\mathbf{b}_{3}$ de réseaux D.S.C. Les auteurs signalent, en effet, que la méthode utilisée permet la détermination des directions des vecteurs de Burgers mais pas de leur grandeur [38].

b) Les dislocations du grain se dispersent uniformément dans le joint en modifiant sa structure intrinsèque [54]. Ce modèle peut formellement être vu comme équivalent à une dissociation en un nombre discret de dislocations possédant de très petits vecteurs de Burgers [49]. Pumphrey et Gleiter réfutent cette analogie en arguant du fait que, dans une étude statistique de plusieurs joints d'un polycristal, certains vecteurs $\mathbf{b}_{3}$ de taille comparable aux vecteurs de la matrice devraient être produits lors de la dissociation et permettre ainsi la visualisation de quelques dislocations $[51,52]$.

Très récemment, des travaux semblent appuyer l'hypothèse de la dispersion dans les joints fortement désorientés généraux en ne détectant la présence de dislocations extrinsèques que dans des joints plus ou moins spéciaux observés dans des polycristaux d'aluminium [94, 99]; les auteurs expliquent leur résultat par l'idée que le processus de dispersion intervient plus difficilement dans un joint spécial $[52,54]$.

3.3.4 Conclusions. - Malgré les contradictions évoquées entre joints spéciaux et joints généraux, nous pensons pouvoir répondre par l'affirmative au problème posé au début de ce chapitre : y a-t-il ou non réaction des dislocations de matrice avec le joint selon des processus en accord avec le formalisme de Bollmann ? En effet :

- les vecteurs de Burgers des dislocations isolées dans des joints à faible désorientation sont tous des vecteurs de Burgers du cristal;

- des vecteurs de base de différents réseaux D.S.C. ont pu être déterminés dans des joints de flexion, même lorsque la coïncidence est relativement faible ( $\Sigma$ élevé) et la structure intrinsèque non visible; - tous les joints décrits par le modèle du C.A.D. (P.M.), ou qui peuvent être vraisemblablement assimilés à ce type car présentant une périodicité structurale, contiennent des dislocations extrinsèques dont le vecteur de Burgers est (ou peut être) le vecteur $\mathbf{b}_{3}$ du réseau D.S.C. associé ;

- la détection de vecteurs de Burgers correspondant à des dislocations partielles peut s'interpréter par des réactions postérieures à l'incorporation de la dislocation de matrice dans le joint, celle-ci donnant toujours lieu à des vecteurs du réseau D.S.C. ;

- l'équivalence des modèles de dispersion et de dissociation en vecteurs infiniment petits d'un réseau D.S.C. $(\Sigma \rightarrow \infty)$ ne peut être totalement rejetée par le fait qu'aucune dislocation n'est visible dans les joints généraux; en effet, si les axes de rotation des joints au hasard dans un polycristal sont quelconques, il se peut qu'aucun vecteur $\mathbf{b}_{3}$ n'ait une taille suffisante pour qu'un contraste associé soit détectable en microscopie électronique.

3.4 LES JOINTS «AU HASARD » DANS UN POLYCRISTAL. - Du point de vue conséquences sur les propriétés des matériaux, la détermination des proportions respectives de joints spéciaux et généraux dans un polycristal est une question fondamentale. Elle peut être abordée théoriquement en considérant le polycristal formé de grains orientés au hasard mais, en réalité, les grains présentent le plus souvent une orientation préférentielle, ce qui nécessite une statistique basée sur l'observation.

La question qu'on peut formuler en terme de texture des joints demande au préalable que soit bien définie la signification physique de l'ordre intercristallin, entre autres, la nécessité ou non de détecter un phénomène périodique ; précisons : si on considère les résultats de Pond concernant le joint de flexion [001] $\Sigma=41[43,44]$, la mise en évidence de dislocations extrinsèques possédant des vecteurs de Burgers du réseau D.S.C. permet-elle d'évoquer un certain ordre physique dans l'interface, même si cet ordre n'est pas révélé par une structure intrinsèque, dans la mesure où il intervient dans les réactions d'interaction dislocations de matrice-joint de grains ?

On peut envisager deux conditions pour définir un ordre physique :

- la détection d'une structure intrinsèque soit bidimensionnelle (joints du type C.S.L.), soit unidimensionnelle (joints du type P.M.);

- la détection de dislocations extrinsèques ayant, au moins, un vecteur de Burgers du réseau D.S.C. (condition qui nécessite un état hors d'équilibre du joint).

3.4.1 «Texture des joints » basés sur leur structure intrinsèque. - Notons que l'essentiel des travaux connus, tant théoriques qu'expérimentaux, repose sur cette base.

a) Critère de Spécialité et statistique théorique. Le critère de Spécialité est défini dans un polycristal sans orientation préférentielle comme la déviation maximale à une position de coïncidence :

$$
\rho=b / d_{\min }
$$

où $d_{\min }$ est la distance minimale entre les dislocations qui doit être de l'ordre de la périodicité « $p$ » du joint.

Comme $p$ varie, pour un même $\Sigma$, avec le plan du joint, il est impossible de relier $\rho$ à la coïncidence. Warrington et Grimmer [71] considèrent que la principale valeur de $p$ varie comme $\Sigma^{1 / 3}$ et que la principale valeur de $b$ varie comme $\Sigma^{-1 / 3}$, dans ce cas :

$$
\rho=\rho_{0} \Sigma^{-2 / 3}
$$

où $\rho_{0}$ est une constante correspondant à $\Sigma=1$.

Dans le cas particulier d'un axe de torsion [100], $p$ varie comme $\Sigma^{1 / 2}, b$ varie comme $\Sigma^{-1 / 2}, \rho_{0}=8^{\circ}$ 
et $\rho=\rho_{0} \Sigma^{-1}$. Les critères de spécialité lorsque $\Sigma$ augmente $(\Sigma=1,5,13,17,25)$ sont respectivement $\rho=8^{\circ}, 1^{\circ} 6,0^{\circ} 6,0^{\circ} 5,0^{\circ} 3$ et correspondent aux observations de Schober et Balluffi (Fig. 4) [29].

$\mathrm{Du}$ point de vue statistique, en 1966, Brandon, en tenant compte uniquement de la périodicité du joint ( $p$ proportionnel à $\Sigma^{1 / 2}$ ) surestimait la proportion de joints du type C.S.L. en avançant le chiffre de $42 \%$ [27]. Des estimations plus récentes de Warrington, appuyées sur les mêmes hypothèses ( $p$ fonction de $\Sigma^{1 / 2}$ et $\left.\rho_{0}=15^{\circ}\right)$ donnent les résultats suivants : $9 \%$ de joints sont voisins d'une position de coïncidence $\Sigma=3$ à 25 et $2 \%$ sont voisins de $\Sigma=1$ [17]. Ces proportions diminuent si l'on prend le critère plus général $\rho=\rho_{0} \Sigma^{-1 / 3}$.

En ce qui concerne les joints du type P.M. ou C.A.D., la même approche théorique aboutit à $60 \%$ dans les polycristaux C.F.C. dont la moitié environ du type $\{111\}$ [17].

b) Statistique expérimentale. - Si on tient compte des probabilités d'observation, on peut s'attendre à détecter, en microscopie électronique, sur des lames polycristallines orientées au hasard des proportions de joints présentant une structure intrinsèque considérablement inférieures à celles déterminées précédemment ; $5 \%$ au lieu de $60 \%$ pour les joints type P.M. et moins de $1 \%$ pour les joints type C.S.L. Ces prévisions sont en accord avec les résultats de Ishida et al. sur l'alliage fer-manganèse [95] et, plus récemment, de Pumphrey sur des aciers inoxydables [96].

Mais dans la plupart des cas, les polycristaux présentent des textures et une petite déviation de la distribution au hasard peut donner un grand nombre de joints P.M. Dans du tungstène observé en F.I.M. et présentant une forte texture de fibre $\langle 110\rangle$, la plupart des joints ont été déterminés comme étant du type P.M. [97]. La proportion de joints spéciaux dans un acier inoxydable peut, dans certaines conditions d'obtention, atteindre $78 \%$ [98]. Compte tenu de leur faible énergie de faute d'empilement, certains métaux C.F.C. peuvent présenter $50 \%$ de macles $(\Sigma=3)$.

Enfin, l'observation récente de joints possédant une périodicité pour des écarts de flexion autour d'un axe secondaire allant jusqu'à $1^{\circ}$ augmente considérablement la probabilité de trouver des joints spéciaux du type P.M. dans un matériau (Fig. 28) [87].

Dans la pratique, le pourcentage de joints spéciaux va dépendre de nombreux facteurs, entre autres, de leur formation (solidification, recristallisation primaire ou secondaire...) et de la pureté du matériau.

3.4.2 «Texture des joints » basée sur leur structure extrinsèque. - Nous pensons qu'il est possible actuellement d'étendre le concept de spécialité des joints de grains. En effet, des travaux récents montrent que les dislocations de la matrice se dispersent lors de leur intégration dans un joint général, cette dispersion étant un phénomène activé thermiquement [52, 92, 94]. La non-observation de dislocations isolées

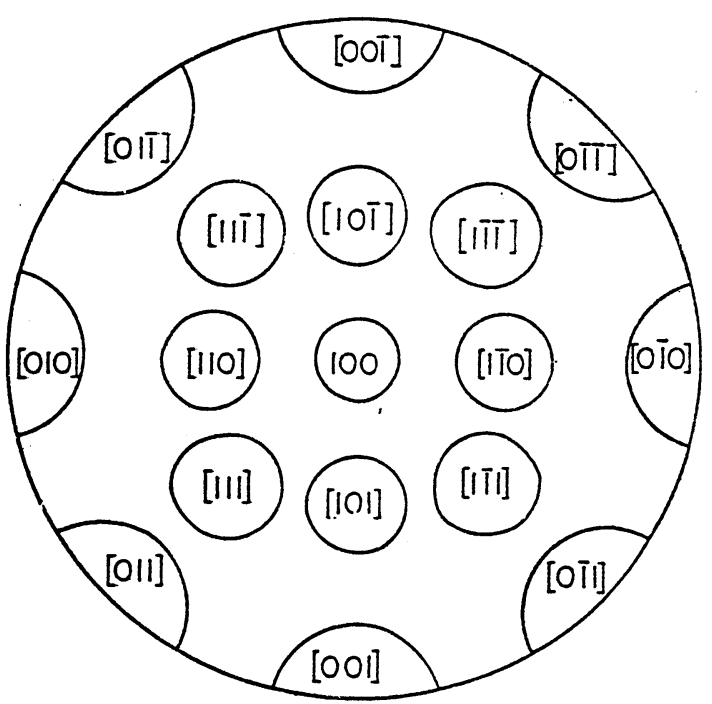

Fig. 28. - Projection stéréographique des domaines de désorientation angulaire (à l'intérieur des petits cercles) pour lesquels des dislocations intrinsèques de vecteurs $\mathbf{b}_{3}$ peuvent être stabilisées dans des joints du type Plane Matching [87].

[Standard stereographic projection of angular misorientation space (within smaly circles) over which discrete $\mathbf{b}_{3}$ intrinsic dislocations may be stable in Plane Matching boundaries [87].]

dans un joint d'un échantillon ayant subi une contrainte plastique peut donc être un critère de son caractère général. Réciproquement, la stabilité de dislocations extrinsèques même, après un recuit qui produit un équilibre local, peut permettre de conférer aux joints un certain caractère spécial comme le suggèrent des travaux récents $[94,99]$.

Cependant, si ce concept élargi de spécialité apparaît intéressant du point de vue des joints réels et de leurs propriétés, il est difficilement exploitable actuellement pour diverses raisons :

- difficulté d'identifier les vecteurs de Burgers des dislocations de joints de grains (cf. $\S 4.1$ );

- interaction possible, mal connue, des dislocations extrinsèques avec les atomes d'impuretés ségrégées ou les précipités intergranulaires [100, 101, 174] ;

- influence du mode de formation des dislocations extrinsèques, c'est-à-dire de l'histoire thermo-mécaniqué de l'échantillon.

3.4.3 Conclusion. - Etudier les joints au hasard dans un polycristal, c'est-à-dire expliciter la notion de texture des joints de grains, apparaît actuellement comme un problème essentiel en métallurgie mais extrêmement difficile à aborder; il est, en effet, subordonné à une question qui n'admet pas jusqu'à présent de réponse rigoureuse : Qu'est-ce qu'un joint spécial ?

Formellement, il convient de déterminer jusqu'à quel point une structure de joint de grain peut être considérée comme ordonnée lorsque la désorientation de l'interface s'écarte de celle correspondant à une réelle structure périodique. 
4. Microscopie électronique des joints de grains. L'étude en microscopie électronique des joints de grains se développe dans deux directions étroitement liées :

- l'analyse des contrastes des défauts propres au joint,

- la géométrie des défauts (ligne de dislocation, vecteur de Burgers) qui demande au préalable la détermination des paramètres cristallographiques du joint de grains (axe et angle de désorientation des grains voisins, plan du joint).

L'approche du microscopiste est donc très différente selon qu'il observe des dislocations intrinsèques ou des dislocations extrinsèques, des joints spéciaux de bicristaux préorientés ou des joints réels de polycristaux. L'étude géométrique des dislocations intrinsèques est relativement aisée car le formalisme de Bollmann permet de prévoir leurs caractéristiques, par contre, il est difficile d'analyser les contrastes associés à ces dislocations car leurs champs de contrainte sont très localisés dans l'interface [54, 102].

Les paramètres cristallographiques des dislocations extrinsèques dépendent des réactions d'interaction des dislocations de matrice avec le joint et demandent donc pour être déterminés une connaissance précise de celui-ci. On conçoit alors une démarche très différente selon qu'on considère des dislocations extrinsèques dans un joint de bicristal bien défini ou dans un joint au hasard de polycristal ; par ailleurs, les règles d'extinction utilisées pour déterminer les vecteurs de Burgers des dislocations de matrice sont rarement opérantes dans le cas des joints de grains, il est nécessaire de s'appuyer sur l'analyse des intensités transmise et diffractée à la sortie de la lame; ces intensités peuvent être calculées car les champs de contrainte des dislocations extrinsèques s'étendent dans les grains voisins mais les résultats restent très controversés compte tenu, entre autres, des différentes hypothèses sur l'élasticité du milieu.

Nous distinguerons donc l'étude microscopique des dislocations isolées de celle des réseaux de dislocations; mais auparavant, quel que soit le type de dislocation observé, il convient de souligner les difficultés inhérentes à cette étude. En' effet, les contrastes dus aux dislocations de joints de grains et à la désorientation des deux cristaux adjacents résultent, en général, de l'excitation de plusieurs réflexions de Bragg dans chacun des grains entraînant des phénomènes de diffraction et de double diffraction complexes [84]. On tentera donc de se placer dans des conditions relativement simples de diffraction que l'on peut résumer en trois cas (Fig. 29) [34] :

- condition d'observation en deux ondes : un seul des cristaux est fortement excité, l'autre est considéré comme une couche amorphe; les faisceaux intenses à la sortie de la lame seront donc, soit $\mathrm{O}_{1} \mathrm{O}_{2}$ et $\mathrm{G}_{1} \mathrm{O}_{2}$ si le cristal 1 diffracte seul, soit $\mathrm{O}_{1} \mathrm{O}_{2}$ et $\mathrm{O}_{1} \mathrm{G}_{2}$ si le cristal 2 diffracte seul;

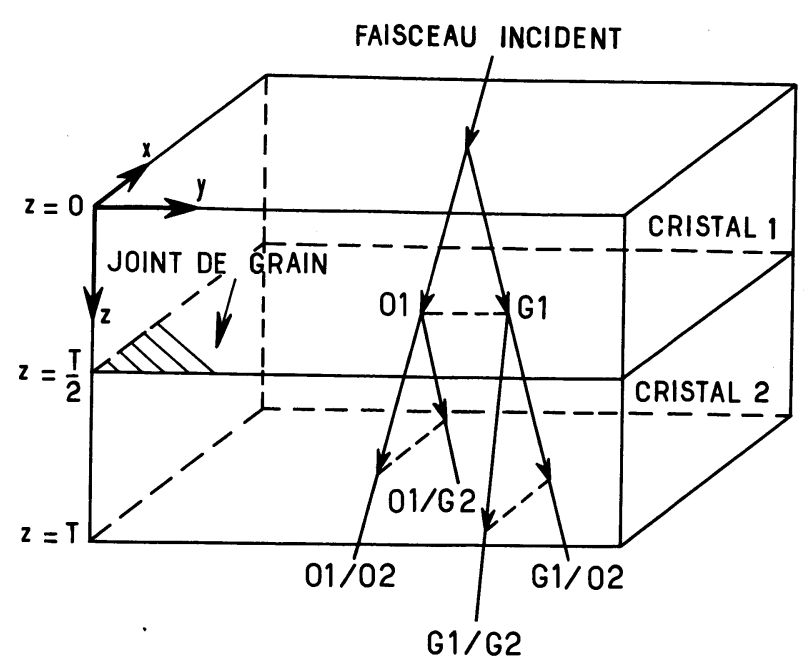

Fig. 29. - Géométrie de la diffraction dans un bicristal [34].

[Geometry of diffraction in bicrystal specimen [34].]

- condition d'observation en trois ondes obtenue si les deux cristaux sont en position de Bragg par rapport au faisceau incident $\left(\mathrm{O}_{1} \mathrm{O}_{2}, \mathrm{G}_{1} \mathrm{O}_{2}\right.$ et $\mathrm{O}_{1} \mathrm{G}_{2}$ intenses) ou si le cristal 2 est en position de Bragg par rapport au faisceau diffracté dans le cristal 1 $\left(\mathrm{O}_{1} \mathrm{O}_{2}, \mathrm{G}_{1} \mathrm{O}_{2}\right.$ et $\mathrm{G}_{1} \mathrm{G}_{2}$ intenses $)$;

- condition en quatre ondes dans le cristal 2; tous les faisceaux ont une intensité non négligeable à la sortie de la lame.

L'intensité de chacun des faisceaux peut être calculée en considérant que les quatre ondes dans le cristal 2 peuvent être couplées dynamiquement seulement par paires $\left(\mathrm{O}_{1} \mathrm{O}_{2}\right.$ avec $\mathrm{O}_{1} \mathrm{G}_{2}$ et $\mathrm{G}_{1} \mathrm{O}_{2}$ avec $\mathrm{G}_{1} \mathrm{G}_{2}$ ).

En intégrant les équations de Howie et Whelan [84] dans le cristal 1, on obtient les amplitudes des faisceaux avant leur interaction avec le cristal 2. Chacun est alors considéré comme un faisceau incident pour le cristal inférieur et les mêmes intégrations permettent d'obtenir les amplitudes de $\mathrm{O}_{1} \mathrm{O}_{2}$ et $\mathrm{O}_{1} \mathrm{G}_{2}$ d'une part et de $G_{1} O_{2}$ et $G_{1} G_{2}$ d'autre part.

Dans de nombreuses conditions, les quatre faisceaux divergent suffisamment pour qu'ils puissent être séparés par le diaphragme d'objectif et les contrastes obtenus expérimentalement peuvent être comparés aux calculs précédents. Cependant $G_{1} O_{2}$ et $O_{1} G_{2}$ sont, dans certains cas, extrêmement voisins, le diaphragme de l'objectif ne permet pas de les séparer, ils donnent lieu à des franges d'interférence de Moiré qui, dans certaines conditions, se présentent d'une manière analogue aux réseaux de dislocations intrinsèques; l'image consiste alors en une superposition de divers types de contrastes qu'il convient d'analyser avec précaution.

4.1 ETUde D'Une Dislocation ISOlÉe (EXTRINSÈQuE). - Bien que les réactions des dislocations de matrice avec un joint ne semblent pas quelconques, le plus souvent l'étude géométrique des dislocations 
extrinsèques nécessite la comparaison du contraste observé et du contraste calculé. Ce contraste dû à la déformation élastique autour de la dislocation va dépendre d'un grand nombre de facteurs liés aux paramètres intervenant dans les équations d'intégration et qu'on peut distinguer en deux catégories :

a) ceux qui dépendent de la lame, c'est-à-dire qui influent sur $\mathbf{z}$ et $\mathbf{R}$ ( $\mathbf{R}$ étant le vecteur déplacement atomique qui décrit la déformation due au défaut situé à la profondeur z). Citons, entre autres :

- la position du plan du joint par rapport à la surface de la lame. On conçoit qu'il sera plus aisé d'observer et surtout de calculer le contraste associé à une dislocation située dans un plan parallèle à la surface de la lame et perpendiculaire au faisceau d'électrons car, dans ce cas, il n'y a aucune variation d'épaisseur dans chacun des cristaux et les bornes d'intégration sont parfaitement définies;

- les paramètres cristallographiques du joint (angle et axe de rotation - plan du joint) qui permettent de prévoir les vecteurs de Burgers des dislocations. Ceci distingue très nettement les joints préorientés des joints réels ;

- la géométrie de l'orientation du défaut (caractère coin ou vis de la dislocation);

- l'hypothèse utilisée pour calculer le champ de contrainte ; en particulier, dans quelle mesure peut-on s'appuyer sur la théorie de l'élasticité linéaire pour les milieux continus isotropes ?

b) ceux qui dépendent des conditions d'observation :

- vecteur de diffraction $\mathbf{g}$,

- écart à la position de Bragg $\mathbf{s}$,

- étude en deux ou trois ondes.

Ces facteurs sont interdépendants. La comparaison des contrastes observé et calculé est valable :

- si les conditions d'obtention expérimentale sont bien définies et peuvent être réalisées;

- si l'approximation utilisée dans le calcul décrit de façon adéquate ces conditions;

- si les images présentent un contraste suffisamment fort pour être distingué dư fond continu.

4.1.1 Contraste d'une dislocation dans les conditions de deux ondes. - Cette condition est facilement réalisée lorsque les deux cristaux sont fortement désorientés ; un seul cristal est en position de diffraction, les interactions avec le second cristal sont négligées ainsi que les contraintes dans l'interface, ce qui signifie que l'on considère les dislocations à la surface libre voisine du joint dans le cristal diffractant. Il semble, à première vue, contradictoire d'utiliser dans ce cas un vecteur déplacement $\mathbf{R}$ dû à un champ de contrainte calculé avec l'approximation des milieux élastiques continus isotropes. Cependant, les analyses et les observations effectuées avec ces hypothèses ont donné des résultats valables aussi bien pour un joint parallèle à la surface de la lame d'un bicristal que pour un joint incliné dans un polycristal $[34,36$, $38,103,104,105,106]$.

a) Etude d'une dislocation dans un joint parallèle à la surface de la lame. - Selon le caractère coin ou vis de la dislocation, deux conditions de contraste sont à considérer :

Premier cas : b est contenu dans le plan du joint.

Soit $\mathbf{g}$ le vecteur de diffraction et $\mathbf{u}$ un vecteur unitaire de la ligne de dislocation, le produit g.b $\wedge \mathbf{u}=0$, les trois vecteurs étant coplanaires : les dislocations coin sont en condition d'extinction, seules, les dislocations vis peuvent être visualisées à condition que $\mathbf{g} \cdot \mathbf{b} \neq 0$. Connaissant les divers paramètres qui interviennent dans les équations de Howie et Whelan, on peut obtenir théoriquement le profil des intensités transmise et diffractée en fonction de la distance à la ligne de dislocation selon que le cristal 1 ou 2 est excité [34] (Fig. 30). On trouve, bien entendu, le type de contraste fortement asymétrique caractéristique des dislocations vis proches de la surface d'un monocristal. Les profils de contraste sont inversés lorsque g.b change de signe. Pratiquement, on peut résumer les observations par le schéma de la figure 31.

Deuxième cas : b est perpendiculaire au plan du joint, $\mathbf{g} \cdot \mathbf{b}=0$. On ne peut pas observer de dislocations vis. Le contraste des dislocations coin dépend du produit mixte $\mathbf{g} \cdot \mathbf{b} \wedge \mathbf{u}$; en particulier, on a intérêt à se placer dans des conditions de diffraction telles que le vecteur $\mathbf{g}$ a une forte composante parallèle à $\mathbf{b} \wedge \mathbf{u}$; dans ce cas, les calculs laissent prévoir que la dislocation observée en fond clair et en fond noir présentera un contraste symétrique (contraste triple) (Fig. 32). Comme précédemment, les profils sont inversés avec le changement de signe de $\mathbf{g . b} \wedge \mathbf{u}$. Les observations sont résumées dans le schéma de la figure 33.

b) Etude d'une dislocation dans un joint incliné par rapport à la surface de la lame. - Bien que les premières études du contraste associé à une dislocation inclinée étaient basées sur la considération d'une dislocation parallèle à la surface de la lame et à midistance dans celle-ci, la forme du contraste observé était en accord avec les prévisions théoriques [103]. Cette constatation peut être justifiée par les calculs ultérieurs qui, tenant compte de l'inclinaison de la dislocation, ont montré que les profils d'intensité préservent leur forme le long de la ligne de dislocation lorsque l'écart à la position de Bragg est nul [105]. Cette règle est très importante pour la distinction d'une dislocation située dans le joint d'une dislocation de matrice voisine du joint : en effet, la dislocation extrinsèque ne présentera jamais le contraste oscillant caractéristique de la dislocation de matrice voisine de la surface du cristal diffractant.

Notons que les règles de contraste telles qu'elles se dégagent des figures 31 et 33 sont conservées :

- contraste inversé en fond clair et en fond noir si le grain supérieur diffracte; 


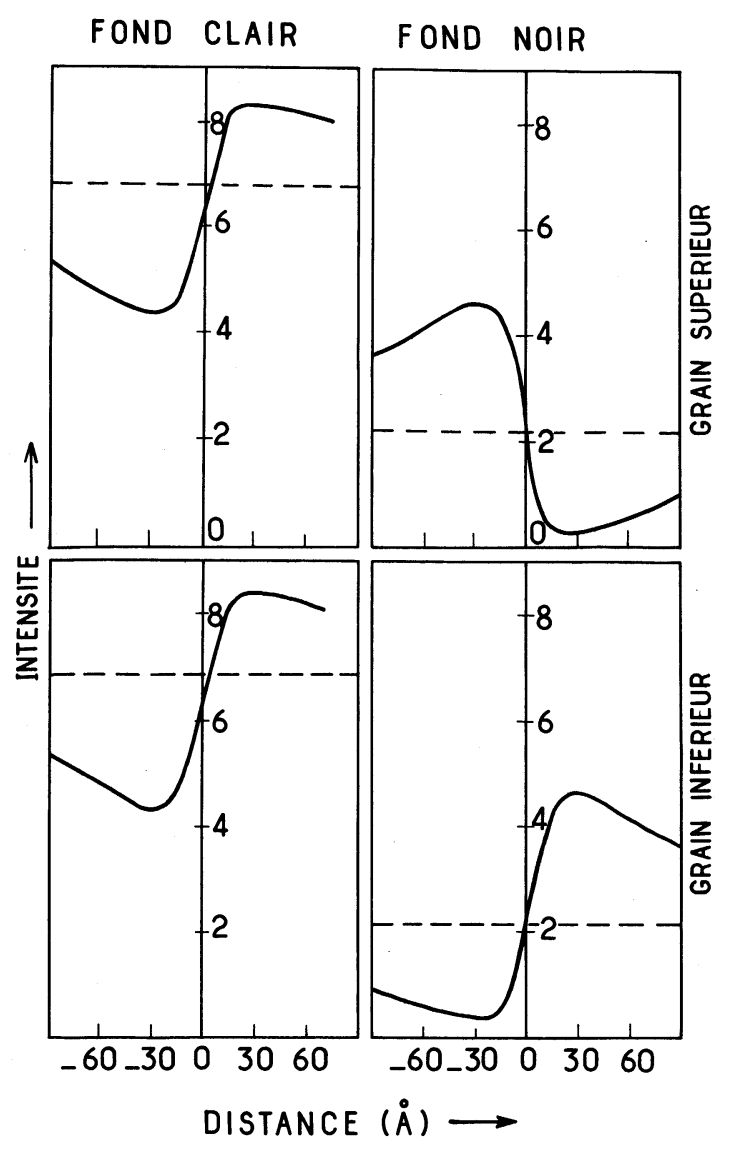

Fig. 30. - Profils de contraste d'une dislocation intergranulaire de caractère vis. Les intensités sont calculées, pour l'or, en fond clair et en fond noir dans les conditions de deux ondes avec $s=0$ pour le faisceau fortement excité et $s=0,1$ pour les faisceaux faiblement excités (épaisseur du bicristal $=800 \AA, g=200$, g.b $=1$ ). Le joint est parallèle aux surfaces d'entrée et de sortie de la lame et situé en son milieu. En pointillé, l'intensité du fond continu à grande distance de la ligne de dislocation [34].

[Calculated diffraction contrast intensity profiles for screw GBD with one crystal strongly excited in two-beam situation. Intensities in bright field and in dark field were calculated for gold with foil thickness $=800 \AA, \mathbf{g} \cdot \mathbf{b}=1, \mathbf{g}=200$. In each case strongly excited reflection has $s=0$, and weakly excited reflections have $s=0.1$. Background intensity far from dislocation indicated by dashed line [34].]

\begin{tabular}{|c|c|c|c|c|c|c|c|}
\hline \multicolumn{4}{|c|}{ grain supérieur } & \multicolumn{4}{|c|}{ grain inférieur } \\
\hline$F C$ & $F C$ & $F N$ & $F N$ & $F C$ & $F C$ & $F N$ & $F N$ \\
\hline$+g$ & $-g$ & $+g$ & $-g$ & $+g$ & $-g$ & $+g$ & $-g$ \\
\hline $\mathbb{\Delta}$ & $\square$ & 미 & $\square$ & $\mathbb{N}$ & $\square$ & (1) & 뭄 \\
\hline
\end{tabular}

Fig. 31. - Schéma résumant les changements de contraste d'une dislocation extrinsèque $V i$ en fonction des conditions de diffraction.

[Dependence of type of contrast of a screw extrinsic dislocation on diffraction conditions.]

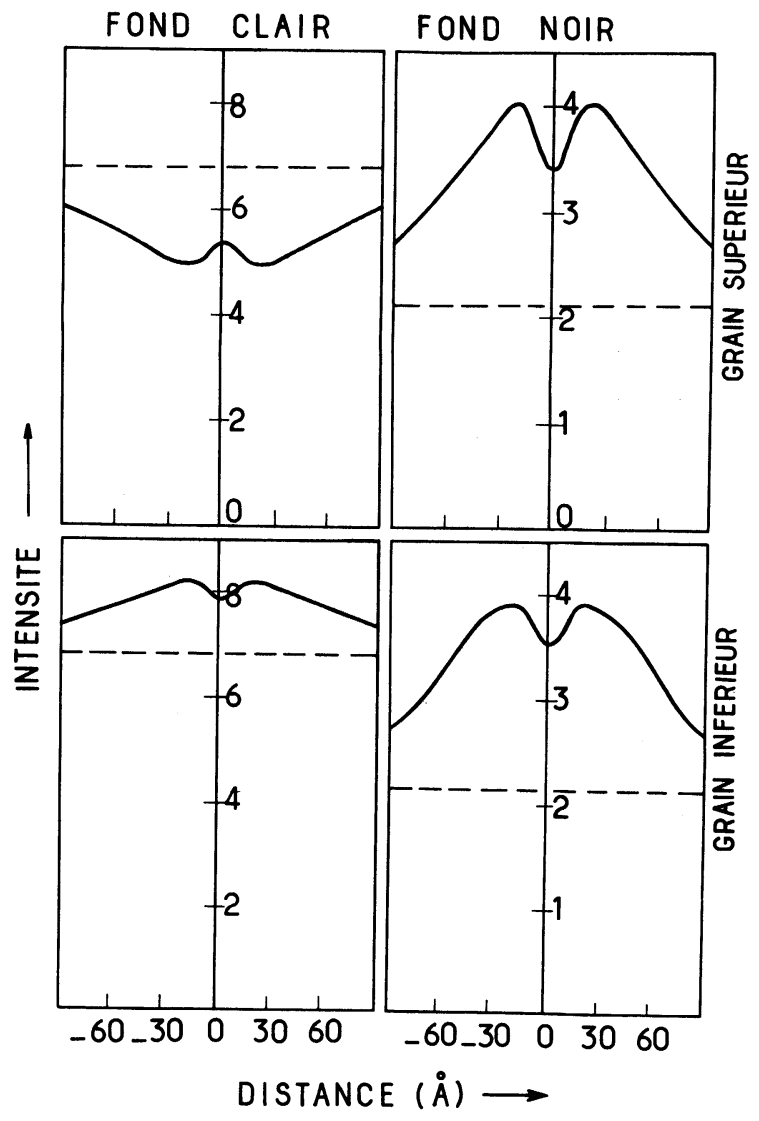

Fig. 32. - Profils de contraste d'une dislocation intergranulaire de caractère coin. Les intensités sont calculées, pour l'or, en fond clair et en fond noir dans les conditions de deux ondes (épaisseur de lame $=800 \AA, \mathbf{g} \cdot \mathbf{b}=0, m=1 / 8 \mathbf{g} \cdot \mathbf{b} \times \mathbf{u}=+0,102$ ). Le joint est parallèle aux surfaces d'entrée et de sortie de la lame et situé en son milieu. En pointillé, l'intensité du fond continu à grande distance de la ligne de dislocation [34].

[Calculated diffraction contrast intensity profiles for edge GBD with one crystal strongly excited in two beam situation (gold, $T=800 \AA, \mathbf{g} . \mathbf{b}=0, m=1 / 8 \mathrm{~g} . \mathbf{b} \times \mathbf{u}=+0.102$ ). The boundary plane is parallel to the foil surfaces and situated in the middle of the foil. Background intensity far from dislocation indicated by dashed line [34].]

\begin{tabular}{|c|c|c|c|c|c|c|c|}
\hline \multicolumn{3}{|c|}{ grain supérieur } & \multicolumn{4}{|c|}{ grain inférieur } \\
\hline \hline$F C$ & $F C$ & $F N$ & $F N$ & $F C$ & $F C$ & $F N$ & $F N$ \\
$+g$ & $-g$ & $+g$ & $-g$ & $+g$ & $-g$ & $+g$ & $-g$ \\
\hline & $\square$ & $\square$ & & & & & \\
\hline
\end{tabular}

Fig. 33. - Schéma résumant les changements de contraste d'une dislocation extrinsèque Coin en fonction des conditions de diffraction.

[Dependence of type of contrast of an edge extrinsic dislocation on diffracting conditions.] 
- contraste identique en fond clair et en fond noir si le grain inférieur diffracte;

- changement de contraste quel que soit le grain diffractant si $\mathbf{g}$ ou $\mathbf{b}$ change de signe.

La visualisation d'une dislocation pour des conditions rigoureusement de deux ondes dans chaque cristal permet de la situer, avec une grande probabilité, dans l'interface. Les différentes observations qui conduisent à l'identification d'une dislocation extrinsèque ont été récemment résumées par Murr et Ventakesh bien qu'une certaine confusion entre ledge et dislocation extrinsèque existe dans la terminologie utilisée par ces auteurs [107].

L'orientation de la ligne de dislocation, en général non perpendiculaire au faisceau d'électrons (contrairement au cas (a) précédent) entraîne des variations complexes du contraste; en particulier, dans le cas d'une dislocation coin, le contraste dépend :

- de l'orientation de b par rapport au plan du joint. Les profils calculés deviennent très diffus lorsque b est contenu dans le plan du joint,

- de l'angle $\varphi$ entre $\mathbf{g}$ et la projection de la ligne de dislocation dans le plan de l'image. C'est seulement lorsque $\varphi=90^{\circ}$ que le schéma de la figure 33 peut être utilisé.

Enfin, lorsque la dislocation a un caractère mixte, les variations du contraste selon l'angle $\varphi$ sont difficiles à systématiser.

Si on choisit les conditions de diffraction en tenant compte des remarques précédentes, la comparaison des profils d'intensité calculés et observés est possible, elle a permis, entre autres, l'identification de vecteurs de Burgers dans le cuivre polycristallin [38] (Fig. 34).

c) Critique de l'observation en deux ondes. Deux restrictions importantes sont à considérer :

- l'une due à l'inadéquation de l'approximation sur la contrainte aux conditions d'observation,

- l'autre due à la difficulté de réaliser expérimentalement les conditions d'observation rigoureuses en deux ondes.

Le fait que la discontinuité du champ de contrainte introduite par le joint n'est pas prise en compte sur la base de la théorie de l'élasticité linéaire pour les milieux continus a été souligné par McDonald et Ardell [36] ; ces auteurs envisagent, au moins, deux vecteurs $\mathbf{R}$ pour décrire la déformation due à une dislocation extrinsèque, un dans chacun des cristaux adjacents. Bien entendu, le vecteur utilisé dans les équations de Howie et Whelan [84] correspondra au vecteur déplacement $\mathbf{R}$ dans le grain diffractant [108]. De plus, il semble important de le choisir dans le grain qui contenait la dislocation de matrice ayant réagi avec le joint [36].

Cette première restriction concernant la contrainte a été levée par Varin et al. [105] qui utilisent l'approximation du milieu élastique continu et isotrope en se basant sur la constatation que la symétrie de l'image

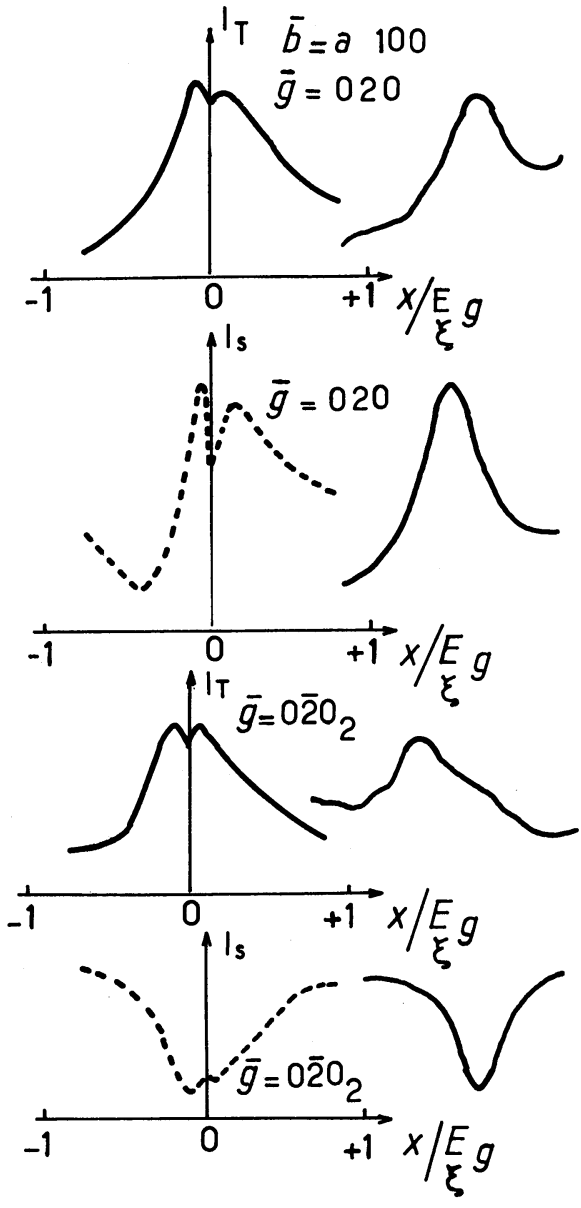

Fig. 34. - Comparaison des profils d'intensité calculé (à gauche) et mesuré (à droite) pour une dislocation extrinsèque dans du cuivre, pour deux conditions de diffraction : $\mathbf{g}=020_{1}$ et $\mathbf{g}=02 \mathrm{O}_{2}$; $I_{\mathrm{T}}$ : intensité en fond clair, $I_{\mathrm{S}}$ : intensité en fond noir [38]

[Computed (left) and measured (right) intensity profiles for an extrinsic dislocation in copper, in two diffraction conditions : $\mathbf{g}=020_{1}$ and $\mathbf{g}=0 \overline{2} 0_{2} ; I_{\mathrm{T}}$ : intensity on the bright field image, $I_{\mathrm{S}}$ : intensity on the dark field image [38].]

est invariante avec le degré d'anisotropie élastique [109].

La seconde restriction s'appuie sur l'observation que le cristal non diffractant ne se comporte pas simplement comme un milieu absorbant pour les électrons et qu'il faut tenir compte des faisceaux faiblement diffractés dans ce grain. D'autre part, les conditions de deux ondes ne peuvent pas être réalisées pour des joints à faible désorientation.

4.1.2 Observation en trois ondes ou "deux ondes" simultanées. - Les deux cristaux sont simultanément en condition de diffraction en deux ondes. A partir des mêmes hypothèses sur la déformation que précédemment, les profils d'intensité de chacun des faisceaux $\mathrm{O}_{1} \mathrm{O}_{2}, \mathrm{O}_{1} \mathrm{G}_{2}$ et $\mathrm{G}_{1} \mathrm{O}_{2}$ (Fig. 29) ont été calculés dans le cas d'une dislocation coin avec b perpendiculaire au plan du joint et parallèle au faisceau d'électrons : ils se présentent avec un contraste symétrique [34]. Très souvent, le contraste d'une dislocation 
extrinsèque se détache mal du fond continu du joint car il apparaît des franges de Moiré qui compliquent l'observation.

Par ailleurs, des images théoriques d'une dislocation ont été obtenues par simulation à l'ordinateur dans le cas de la théorie à deux ondes simultanées [35]. Les auteurs ont combiné les équations de Howie et Whelan dans chaque cristal en utilisant un vecteur déplacement qui rend compte de la contrainte associée à une dislocation située entre deux demi-espaces élastiques anisotropes [110]. Les vecteurs diffractant dans chaque grain sont différents, seul le faisceau incident est commun aux deux grains : l'observation du joint, dans ces conditions, s'effectue donc toujours en fond clair. Pour éviter le risque de faisceaux multiples dans le cristal inférieur, il est nécessaire d'utiliser les lignes de Kikuchi de telle façon que la tache de diffraction correspondant au cristal 1 soit fortement écartée de la condition de Bragg pour le cristal 2 (Fig. 35). Pour identifier un défaut dans un joint, il convient d'utiliser, au moins, trois vecteurs diffractants non coplanaires dans chaque cristal ; en fait, la comparaison des images obtenues et calculées s'effectue sur plus de six cas.

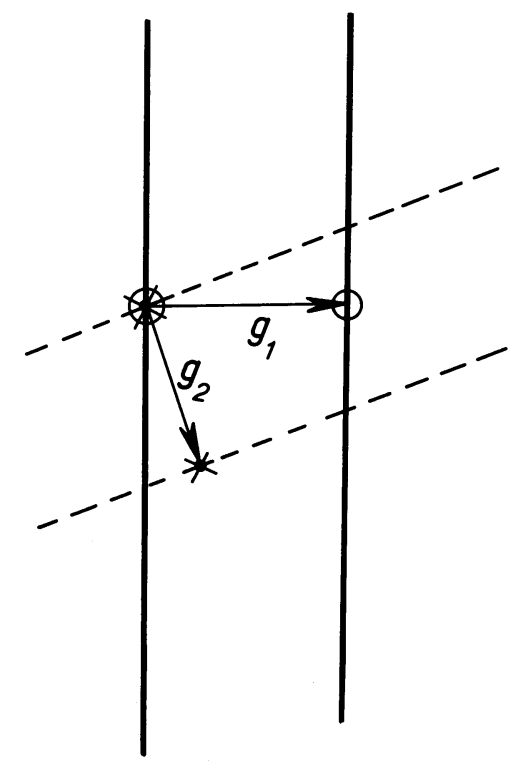

Fig. 35. - Positions respectives des lignes de Kikuchi et des taches de diffraction dans l'observation, en deux ondes simultanée.

[Respective positions of the Kikuchi lines and diffraction spots in the simultaneous two-beams condition.]

Des résultats intéressants ont été obtenus avec cette approximation mais les auteurs soulignent la difficulté, dans ce cas, à distinguer une dislocation située dans le joint d'une dislocation très voisine de celui-ci [35].

Actuellement il semble plus aisé de travailler avec l'approximation à deux ondes. En plus de l'analyse des contrastes, l'étude de la cristallographie du défaut nécessite le report de toute direction et plan dans un système de référence attaché à l'un des grains qui est mieux défini si ce grain est seul en condition de diffraction.

4.1.3 Analyse géométrique d'une dislocation extrinsèque. - Il s'agit de déterminer la ligne de dislocation et le vecteur de Burgers de cette dislocation.

a) Ligne de dislocation. - L'orientation d'une dislocation extrinsèque est relativement aisée à déterminer pour les joints spéciaux à faible ou forte désorientation quand on visualise le réseau de dislocations intrinsèques dont les caractéristiques sont connues à partir du C.S.L. [39].

Dans le cas d'un joint général, on est confronté à un problème classique en microscopie électronique. Rappelons qu'il suffit de reporter stéréographiquement la projection de la ligne de dislocation pour deux valeurs au moins de l'angle de basculement correspondant à des conditions de deux ondes. L'intcrsection de deux méridiens passant chacun par un pôle de cette projection et l'axe de zone correspondant est le pôle de la droite (Fig. 36). Bien entendu, les indices de la ligne de dislocation diffèrent dans un grain et dans

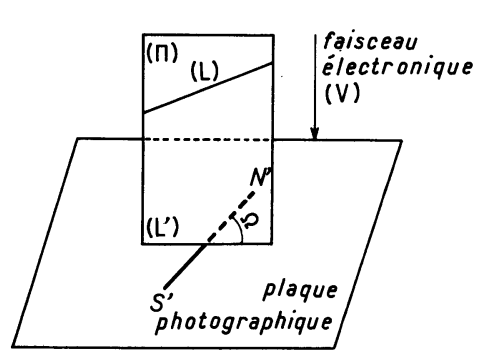

(a)

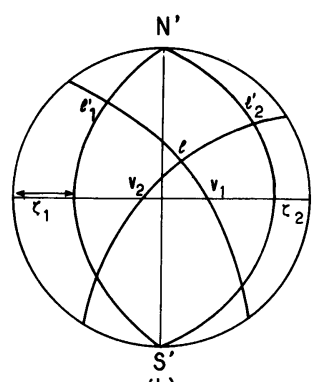

(b)
Fig. 36. - Détermination des indices d'une direction : a) Définition du plan projetant ; b) Construction du pôle de la direction sur la projection stéréographique.

[Determination of the direction indices : $a$ ) Definition of the projecting plane. b) Construction of the direction pole upon the stereographic projection.]

l'autre. Le plus souvent, cette ligne correspond à l'intersection d'un plan de glissement d'un des cristaux avec le plan du joint $[38,99,100]$; c'est ce cristal origine de la dislocation de matrice qui sera choisi comme référentiel. Le plan du joint peut être déterminé en superposant la projection de sa trace et l'axe de basculement et en minimisant sa largeur projetée (Fig. 37); cette méthode est surtout valable lorsque le plan du joint est fortement incliné dans la lame, la précision est de l'ordre de $4^{\circ}$.

b) Vecteur de Burgers. - L'identification de $b$ nécessite très souvent la comparaison de plusieurs images de la dislocation obtenues pour différentes réflexions de Bragg avec les images théoriques calculées en utilisant différents vecteurs de Burgers possibles de cette dislocation. On peut :

- soit comparer le profil théorique de l'intensité transmise ou diffractée en fonction de la distance à la 

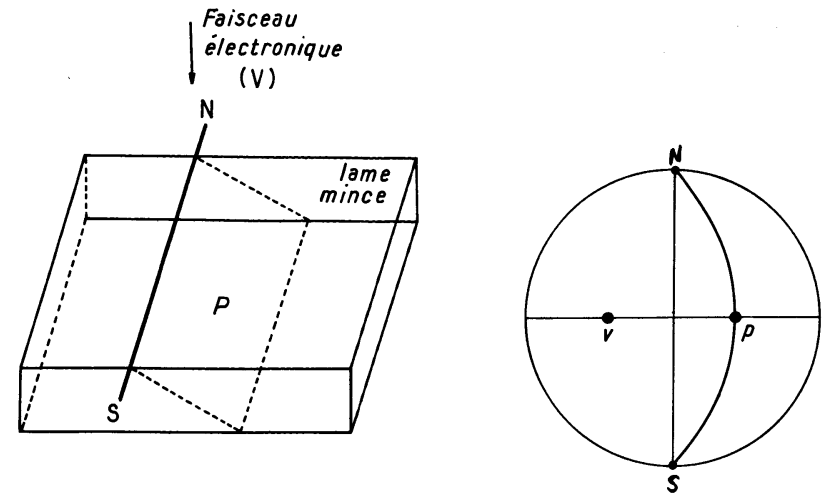

Fig. 37. - Construction du pôle $p$ d'un plan $(h k l)$

[Determination of the grain boundary plane by mean of the stereographic projection.]

ligne de dislocation avec le profil expérimental analysé par microdensitométrie $[38,105]$;

- soit simuler à l'ordinateur l'image théorique et la confronter à la micrographie observée $[35,106]$.

Mais il convient, en premier lieu, de sélectionner les diverses possibilités pour b qui dépendent des relations d'orientation entre grains et du plan du joint. Les hypothèses sont :

- b est un vecteur possible de la matrice (possible ne signifie pas effectif);

- b est un vecteur du réseau D.S.C., le plus souvent le vecteur $\mathbf{b}_{3}$ dont la longueur est bien supérieure à celles de $\mathbf{b}_{1}$ et $\mathbf{b}_{2}$.

Dans le cas d'un joint général, on n'utilisera que les vecteurs de Burgers possibles des grains voisins.

L'étude d'un joint au hasard dans un polycristal commence donc par la détermination des relations d'orientation entre les grains adjacents et du plan $\mathrm{du}$ joint afin de classer ce joint du point de vue de sa spécialité et de connaître, éventuellement, le réseau D.S.C. associé. Différentes méthodes sont proposées pour obtenir l'axe et l'angle de désorientation entre deux grains [111 à 116]. L'utilisation des lignes de Kikuchi permet une bonne précision sur l'orientation des cristaux. A partir de là, on peut soit reporter les clichés correspondant à chaque cristal sur une projection stéréographique et déterminer les axes et les angles de rotation par construction, soit calculer directement à partir des clichés la matrice qui décrit la rotation d'un cristal par rapport à l'autre, ce qui conduit à une précision bien meilleure $\left(10^{\prime}\right.$ au lieu de $2^{\circ}$ environ).

Pour la plupart des systèmes cristallins métalliques, plusieurs rotations existent pour décrire la même relation d'orientation entre deux réseaux (1 152 possibilités dans le système cubique) $[117,118]$; certains auteurs se sont donc attachés à trouver une description unique et pratique de la désorientation entre grains afin de pouvoir comparer des travaux de différentes origines $[118,119]$. La méthode des quaternions unitaires permet de déterminer les couples axe/angle de désorientation [118]; une valeur limite de l'angle de désorientation est définie pour chaque système ( $62^{\circ} 8$ pour le système cubique), l'axe étant situé dans un triangle stéréographique standard particulier de la projection stéréographique [119]. Malgré l'intérêt d'une telle normalisation, qui ne pose aucun problème dans le cas des joints généraux, on peut se demander si elle aboutit à la description la plus opérante dans le cas d'un joint spécial car elle ne tient pas compte du plan du joint. Précisons : pour un $\Sigma$ donné, si le joint présente un phénomène périodique attribuable à un réseau de dislocations intrinsèques de vecteur $\mathbf{b}_{3}$, l'axe de rotation est alors défini; mais en l'absence d'une telle périodicité, il n'est pas évident que les critères de normalisation précédents donnent la description la plus adéquate du joint, en particulier, il nous semble important de considérer la position des différents axes de rotation possibles par rapport au plan du joint. La présence de dislocations extrinsèques dont on pourrait déterminer les vecteurs de Burgers peut aider à décrire le joint en permettant d'atteindre son réseau D.S.C.; mais on se heurte à une contradiction car, le plus souvent b ne peut être déterminé qu'à partir de la connaissance de lă géométrie du joint. En fait, la présence de dislocations extrinsèques dans une interface permet plutôt de confirmer l'hypothèse retenue pour sa description.

Pratiquement on peut faire une première prospection du vecteur de Burgers en cherchant des conditions d'extinction qui ne peuvent être parfaitement remplies que dans deux cas :

- une dislocation de caractère purement vis;

- une dislocation de caractère coin avec la ligne de dislocation perpendiculaire au faisceau d'électrons.

Le plus souvent, ces conditions ne sont pas remplies et la détermination de $\mathbf{b}$, fondamentale pour comprendre les réactions des dislocations de matrice avec le joint et certaines propriétés intergranulaires, est extrêmement complexe.

Pour les joints spéciaux dont le plan est perpendiculaire au faisceau d'électrons, le réseau de dislocations intrinsèques est souvent visualisé, rappelons alors que le vecteur de Burgers d'une dislocation extrinsèque peut être déduit de la configuration de sa ligne en utilisant la procédure de Frank [40] (cf. $\S 3.3 .1)$.

4.2 ETUDE DES RÉSEAUX DE DISLOCATIONS INTRINSÈQUES. - L'interprétation des observations en microscopie électronique de dislocations intrinsèques s'appuie généralement sur la considération que les contraintes dues aux dislocations arrangées périodiquement dans l'interface s'annulent à grande distance. Le bicristal peut alors être considéré comme constitué de trois régions (Fig. 38) :

- le cristal 1 et le cristal 2 libres de contraintes, qui se déduisent l'un de l'autre par rotation;

- la région du joint où se localisent les contraintes et dont la largeur est sensiblement égale à la distance entre dislocation $d_{\mathrm{D}}$. 


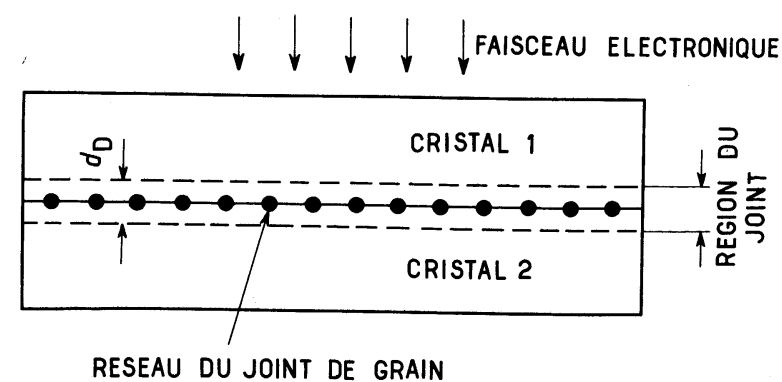

Fig. 38. - Schéma d'un bicristal montrant les régions des cristaux 1 et 2 adjacentes à la région du joint où se localisent les contraintes arrangées périodiquement.

[Schematic drawing of a bicrystal specimen showing crystal regions adjoining a regular array of intrinsic GBD's.]

Lorsque l'écart à la coïncidence augmente, $d_{\mathbf{D}}$ diminue, le volume du matériau perturbé par le joint est moins important et l'intensité de diffusion des électrons dans cette région diminue. Il apparaît, $a$ priori, difficile de résoudre des réseaux de dislocations si $d_{\mathrm{D}}$ devient très inférieur à la distance d'extinction $\xi_{\mathrm{g}}$.

Mais la description du joint et son observation en microscopie électronique ne peuvent se baser uniquement sur le modèle géométrique et doivent tenir compte du champ de déformation élastique associé à des réseaux périodiques plans de dislocations. Une méthode relativement simple pour calculer ce champ élastique a été développée récemment par Rey et Saada [120-121], elle permet de dégager l'influence relative des termes de contraintes à grande distance et à courte distance et de préciser si une théorie géométrique est correcte ou incomplète. Cette approche, complémentaire à l'approche géométrique, devrait être utilisée dans l'analyse des réseaux de dislocations observés.

4.2.1 Contraste de diffraction. - L'observation en deux ondes ne peut être utilisée pour les dislocations primaires que si l'angle de désorientation entre grains est suffisamment important; elle est plus facile à réaliser pour les dislocations secondaires mais on est vite limité par les faibles valeurs de b. L'espacement minimum en dessous duquel les dislocations ne sont plus visibles a été calculé, dans le cas de la théorie à deux ondes, par Weatherly et Mok [122] :

$$
d_{\dot{\mathrm{D}}} \simeq 0,33 \xi_{\mathrm{g}} .
$$

Expérimentalement des distances inférieures à cette limite ont pu être appréciées (cf. § 4.2.2).

Le plus souvent, un nombre relativement important de faisceaux faibles sont excités; il n'est pas exclu que l'un d'entre eux puisse être utilisé pour obtenir une image de chaque dislocation, due à la déformation, notablement plus étroite. Cette technique du faisceau faible a en effet permis de résoudre des dislocations partielles peu espacées dans la matrice.

Mais, le plus souvent, les dislocations intrinsèques sont observées dans des conditions où les deux cristaux sont fortement excités. Si les faisceaux diffractés ne peuvent pas être séparés par le diaphragme de l'objectif, des franges de Moiré se superposent sur l'image aux réseaux de dislocations et il est important alors de ne pas confondre ces phénomènes périodiques d'origines différentes. Si l'on considère, par exemple, un joint de torsion de faible désorientation, la distance entre dislocations est donnée par :

$$
d_{\mathbf{D}}=\frac{|\mathbf{b}|}{\theta} .
$$

La distance des franges de Moiré dues à la faible rotation des plans $(k k l)$ des deux cristaux est :

$$
L=\frac{d_{h k l}}{\theta} \text {. }
$$

Un cas particulier peut donc exister lorsque $|\mathbf{b}|=d_{h k l}$ : les franges de Moiré sont confondues avec les lignes de dislocations. Un test facile permet de les distinguer :
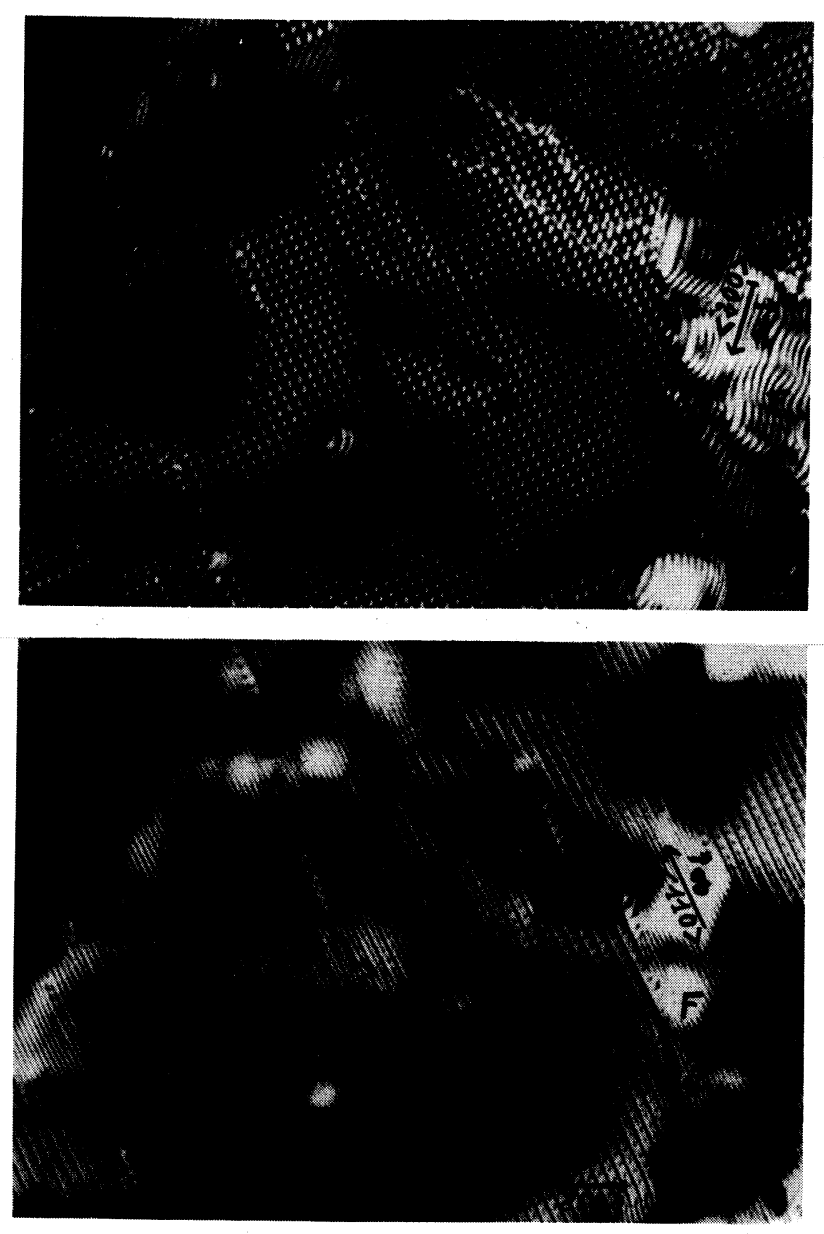

Fig. 39. - Différenciation des réseaux de dislocations intrinsèques et des franges de Moiré (celles-ci changent d'orientation avec $\mathbf{g}$ ); noter que dans le cas où $\mathbf{g}=\langle 110\rangle$, les dislocations vis perpendiculaires à ce vecteur ne sont pas visibles. Les franges de Moiré sont visibles seules en $F$ et $G$.

[Test to distinguish Moiré fringes from intrinsic dislocation arrays (single set of Moiré fringes in $F$ and $G$ always runs parallel to the average diffraction vector); In the case $g=\langle 110\rangle$, the screw dislocations perpendicular to $\mathbf{g}$ are not observed.] 
il suffit d'utiliser différents vecteurs de diffraction $\mathbf{g}$, les franges de Moiré parallèles à la direction moyenne de $\mathbf{g}$ changent d'orientation avec ce vecteur alors que le réseau de dislocations est fixe dans le plan du joint (Fig. 39). Il est évident que les franges de Moiré contribuent au contraste des dislocations même si elles sont inclinées par rapport au réseau. Cet effet de contraste conjoint a été étudié par Thölen [68] qui en réduisant progressivement l'espacement des dislocations obtient une image uniquement due à la désorientation des deux cristaux lorsque $d_{\mathbf{D}}<0,3 \xi_{\mathrm{g}}$. Ces résultats sont en accord avec ceux obtenus dans les conditions de deux ondes [122].

Mais, à nouveau, dans des conditions où les deux cristaux sont fortement excités, des réseaux de dislocations intrinsèques ont été résolus dans l'or lorsque $d_{\mathrm{D}} \simeq 18 \AA \simeq 0,08 \xi_{\mathrm{g}}[22,23]$. Le désaccord entre les prévisions théoriques et les résultats expérimentaux suggère, d'une part une appréciation erronée des contraintes et d'autre part, la possibilité d'intervention d'un autre mécanisme de contraste des dislocations.

4.2.2 Le contraste d'interférence. - a) Origine $d u$ contraste. - Le principe du phénomène est le suivant : tout faisceau d'électrons qui traverse le cristal supérieur peut être diffracté par le réseau bidimensionnel que constituent les contraintes périodiques dans le joint (Fig. 38). Ce réseau peut être compris comme une alternance, dans deux directions du joint, de rangées de forte et de faible densité atomique; il est analogue à une grille de diffraction pour laquelle il est possible de calculer un facteur de structure $[69,70]$. Le diagramme de diffraction produit par cette grille optique consiste en une série de taches de diffraction supplémentaires (extra-réflexions) disposées en carré autour de chaque réflexion du système C.F.C., par exemple, et ayant même orientation que le réseau de dislocations (Fig. 10). L'espacement entre dislocations étant $d_{\mathrm{D}}$, la distance entre la tache de diffraction de la matrice et chaque tache résultant de la diffraction du joint est donnée par :

$$
|p|=\frac{1}{d_{\mathrm{D}}} .
$$

Il est important de noter que lorsque $d_{\mathrm{D}}$ diminue, $|p|$ augmente, ce qui permet de distinguer plus aisément les taches dues au joint ; malheureusement, l'intensité de celles-ci diminuent avec l'épaisseur de la région du joint donc également avec $d_{\mathrm{D}}$

Dans le cas d'un joint faiblement désorienté, le cliché de diffraction présente les taches correspondant à un même plan $(h k l)$ dans les deux cristaux distantes de :

$$
q=\left|\mathbf{g}_{h k l}\right| \theta=\frac{\theta}{d_{h k l}}
$$

par ailleurs :

$$
|p|=\frac{1}{d_{\mathrm{D}}}=\frac{\theta}{|\mathbf{b}|}
$$

La relation entre le vecteur de Burgers des dislocations primaires (qui est le vecteur de Burgers de la matrice) et la distance des plans $(h k l)$ entraîne une relation entre $|p|$ et $|q|$. Par exemple, dans le cas $\mathrm{du}$ joint de torsion [001] dans l'or, pour la réflexion $(200)_{1,2}$ (Fig. 10)

$$
\begin{aligned}
& |p|=\frac{2 \theta}{a \sqrt{2}} \\
& |q|=\frac{2 \theta}{a} \\
& |p|=\frac{|q|}{\sqrt{2}}
\end{aligned}
$$

ce qui correspond biè à la configuration observée.

Cependant, il faut prendre soin de ne pas confondre les taches de diffraction provenant de la structure propre du joint avec des taches de double diffraction. L'analyse d'un diagramme de diffraction de bicristal est, en effet, très complexe car il contient, en principe, les réflexions dues :

- à la diffraction de chaque cristal ;

- à la double diffraction du cristal supérieur et inférieur ;

- à la double diffraction par l'un ou l'autre des cristaux et la région du joint ;

- à la diffraction résultant du champ périodique de déplacements dans le joint.

Donnons un exemple d'analyse d'un cliché aussi complexe dans le cas d'un pur joint de torsion [001], $\Sigma=13, \theta=22^{\circ} 62$ [22]. L'analyse est basée sur le fait que le réseau réciproque du plan (001) du C.S.L. (qui est le plan du joint) a une propriété particulièrement intéressante : ses points représentent toutes les réflexions possibles, soit réelles, soit doublement diffractées provenant du joint et des deux cristaux (Fig. 40). On sait, par ailleurs, que le réseau de dislocations primaires est une image du réseau «O $O$ ». Les réflexions dues uniquement au joint seront donc situées sur les sites du réseau réciproque du réseau « $\mathrm{O}$ » qui est une surstructure du réseau réciproque du C.S.L. Les réflexions propres au joint seront appelées réflexions $\mathrm{O}$; il convient de les distinguer parmi toutes les taches obtenues sur le cliché de diffraction. Sur la figure $40 b$, on voit qu'il est relativement aisé d'éliminer les taches de double diffraction dues aux deux cristaux; par contre, une tache telle que A peut provenir soit du joint seul, soit de la double diffraction par une réflexion du joint $B$ et une réflexion du cristal $C$; la seule manière de lever cette ambiguïté est d'effectuer une inclinaison du bicristal suffisamment importante pour que les éventuelles réflexions qui contribueraient à cette double diffraction soient écartées de la sphère d'Ewald (cette opération est plus aisée avec un porte-objet basculementrotation qui permet d'amener la tache à analyser sur l'axe de basculement); si la réflexion est conservée, elle provient réellement de l'interface : c'est une réflexion « $\mathrm{O} »$. 

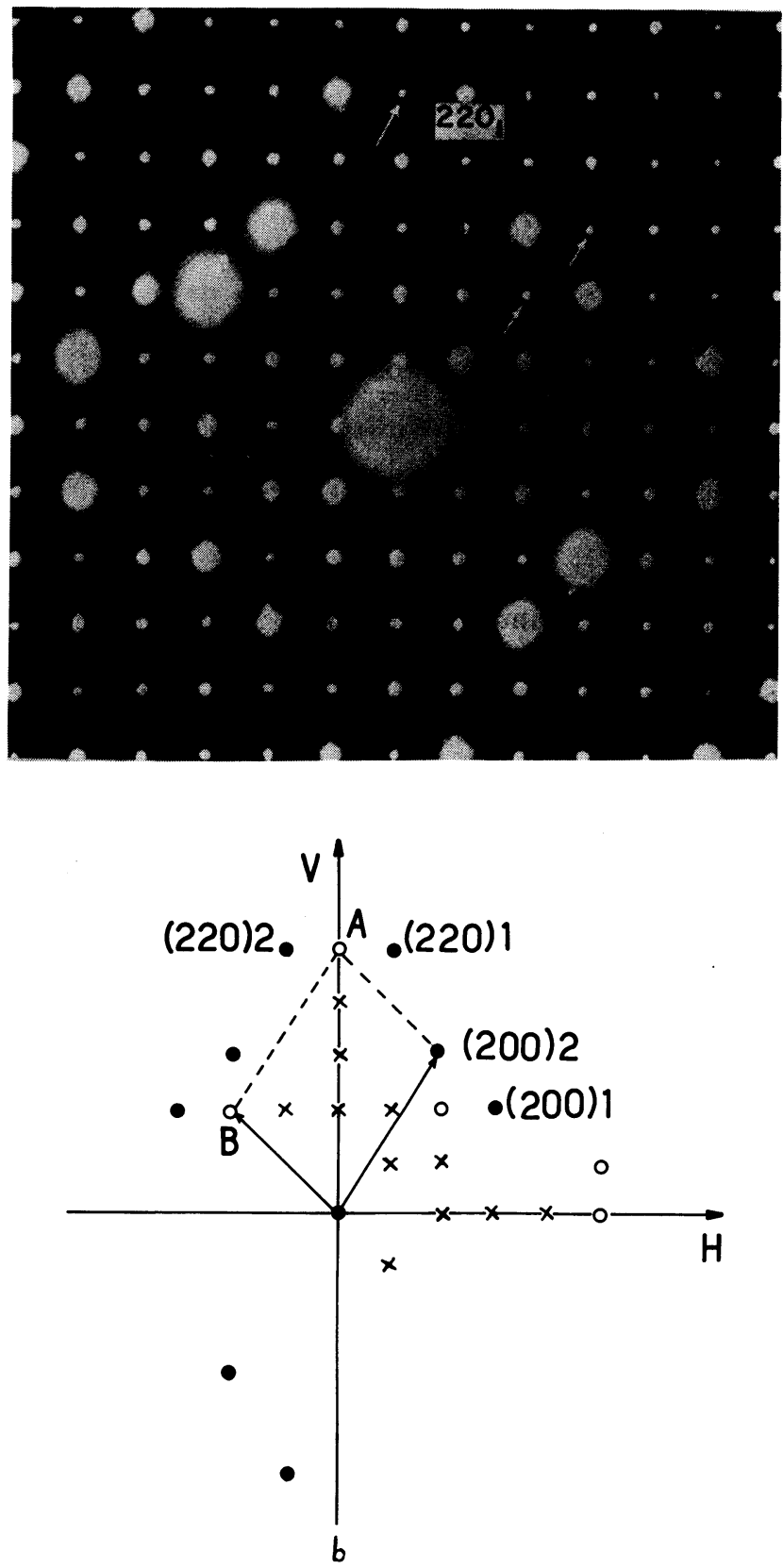

Fig. 40. - a) Diagramme de diffraction d'un joint de torsion [001] $\left(\theta=22^{\circ} 6\right)$. Les réflexions « $O$ » possibles sont indiquées par des flèches; $b$ ) Schéma d'une coupe [001] du réseau réciproque du réseau de coïncidence pour le joint précédent. Les vecteurs indiquent comment la réflexion « $\mathrm{O}$ » en $\mathrm{A}$ pourrait être produite par double diffraction entre une réflexion " $O$ » $(O)$ et une réflexion de la matrice $(0) ; \times$ : tache de double diffraction réelle (l'appartenance de $A$ aux réflexions « $O$ » est confirmée après basculement de l'échantillon autour d'une direction moyenne $\langle 110\rangle$ afin de diminuer la contribution éventuelle des taches B et $(200)_{2}$ en les écartant de la sphère d'Ewald [22].

[a) Electron Diffraction pattern from $\theta=22^{\circ} 6$ twist boundary; Possible « $\mathrm{O}$ » reflections are indicated by arrows ; $b$ ) Schematic 001 reciprocal lattice section of the C.S.L. for the previous boundary. The vector addition indicates how « $\mathrm{O}$ » reflection $\mathrm{A}$ can be produced by double diffraction between the F.C.C. reflection $(200)_{2}$ and the " $\mathrm{O}$ " reflection $\mathrm{B}$. Spots produced by double diffraction are indicated by the bicrystal was tilted about the average 110 direction so that the $B$ reflection and the $(200)_{2}$ B reflection were no longer on the Ewald sphere. The A reflection remained : it is a real « $\mathrm{O} »$ reflection.]
La contribution des réflexions d'ordre supérieur de la matrice aux réflexions " $O$ » a également été éliminée [123]. Cette précaution s'est avérée nécessaire compte tenu du rayon infini de la sphère d'Ewald en diffraction électronique. De ce point de vue, l'emploi de la diffraction des rayons $X$ pour l'étude de la structure des joints de grains apparaît comme une technique nettement préférable car les processus de diffraction multiple sont considérablement réduits [70].

L'attribution, sans ambiguïté, d'une extra-réflexion à la structure du joint permet, à partir de sa distance $|p|$ à la réflexion de $\mathrm{Bragg}$, de déterminer la périodicité du phénomène origine de cette réflexion c'est-à-dire l'organisation périodique, dans le joint, de dislocations distantes de $d_{\mathrm{D}}$. Rappelons qu'en utilisant le vecteur de Burgers de la matrice b et la relation qui relie b à $d_{\mathrm{D}}$ (cf. § 3.1.1), l'analyse des clichés de diffraction a permis de révéler l'existence des dislocations primaires pour des joints fortement désorientés jusqu'à $42^{\circ} 5$ (Fig. 4).

Cette analyse, déjà complexe dans le cas d'un joint bien défini cristallographiquement et parallèle à la surface de la lame, devient extrêmement difficile lorsqu'il s'agit d'un joint incliné dans un polycristal; pourtant elle est nécessaire et on se gardera de relier, sans une analyse fouillée du cliché de diffraction, l'apparition d'un phénomène périodique à la structure du joint propre [124].

b) Observation du contraste d'interférence. $-\mathrm{Si}$ les réflexions " $\mathrm{O}$ » voisines d'une tache de diffraction $(h k l)$ de la matrice ne sont pas éliminées par le diaphragme de l'objectif, elles peuvent interférer avec la réflexion de Bragg; l'image en fond noir correspondant à $\mathbf{g}_{k k l}$ présente alors des franges d'interférences dont la périodicité est celle du réseau " $O$ » et qui visualisent le réseau de dislocations primaires. $\mathrm{Ce}$ contraste d'interférence analogue à une image de réseau (lattice imaging) se superpose au contraste de contrainte; il devient même la principale source $\mathrm{du}$ contraste lorsque la distance entre dislocations diminue, d'autant plus que la réflexion " $\mathrm{O}$ " s'écartant de la réflexion de Bragg peut être optimisée $(\mathbf{s}=0)$ et explique pourquoi les dislocations sont visibles pour des distances inférieures à $0,33 \xi_{\mathrm{g}}$ (cf. § 4.2.1).

A titre d'exemple, dans un joint de torsion [001] dans l'or, les dislocations primaires sont visualisées essentiellement :

- par leur contraste de contrainte si $d_{\mathrm{D}}>34 \AA$;

- par leur contraste d'interférence si $d_{\mathrm{D}} \leqslant 18 \AA$.

Ainsi, le contraste d'interférence a permis d'observer des réseaux de dislocations primaires pour des angles de désorientation autour de [001] dans l'or allant jusqu'à $25^{\circ}(d \simeq 7 \AA)$ (cf. §3.1.1).

En ce qui concerne les dislocations secondaires, l'influence de leur structure périodique sur la diffraction de l'interface n'est pas clairement identifiée. Signalons cependant que, dans le cas d'un joint 
incliné du type Plane Matching, des effets dus à l'arrangement périodique des dislocations ont pu être détectés dans les clichés de diffraction sans ambiguité, mais, réciproquement, la visualisation de ces dislocations grâce au contraste d'interférence reste problématique [124].

4.3 Conclusion. - La microscopie électronique a permis un grand progrès dans la connaissance du joint de grains en donnant une réalité physique au modèle géométrique basé sur le formalisme de Bollmann. Elle apparaît actuellement limitée dans la mesure où elle ne permet pas de visualiser directement la structure de cœur du joint. Cependant le développement de méthodes tels l'observation en faisceau faible, l'image de réseau (microscopie à haute résolution), parallèlement, l'extension et les progrès faits dans l'emploi des simulations pour l'étude des contrastes conservent à la microscopie électronique en transmission une place de choix dans les moyens d'étude des interfaces.

Par ailleurs, elle reste la technique la plus opérante pour l'étude de la structure des joints réels «au hasard " des polycristaux, étude qui débouche sur la compréhension des propriétés d'emploi des matériaux.

Enfin, signalons que l'emploi de la haute tension permet l'observation des interfaces dans un échantillon métallique plus proche de l'état massif et la mise en œuvre d'expériences in situ fort utiles pour l'étude du comportement des joints de grains soumis à diverses sollicitations.

5. Approche analytique. Approche géométrique et propriétés du joint de grains. - 5.1 APPROCHE ANALYTIQUE. - Le principe de l'approche analytique est la recherche de la configuration des atomes dans le joint correspondant à l'énergie libre minimale grâce aux possibilités offertes par le calcul sur ordinateur (pour une bibliographie détaillée voir la référence [125]). Pour cela, on somme les énergies d'interactions entre atomes dans un volume fini autour du joint. Le système constitué par le joint et une certaine région des deux cristaux avoisinants est alors ajusté (ou relaxé) jusqu'à l'obtention d'un minimum énergétique. De nombreuses difficultés se présentent dans les différentes étapes du calcul :

- le choix du potentiel interatomique : généralement, les potentiels dits de force centrale faisant intervenir uniquement les distances entre atomes sont utilisés. Pour les joints de macle, certains modèles affinés tiennent compte des facteurs de structure électronique [126];

- la limitation de la zone de calcul ;

- la minimisation de l'énergie qui ne peut être que relative et conduire à une structure métastable ;

- les calculs sont faits, le plus souvent; avec des modèles statiques où les effets d'entropie sont négligés.

Les simulations sont limitées jusqu'à présent aux joints spéciaux de courte longueur d'onde

$\left(\lambda=|b| \Sigma^{1 / 2}\right)$.

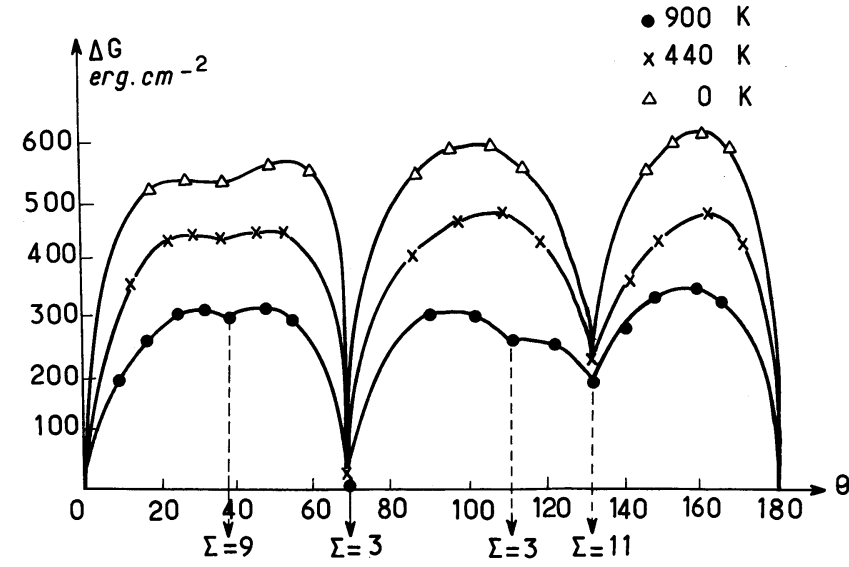

Fig. 41. - Influence de la désorientation sur l'énergie libre intergranulaire, à différentes températures, dans le cas des joints symétriques de flexion autour de [110], dans l'aluminium [5].

[Computed grain boundary energy at various temperature for symmetric [110] tilt boundaries in aluminium versus misorientation [5].]

Pour tester la validité d'un modèle, il convient si possible de comparer les résultats obtenus par le calcul à des résultats expérimentaux portant sur l'énergie [5], sur le déplacement relatif $\mathbf{R}$ d'un cristal par rapport à l'autre $[6,10]$, sur le facteur de structure déterminé par rayons $X$ [127].

En dépit des difficultés évoquées ci-dessus, des résultats fondamentaux ont été obtenus par les simulations à l'ordinateur :

- l'existence de minima énergétiques pour des angles de désorientation particuliers autour d'axes d'indices faibles (Fig. 41) [20] ;

- le cœur du joint est très étroit, de l'ordre d'une à deux distances atomiques ;

- la densité atomique reste relativement élevée dans le cœur du joint;

- enfin, très récemment, certains auteurs ont montré que la structure du cœur de tout joint pouvait être décrite par un arrangement de groupements polyédriques d'atomes (ou unités structurales de base), en nombre limité (Fig. 42) [7, 9, 128]. Ce modèle est similaire à celui des polyèdres compact d'atomes dans les liquides trouvé par Bernal [129]. Un joint spécial se distinguerait d'un joint général par l'existence d'un ordre à grande distance dans l'arrangement des polyèdres (Fig. 43) et on peut concevoir une continuité dans la description du joint dépendant du degré d'ordre présenté par cet arrangement.

Notons que cette conception récente du joint de grains semble donner un certain regain d'actualité à l'ancienne théorie de la couche amorphe mais en limitant considérablement l'épaisseur de cette couche et en profitant des progrès faits dans la connaissance de ces milieux. 

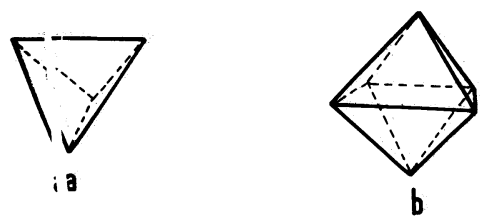

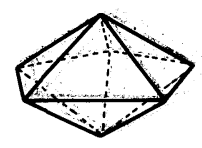

6

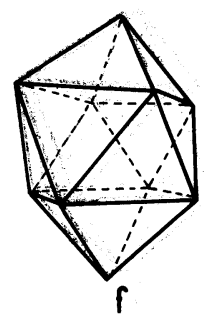

Fig. 42. - Les divers grc supements polyédriques d'atomes possible dans une interface [9].

[The different compact polyhedral groups of atoms in a grain boundary [9].]

(b)

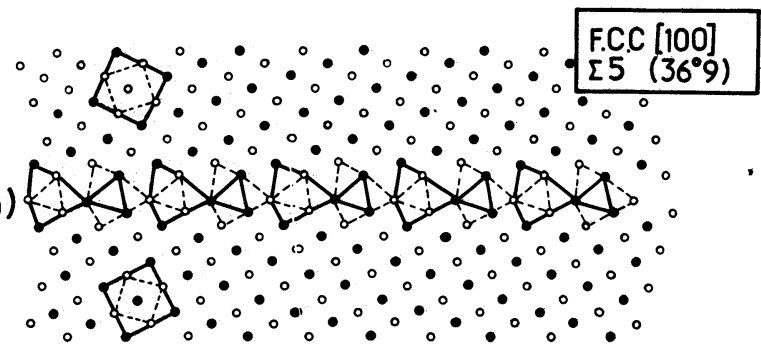

Fig. 43. - Arrangement régulier de polyèdres d'atomes dans un jớnt spécial; chaque unité correspond à une projection de la figure $42 e$

[Ordered array of compact polyhedra in a special grain boundary ; each unit is a projection of the figure $42 e$.]

\section{2 LIMITE COMMUNE ACTUELLE AUX DEUX MÉTHODES} D'APPROCHE. LES FACETTES DES JOINTS DE GRAINS. On peut remonter dans certains cas à la structure prévue par le réseau de coïncidence à partir de la configuration des atomes dans le plan du joint déterminée par les calculs énergétiques, c'est-à-dire que de rares recoupements peuvent exister entre les deux modèles [20]. Cependant les approches géométrique et analytique ne doivent pas être considérées en termes de comparaison mais en termes de complémentarité.

$\mathrm{Si}$ on considère leurs limites telles qu'elles se dégagent des études actuelles, une caractéristique fondamentale leur est commune : la structure et l'énergie d'un joint de grains ne dépendent pas seulement de la désorientation entre les grains voisins c'est-à-dire du nombre de coïncidence $\Sigma$. On a vu, par exemple (cf. § 3.1.2), qu'un joint de flexion [001], $\Sigma=5$, peut se décrire par des arrangements de dislocations secondaires différents du point de vue de leur orientation et de leur vecteur de Burgers selon que le plan du joint est un plan (010) ou un plan (110) (Fig. 19). Par ailleurs, la courbe de la figure 41 donnant l'énergie calculée d'un joint de flexion [110] dans l'aluminium en fonction de la désorientation $\theta$ présente un minimum très prononcé pour $\Sigma=3$ lorsque $\theta=70^{\circ} 5$ alors qu'il n'y a pas de chute énergétique pour la même coïncidence lorsque $\theta=109^{\circ} 5$ (cf. $\S 5.1$ ).

Ces constatations mettent en évidence le rôle fondamental joué par le plan du joint, non pris en considération dans la plupart des travaux. Or, un minimum énergétique est associé à une orientation particulière de l'interface qui peut entraîner sa décomposition en facettes [32, 130, 131]. L'existence d'un joint plan ou d'un joint en facettes s'explique pour des raisons purement thermodynamiques (Fig. 44) : si $A$ et $\gamma$ sont respectivement les aires et les énergies interfaciales des différentes faces, la condition d'équilibre d'un joint plan est :

$$
A_{3} \gamma_{3}<A_{1} \gamma_{1}+A_{2} \gamma_{2} \text {. }
$$

L'apparition des facettes et leurs orientations peuvent être déduites d'une construction de WulffHerring (Fig. 45) [132]. Mais, jusqu'à présent, les énergies utilisées dans ce type de construction étaient déduites d'une hypothèse de base du modèle géométrique à savoir que le plan du joint passe autant que possible par les sites du réseau de coïncidence; les minima d'énergies correspondaient aux plans ayant une grande densité de sites communs aux deux réseaux cristallins. Des travaux récents ont montré que d'autres facteurs interviennent dans la sélection des facettes, les minima d'énergie ne sont pas liés étroitement à des considérations géométriques [133]. Il y a là une insuffisance fondamentale dans l'approche basée sur le formalisme de Bollmann.

Par contre, bien qu'il n'existe aucune étude énergétique sur les facettes effectivement rencontrées dans différents métaux, le calcul des énergies de différents plans pour une même désorientation est théoriquement possible. Potentiellement, la méthode analytique présente, de ce point de vue, un avantage essentiel. En effet, on peut toujours envisager une construction du type Wulff-Herring dans laquelle on utiliserait les minima d'énergie calculés. Actuellement,

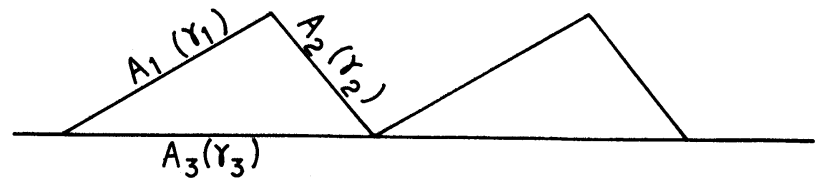

Fig. 44. - Schéma montrant la décomposition d'un joint en facettes.

[Faceting of grain boundary.] 


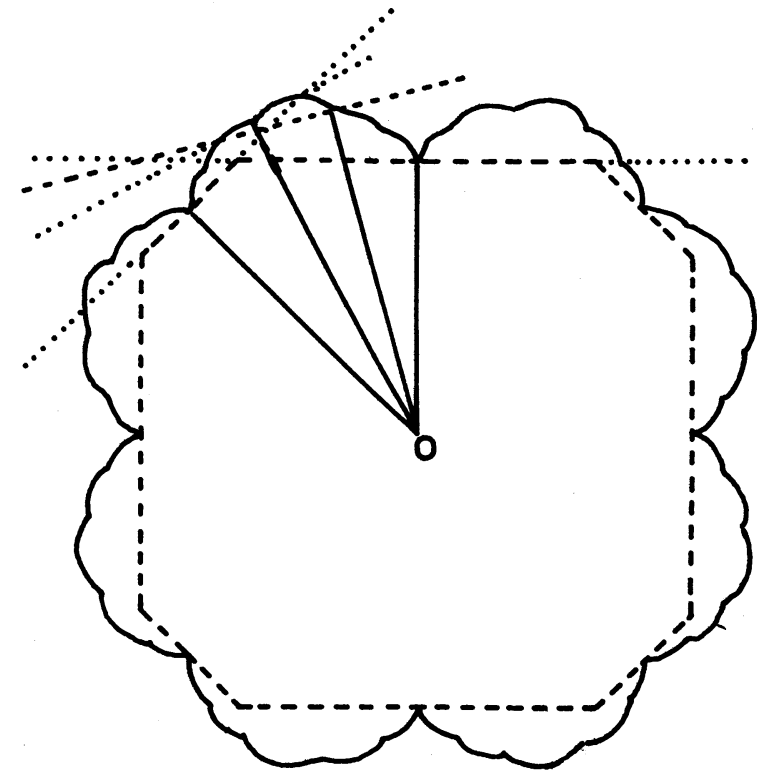

Fig. 45. - Construction de Wulff-Herring [132] :- - diagramme polaire des énergies de surface $;. \ldots$ : plans normaux aux rayons de la courbe énergie ; .... : profil d'équilibre.

[Wulff-Herring plot [132] : - : Polar plot of surface energy .... : Samples of planes normal to radius vectors of this plot; -... : Equilibrium profile.]

les deux modèles ont en commun de ne pas prendre en compte le plan du joint. Mais, pour le modèle géométrique, il s'agit d'une inadéquation de principe alors que, pour le modèle analytique des développements ultérieurs peuvent pallier cette insuffisance.

Cette restriction commune devra être présente à l'esprit dans la suite de cet article concernant l'étude des propriétés des joints de grains.

5.3 INTÉRÊT RESPECTIF DES MODÈLES ANALYTIQUE ET GÉOMÉTRIQUE POUR L'ÉTUDE DES PROPRIÉTÉS DU JOINT DE GRAINS. - 5.3.1 Généralités sur les propriétés des interfaces. - $\mathrm{Si}$ on compare les régions de bon matériau (grains) et les régions de mauvais matériau (joints de grains), on peut établir un parallélisme entre :

- la structure intrinsèque du joint et le cristal parfait ;

- la structure extrinsèque du joint et le cristal hors d'équilibre.

Poursuivant cette comparaison, on peut concevoir alors que certaines propriétés du joint s'interprètent en considérant principalement sa structure d'équilibre (périodicité-anisotropie) et que d'autres sont liées plus particulièrement aux écarts à l'équilibre. Il est évident que dans le premier cas, le modèle analytique est d'un apport précieux dans la limite où il s'agit de joints spéciaux alors qu'il est inopérant actuellement pour l'étude des propriétés liées non seulement au désordre du joint spécial mais à l'équilibre dans les joints généraux.

Cette distinction entre deux types de propriétés est cependant formelle, comme elle l'est d'ailleurs dans le cristal, pour diverses raisons :

- la structure extrinsèque dépend de la structure intrinsèque,

- une propriété peut s'interpréter sur la base de l'ordre et être profondément modifiée par toute perturbation de cet ordre (dans un cristal, la diffraction des rayons $\mathrm{X}$ ou des électrons qui s'explique par l'arrangement périodique des atomes est fortement influencée par toute rupture de périodicité, ce qui en constitue l'intérêt);

- l'interdépendance des propriétés (on sait par exemple que les ségrégations modifient la diffusion et la corrosion intergranulaires et la mobilité des interfaces).

Malgré les limites de cette classification, compte tenu des possibilités différentes d'utiliser les modèles géométrique et analytique, nous tenterons de dégager successivement l'intérêt respectif de ces modèles pour l'étude des propriétés en relation avec :

- la structure intrinsèque des joints de grains

- la structure extrinsèque des joints de grains sans perdre de vue la complexité et l'imbrication étroite des phénomènes $\left({ }^{7}\right)$.

5.3.2 Propriétés du joint et structure intrinsèque. Nous avons regroupé dans cette catégorie des propriétés présentant les caractéristiques suivantes :

- elles peuvent s'interpréter sans qu'il soit nécessaire de prendre en compte les dislocations extrinsèques :

- plus généralement, on peut les concevoir en isolant fictivement l'interface, c'est-à-dire qu'on peut négliger l'intervention du milieu extérieur constitué par les grains voisins ;

- en liaison avec la caractéristique précédente, elles ne concernent qu'une région très étroite de part et d'autre du plan du joint (une ou deux distances atomiques),

- des relations quantitatives ont pu être établies entre des paramètres qui décrivent ces propriétés et la désorientation entre deux grains autour d'un même axe.

a) Diffraction. - La découverte que le joint de grains peut constituer une grille de diffraction pour les électrons et les rayons X est évidemment imputable uniquement au modèle géométrique. Rappelons que ce phénomène a permis de révéler l'existence de dislocations primaires dans les joints de grains à grand angle affirmant ainsi la validité du réseau « $\mathrm{O}$ » (cf. \$ 4.2.2).

b) Diffusion intergranulaire. - La diffusion aux joints de grains est nettement plus rapide que la diffusion en volume surtout aux basses températures ; on la caractérise, dans un polycristal, par un coefficient

$\left.{ }^{7}\right)$ Cette proposition de classification ne constitue en sorte qu'un canevas pour les discussions. 


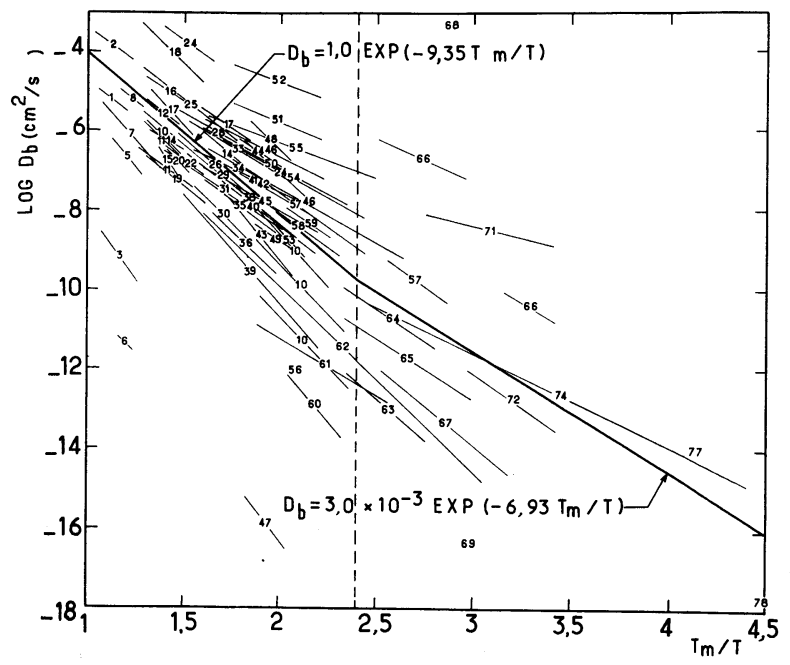

Fig. 46. - Diagramme d'Arrhenius donnant tous les coefficients de diffusion intergranulaires disponibles actuellement dans les métaux polycristallins. Les nombres indiquent les références de l'article [134].

[Arrhenius plot of all presently available grain boundary diffusivities in polycristalline metals. Numbers indicate the references list in [134].]

de diffusion intergranulaire $D_{\mathrm{j}}$ qui est un coefficient statistique. Les mesures de ce coefficient sont en général imprécises comme le montre la grande dispersion des données actuelles sur les métaux (Fig. 46) [134]. En effet, la diffusion aux joints dépend :

- du type de joint (périodicité);

- de la direction de diffusion dans le plan du joint (anisotropie),

elle est donc fonction de la structure intrinsèque $d u$ joint de grains. Mais s'agit-il de cette structure telle qu'elle est décrite par l'arrangement des dislocations primaires et secondaires ou de la structure de cœur telle qu'elle est décrite par l'arrangement de polyèdres d'atomes ? La plupart des résultats expérimentaux prouvent que c'est le modèle énergétique lié à la structure de cœur du joint qui est le mieux corrélé au phénomène diffusionnel. Pour certaines désorientations autour d'axes simples, les joints présentent simultanément un minimum énergétique et un minimum de diffusion (Fig. 47) [135]. Cependant, le lien énergie-diffusion est loin d'être biunivoque [136, 137].

Le modèle géométrique, quant à lui, n’a été utilisé avec succès que dans le cas de l'autodiffusion intergranulaire dans un bicristal d'argent parallèlement à l'axe de flexion $\langle 100\rangle$ [138]. A l'alignement des dislocations primaires suivant la direction $\langle 100\rangle$ correspond une série de canaux le long desquels peut se faire la diffusion d'autant mieux que le nombre de ces canaux est plus grand (Fig. 48); la diffusivité doit alors être inversement proportionnelle à la distance des dislocations primaires; c'est ce qui a été constaté, avec une bonne approximation, pour des désorientations allant jusqu'à $16^{\circ}$, la distance des dislocations primaires est alors sensiblement égale à 4 paramètres cristallins ; cette limite de validité du modèle suggère que la région de diffusion rapide autour du joint ne s'étend pas au-delà d'un à deux paramètres cristallins en accord avec la largeur du joint habituellement considérée. La même analyse n'a cependant pu être étendue à d'autres métaux. Par ailleurs le modèle géométrique ne permet pas d'expliquer la contradiction qui existe dans les valeurs respectives des pénétrations du zinc dans l'aluminium parallèlement et perpendiculairement à l'axe de rotation $\langle 011\rangle$ : ce sont les joints spéciaux où l'anisotropie est la plus forte pour lesquels l'écart est minimal (Fig. 47).

Si les paramètres géométriques du joint ont donc une nette influence sur la diffusion intergranulaire, il est peu probable que l'on puisse la comprendre par l'approche géométrique; les considérations éner-

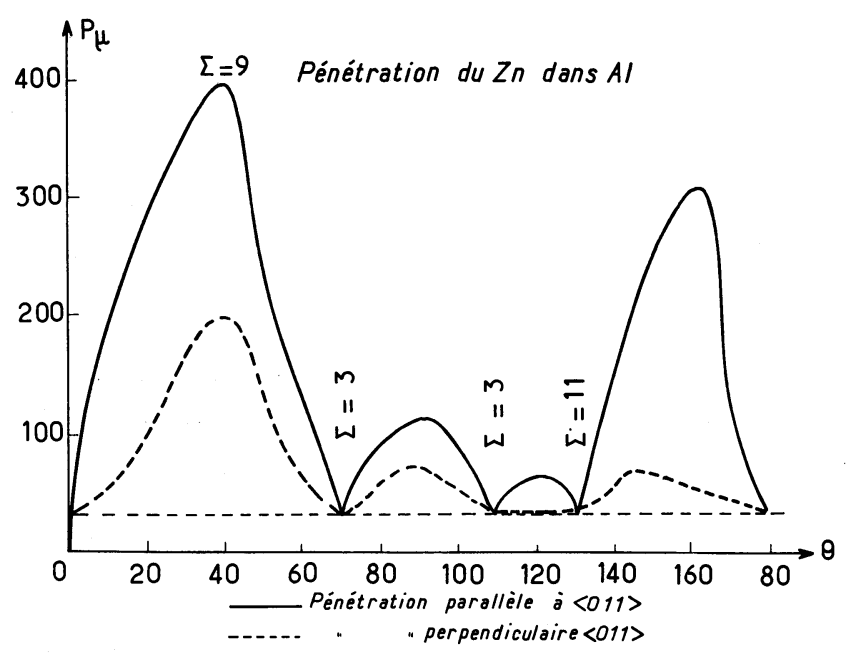

Fig. 47. - Pénétration du zinc dans des bicristaux d'aluminium, de flexion symétrique autour de [011] [135].

[Penetration of zinc into [110] tilt boundaries of aluminium, parallel and perpendicular to the tilt axis [135].]

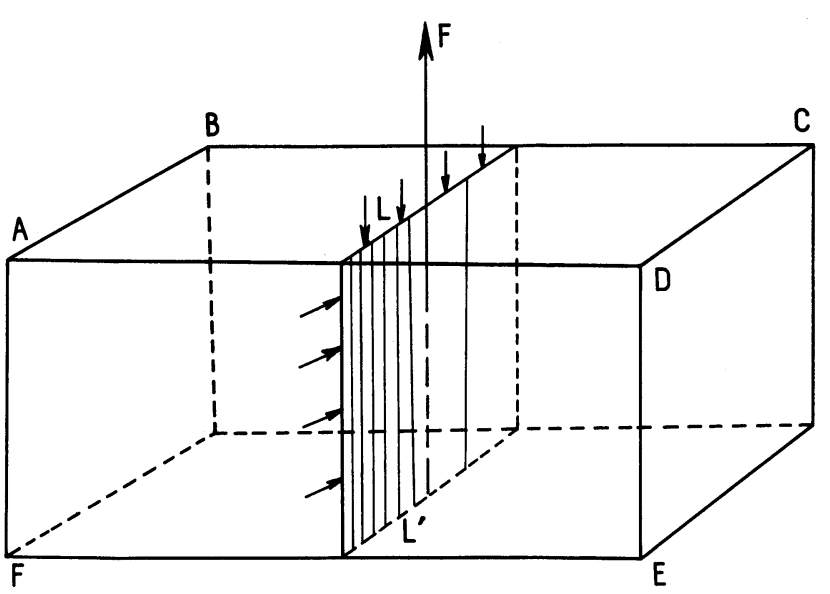

Fig. 48. - Schéma montrant l'existence de canaux parallèles à l'axe de flexion dans un joint faiblement désorienté; ces canaux sont des chemins faciles de diffusion ou de corrosion.

[Schematic illustration of rapid diffusion and corrosion pipes along the tilt axis in a low angle tilt boundary.] 
gétiques semblent également limitées. Il faut pouvoir remonter au mécanisme atomique de la diffusion intergranulaire qui est difficile à établir [139]. On peut dire cependant que l'approche analytique est prometteuse dans la mesure où elle ne se limite pas à la détermination de l'énergie globale du joint mais qu'elle peut donner une répartition de celle-ci entre les différents sites et atteindre ainsi une idée de la densité de lacunes dans le joint [20]. En effet, si la diffraction s'explique intégralement dans le grain sur la base du cristal parfait et repose intégralement dans le joint de grains sur la structure intrinsèque, de même, la diffusion en volume qui, dans le cristal, fait intervenir la présence de lacunes nécessite, dans l'interface, l'existence de défauts identiques; or si les joints de grains, notamment généraux, ont été reconnus comme sources et puits de défauts ponctuels [140], peu de descriptions, à notre connaissance, ne tiennent réellement compte de ces défauts [141]. De ce point de vue, des progrès peuvent être espérés sur la base du récent modèle de l'arrangement polyédrique $[8,9,128]$.

c) Corrosion. - Les joints de grains sont, en général, plus sensibles à l'attaque par un milieu agressif que les cristaux pour des raisons thermodynamiques et cinétiques. L'intensité du courant de dissolution anodique s'exprime par une relation de la forme :

$$
I=I_{0} \exp (b V)
$$

où $I_{0}$ et $b$ sont, en général, considérés comme constants en cinétique électrochimique. Dans ce cas, on peut concevoir que les différences d'énergie entre les joints de grains et la matrice peuvent entraîner des différences de potentiel $\Delta V$ entre ces deux régions :

$$
V=-\frac{\Delta G}{Z F}
$$

et, de ce fait, une modification des courants de dissolution respectifs [142]. Mais, lorsqu'on applique un potentiel $V$ constant à un échantillon (méthodes potentiostatique et potentiocinétique), les courants de dissolution anodique correspondant à deux régions de cet échantillon pourront différer seulement si les termes pré-exponentiels $I_{0}$ diffèrent

$$
I_{0}=K \exp -\frac{\Delta G}{R T}
$$

$I_{0}$ est plus élevé dans une interface que dans un grain, plus élevé dans un joint général que dans un joint spécial pour deux raisons étroitement liées :

- une augmentation du nombre de sites actifs dans les régions désordonnées;

- une diminution de l'énergie d'activation de la corrosion correspondant à l'apport d'énergie $\Delta G$ d'une région par rapport à l'autre [142].

Ces considérations permettent donc de prévoir qu'un joint spécial sera plus résistant à la corrosion qu'un joint général quelle que soit l'approche de la connaissance du joint utilisée, énergétique ou géométrique.

En utilisant plusieurs critères de corrosion (profondeur d'attaque $H$, angle d'ouverture $\alpha$ et largeur du sillon intergranulaire $L$ ), pour divers métaux et divers types de joints, dans des milieux d'attaque différents, plusieurs auteurs ont mis en évidence l'existence de minima de corrosion corrélés à des minima énergétiques (les références à la plupart de ces travaux peuvent être trouvées dans l'article de Froment [143]). Un exemple est donné sur la figure 49 où l'on peut constater, en comparant avec la courbe énergétique (Fig. 41) que certaines contradictions apparaissent, comme dans le cas de la diffusion. Par ailleurs, il a été mis en évidence une anisotropie de profondeur d'attaque selon qu'elle a lieu parallèlement ou perpendiculairement à un axe de flexion. Mais l'interprétation de ce phénomène sur la base du schéma précédent (Fig. 48) a nécessité la prise en compte du processus de corrosion, en particulier de l'élimination des produits qui en résultent [144].

Cette intervention des mécanismes électrochimiques, souvent mal définis, rend extrêmement complexes l'établissement d'un lien entre corrosion et structure ou corrosion et énergie. Néanmoins, récemment, Beaunier a établi un modèle de description cristallographique de la structure d'un joint en considérant une association de zones de cohésion $C$ et de décohésion $D$ dont l'importance relative $D / L$ a permis d'expliquer avec succès la corrosion intergranulaire en fonction de l'angle de désorientation $\theta$ et d'un paramètre d'asymétrie $\phi$ [145-146]. Ce modèle a l'intérêt de prendre en compte indirectement le plan du joint lié au paramètre d'asymétrie $\phi$ mais, d'une part, il est limité aux joints spéciaux, d'autre part, il s'appuie sur l'hypothèse que le plan du joint

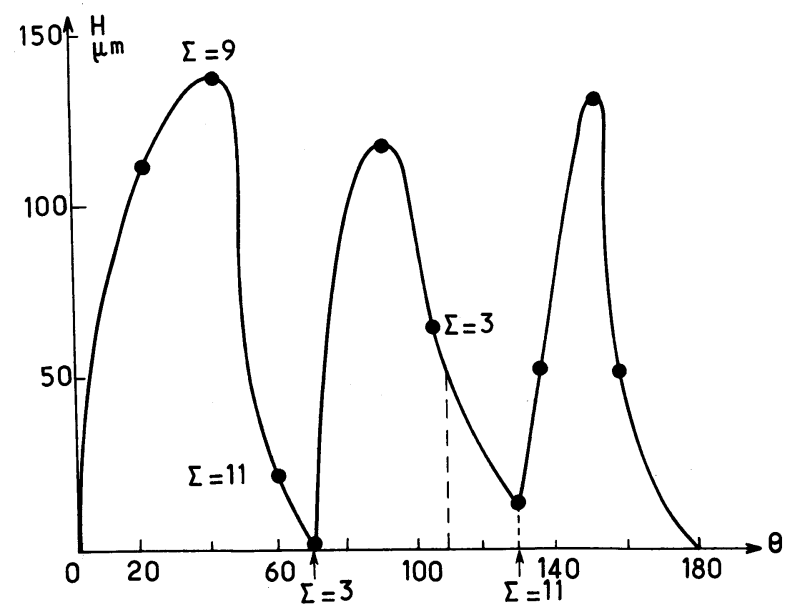

Fig. 49. - Influence de la désorientation $\theta$ sur la profondeur d'attaque $H$ de bicristaux d'axe de flexion [110] après maintien dans l'eau pressurisée à $150^{\circ} \mathrm{C}$ [144].

[Depth of boundary groove in aluminium [110] symmetrical tilt bicrystals corroded for 48 hours in water at $150^{\circ} \mathrm{C}$ as a function of the misorientation $\theta$ [144].] 
est un plan cristallographique simple du réseau de coïncidence, ce qui ne correspond pas toujours à la réalité (cf. $\$ 5.2$ ).

Comme dans le cas de la diffusion, c'est l'analyse de la position des atomes dans l'interface qui peut conduire à différencier des arrangements plus ou moins sensibles à l'agressivité d'un milieu par analogie à l'anisotropie bien connue de la corrosion des différentes faces d'un cristal.

d) Fluage intergranulaire. - Bien que des modèles basés sur le déplacement dans le plan du joint soit des dislocations intrinsèques, soit des dislocations extrinsèques aient été suggérés pour expliquer le fluage intergranulaire [147-148] nous avons fait choix de classer cette propriété comme dépendante plus étroitement de la structure intrinsèque pour les raisons suivantes :

- les dislocations extrinsèques disparaissent rapidement à température relativement élevée, la vitesse du phénomène étant considérablement supérieure à celle du fluage [52-54]. Ce phénomène est analogue à une recristallisation du joint [90]. Le fluage se propage donc dans une région sans défauts extrinsèques;

- les joints spéciaux sont moins sensibles au fluage intergranulaire que les joints généraux [149], ce qui semble en contradiction avec un rôle éventuel des dislocations extrinsèques; en effet, celles-ci sont plus stables dans les joints proches de la coïncidence que dans les joints généraux où elles se dispersent [5254]. Cette constatation peut signifier soit que le fluage ne nécessite pas la présence de dislocations extrinsèques, soit qu'il peut intervenir lorsque les vecteurs de Burgers de ces dislocations sont infiniment petits mais elles n'ont alors aucune signification physique ;

- des relations quantitatives ont été établies entre la vitesse ou l'énergie de fluage intergranulaire et la désorientation $\theta$ pour un même type de joint (Figs. 50 et 51) $[150-151]$;

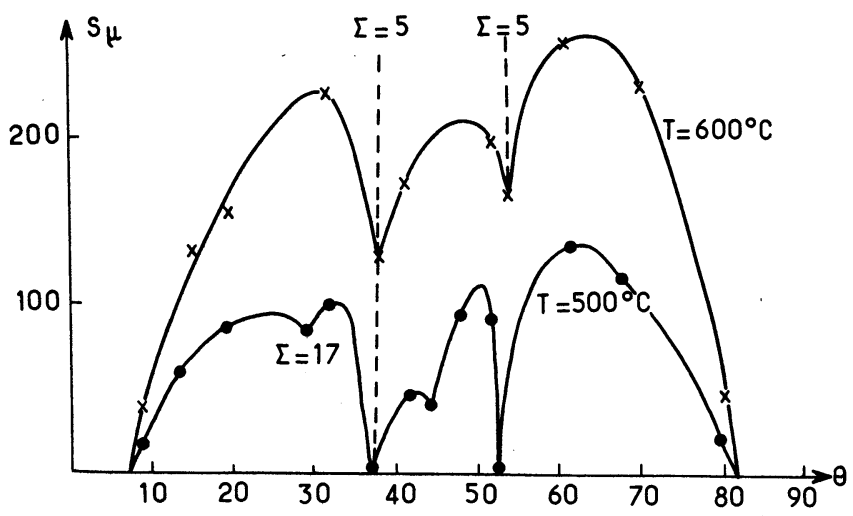

Fig. 50. - Influence de la désorientation sur le fluage d'un joint de flexion 100 dans l'aluminium sollicité

- à $500^{\circ} \mathrm{C}$ par une cission de $40 \mathrm{gf} / \mathrm{mm}^{2}$ pendant $100 \mathrm{~min}$.

- à $600{ }^{\circ} \mathrm{C}$ par une cission de $20 \mathrm{gf} / \mathrm{mm}^{2}$ pendant $100 \mathrm{~min}$. [150].

[Grain boundary sliding at $500^{\circ} \mathrm{C}$ under a shear stress of $40 \mathrm{gm} . \mathrm{mm}^{-2}$ and at $600^{\circ} \mathrm{C}$ under a shear stress of $20 \mathrm{gm} . \mathrm{mm}^{-2}$ after $100 \mathrm{~min}$., in aluminium symmetrical [100] tilt bicrystals [150].]

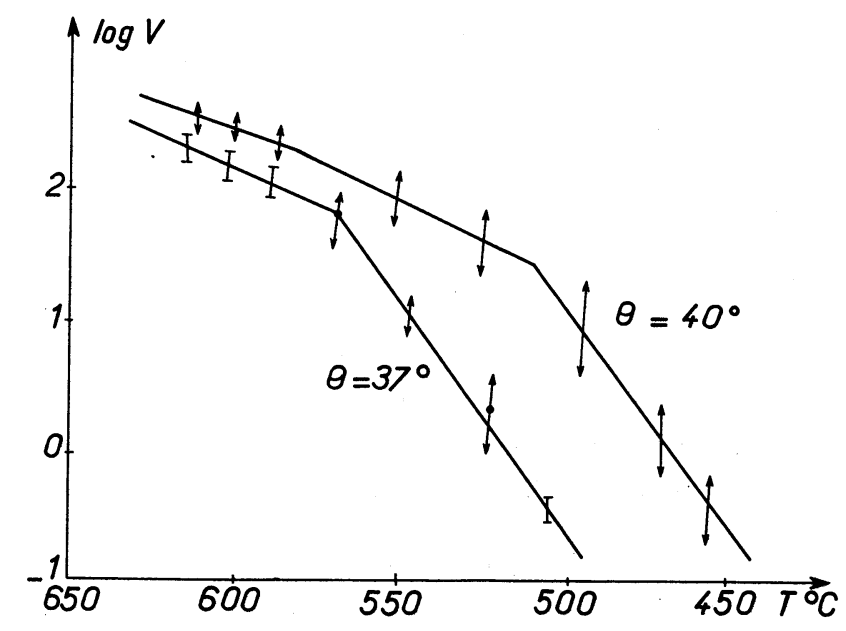

Fig. 51. - Influence de la température sur la vitesse initiale de fluage de bicristaux d'aluminium, désorientés de $40^{\circ}$ et $37^{\circ}$ autour d'un axe de flexion 100 [150].

[Effect of temperature on sliding rate of $37^{\circ} \mathrm{CSL}$ and $40^{\circ}$ random aluminium [100] symmetrical tilt boundaries [150].]

- si on fait une analogie avec la déformation à chaud d'un cristal, on peut concevoir pour une température inférieure à celle nécessaire dans la matrice, un comportement visco-plastique des joints. La déformation des verres peut être comparée au fluage intergranulaire, notamment des joints généraux, ce qui appuie la validité de la description polyédrique $\mathrm{du}$.joint de grains. Le fluage peut se concevoir comme un mouvement coopératif des arrangements d'atomes dans l'interface.

Le choix de cette classification du fluage intergranulaire est, bien entendu, criticable de plusieurs points de vue, entre autres :

- des mécanismes sont proposés dans lesquels les dislocations de matrice seraient retenues à la surface de part et d'autre du joint [152];

- les joints spéciaux fluent peu or ils possèdent, plus que tout autre, une structure intrinsèque mais, les vecteurs de Burgers étant importants, on peut concevoir une difficulté au mouvement des dislocations d'équilibre.

La limite essentielle pour la compréhension du fluage intergranulaire réside dans le fait qu'il intervient dans les joints généraux qui, actuellement, ne sont pas décrits ni par le modèle géométrique, ni par le modèle analytique.

e) Conclusion. - Pour toutes les propriétés regroupées dans ce chapitre, c'est le modèle d'arrangement de polyèdres atomiques dans l'interface qui laisse espérer un progrès dans leurs interprétations. Ce modèle permet, entre autres, d'envisager l'existence de défauts interfaciaux analogues aux défauts ponctuels dans la matrice alors que le modèle géométrique ne distingue qu'entre une structure parfaite du joint, comparable au cristal parfait, et une structure hors d'équilibre du joint, comparable au cristal écroui. 
Enfin, bien que ces propriétés peuvent se comprendre théoriquement en considérant uniquement la région du joint de grains, expérimentalement une restriction à leur étude réside dans l'impossibilité d'isoler le joint de son environnement. En fait, l'intervention de la matrice (échange de matière joint - volume pour la diffusion, effet de pile pour la corrosion) est importante même si on s'affranchit de la présence d'impuretés c'est-à-dire de la possibilité de ségrégation intergranulaire, en utilisant des matériaux de haute pureté. On peut donc se demander quelle est la réalité physique des propriétés intrinsèques : leur considération doit être vue comme une étape formelle dans la connaissance des joints; en dernière analyse, le milieu voisin de l'interface n'est pas seulement influant sur ces propriétés, il est déterminant.

5.3.3 Propriétés du joint et structure extrinsèque. - Ces propriétés ont en commun les points suivants :

- elles nécessitent l'intervention des dislocations extrinsèques,

- leurs interprétations tiennent obligatoirement compte des échanges avec la matrice avoisinante,

- des différences existent entre les joints spéciaux et les joints généraux mais il n'y a pas de relations bien établies en fonction de la désorientation,

- peu d'études énergétiques de ces propriétés ont été entreprises.

a) Déformation plastique. - L'analogie avec le cristal laisse entrevoir le rôle spécifique des dislocations extrinsèques qui sont analogues aux dislocations de matrice. La déformation d'un polycristal nécessite que les dislocations de chaque -cristal puissent traverser les interfaces. Dans le cas idéall où les vecteurs de Burgers des grains de part et d'autre du joint sont parallèles (ou quasi parallèles), le joint ne constitue aucune barrière. Mais, le plus souvent, le passage s'effectue en laissant une cont rainte associée à une ou plusieurs dislocations extrinsèques dans l'interface [153]

$$
\begin{aligned}
\mathbf{b}_{1} & =\mathbf{b}_{2}+\mathbf{b}_{\text {ext. }} \\
\mathbf{b}_{\text {ext. }} & =\sum \mathbf{b}_{\text {D.s.c. }} .
\end{aligned}
$$

Les réactions de dissociation de la dislocation de matrice dans le joint sont donc fondamentales; en particulier, les longueurs des vecteurs de Burgers des dislocations formées expliquent leur réalité physique et leur stabilité, ce qui peut constituer des critères de distinction entre joints spéciaux et joints généraux (cf. § 3.4.2).

Bien entendu, la vitesse des réactions de dissociation dépend du caractère des dislocations formées et de la température ; si les dislocations sont de caractère vis, elles peuvent glisser à température ambiante, si elles sont de caractère coin, leur déplacement se fait le plus souvent par montée activée thermiquement.

Les joints spéciaux qui peuvent retenir les dislo- cations extrinsèques s'opposent au passage ulitérieur des dislocations de matrice et constituent en quelque sorte, des joints durs, alors que les joints gén éraux dans lesquels il y a répartition de la contrainte constituent des puits efficaces pour les dislocations des cristaux : ce sont des joints mous [99].

Le modèle géométrique qui permet la détermination des paramètres cristallographiques des dislocations extrinsèques est spécialement bien adapté à l'étude de la déformation plastique des joints alors que le modèle énergétique est difficilement exploitable, compte tenu de la complexité de détermination des champs de contrainte (cf. §4.1).

b) Migration des interfaces. - Lors de la recristallisation, on sait que les joints se déplacent des régions à faible densité de dislocations vers les régions à forte densité de dislocations, en laissant les régions balayées libres de contrainte. Cette constatation suggère une interaction du joint en mouvement avec les dislocations de matrice avec pour résultat la création de dislocations extrinsèques.

Lors de la migration induite sous contrainte à haute température, il a été montré que les joints spéciaux peuvent migrer aisément alors que les joints généraux ne migrent pas et sont soumis à un fluage [149]. Or, seuls les joints spéciaux peuvent retenir les dislocations extrinsèques car la vitesse de dispersion est lente dans ces joints même à température élevée [52-54].

Il semble donc, dans les deux cas précédents, que la migration d'une interface fait intervenir les dislocations extrinsèques. Ceci ne signifie pas, pour autant, que le mécanisme de base de la migration repose uniquement sur la structure hors d'équilibre du joint car il implique aussi nécessairement un phénomène diffusionnel $[154,155]$.

Le transport de matière peut se concevoir par la propagation d'une marche associée à une dislocation extrinsèque $[61,50,156]$.

Dans le cas de la recristallisation, le nombre de dislocations de matrice qui s'incorporent dans le joint est élevé, l'activation thermique favorise leur déplacement conduisant soit à une annihilation [157], soit à un arrangement périodique duquel résulte un changement de structure du joint [53]; dans ce dernier cas, notons que les dislocations de matrice incorporée sont devenues des dislocations intrinsèques du joint de grains [55].

Lorsque l'interface se déplace dans une matrice recristallisée, la structure du joint ne change pas au cours de sa migration. La translation d'un réseau à un autre impliquant le mouvement de dislocations extrinsèques peut se concevoir dans un joint spécial mais est inopérante dans un joint général où ces dislocations n'ont pas de réalité physique. Or, contrairement aux résultats antérieurs [158], la moins grande mobilité des joints éloignés de la coïncidence est actuellement controversée [133,159].

La différence entre joints spéciaux et joints généraux 
doit être recherchée dans leurs capacités différentes d'interaction avec les impuretés qui ségrégeraient plus facilement dans les joints généraux, en accord ave'c la plupart des résultats (Fig. 52) [64, 160, 161]. Notons qu'on peut interpréter l'influence des impuretés sur la mobilité des interfaces par une éventuelle stabilisation des dislocations extrinsèques par les atomes ségrégés, par analogie avec les atmosphères de Cottrell dans le cristal.

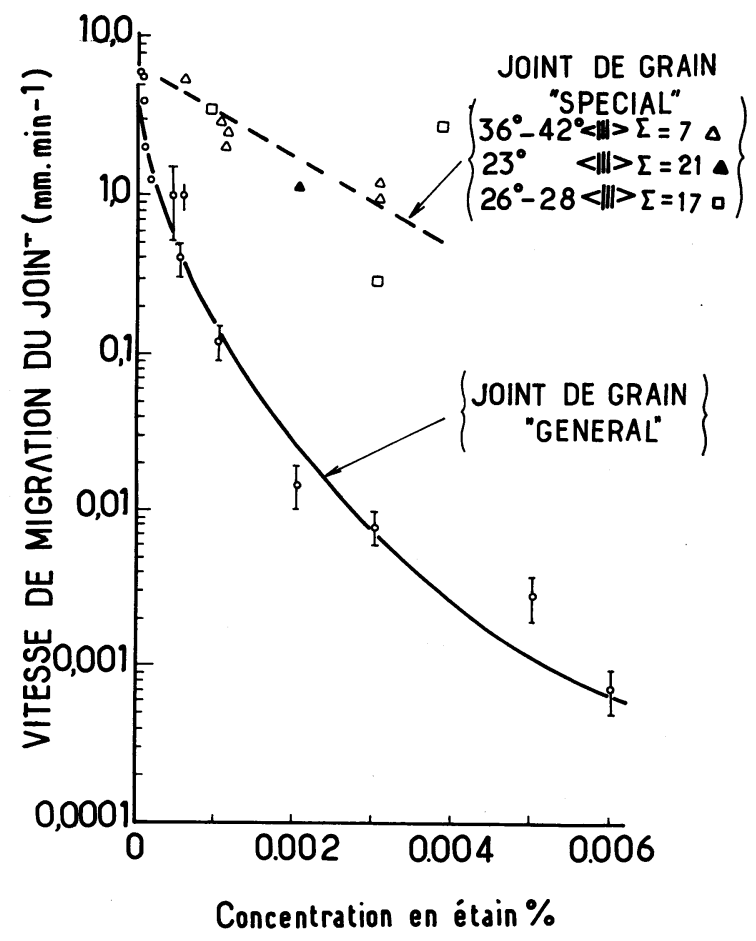

Fig. 52. - Influence de la teneur en étain sur les vitesses de migration à $300^{\circ} \mathrm{C}$ d'un joint de grain spécial et d'un joint de grain général dans un alliage plomb-étain [64].

[A comparison of the rate of grain boundary migration in lead-tin alloys at $300{ }^{\circ} \mathrm{C}$ versus tin content for general grain boundaries and several special grain boundaries [64].]

En conclusion, si le fait que la migration d'une interface dépend de sa structure atomique et même électronique [162] reste problématique, il nous semble difficile d'imaginer un processus dans lequel les dislocations extrinsèques (liées ou non à des impuretés) ne seraient pas prises en compte, en association avec le mécanisme diffusionnel. C'est pourquoi l'approche géométrique conserve un grand intérêt pour l'étude de la migration, en dépit des difficultés que les ségrégations, quasi inévitables, apportent à la compréhension du rôle de la structure seule.

c) Ségrégation. - Nous considérerons essentiellement la ségrégation d'équilibre d'une impureté c'est-à-dire que sa teneur globale est inférieure à sa limite de solubilité dans le métal.

Théoriquement, la ségrégation s'explique par la diminution de l'énergie superficielle résultant de l'adsorption d'atomes à la surface du joint selon l'expression classique de Gibbs, valable pour un système binaire dilué :

$$
\Gamma=-\frac{1}{R T} \cdot \frac{\mathrm{d} \gamma_{\mathrm{B}}}{\mathrm{d} \log X}
$$

où $\Gamma$ représente l'excès au joint (en moles $\left./ \mathrm{cm}^{2}\right)$ d'une impureté de teneur $X$ dans le métal et $\gamma_{\mathbf{B}}$ l'énergie interfaciale.

Cette simple considération laisse entrevoir le fait que les joints spéciaux possédant une énergie relativement basse seront moins sensibles à la ségrégation.

L'interaction impureté-joint de grain a deux origines : élastique, liée à la structure du joint, et électronique (ou chimique), dans la plupart des cas relativement faible dans les métaux.

Des cas extrêmement variés de ségrégation peuvent être envisagés (pour une bibliographie détaillée voir références $[18,163,164])$ :

- Le joint est à faible désorientation et il n'existe pratiquement pas d'interaction entre les atomes, dans ce cas les atomes de soluté peuvent se distribuer avec la même périodicité que la structure du joint; c'est-à-dire que les atomes d'impuretés se localisent dans les sites lacunaires de l'interface constituant alors des défauts ponctuels. Nous ne nous étendrons pas sur cette forme de ségrégation qui, bien entendu, dépend de la structure intrinsèque.

- Les interactions électroniques sont fortes et le joint fortement désordonné ; le joint agit alors comme un site bidimensionnel de formation d'un film c'est-àdire une nouvelle structure qui est souvent l'origine d'un composé tridimensionnel apparaissant entre les deux grains (par exemple film d'oxygène puis oxyde) $[164,165,166]$; il n'y a pas de sites privilégiés dans l'interface. Dans ce cas, il semble que l'orientation du plan du joint contrôle la germination de la nouvelle phase [167]. La ségrégation est alors immédiatement associée à un phénomène de précipitation.

Les deux cas précédents doivent être considérés comme des extrêmes; le plus souvent dans un polycristal métallique, les joints sont à grand angle et les interactions électroniques relativement faibles; dans cette hypothèse de ségrégation seule, nous pensons que le problème essentiel est le lien entre les atomes d'impuretés et les dislocations extrinsèques. On ne peut être que surpris par le manque de travaux à ce sujet si, en faisant le parallélisme avec le cristal, on évoque la grande attraction entre les dislocations de matrice et les impuretés en solution solide. La principale difficulté pour révéler une association, fort probable, entre défaut extrinsèque du joint et impureté ségrégée réside dans les insuffisances actuelles des analyses ponctuelles. Signalons cependant les résultats suivants :

- Des alignements de microprécipités le long des lignes de dislocations extrinsèques ont été mis en évidence dans des aciers austénitiques [168] et fer-chrome [100-174] (Fig. 53). 
- Dans des alliages fer-chrome, des ségrégations de carbone en l'absence de toute précipitation ont été révélées préférentiellement dans des joints contenant une grande densité de dislocations extrinsèques, par autoradiographie à haut pouvoir de résolution du carbone 14 [100-169].

- Enfin, le rôle des dislocations extrinsèques a été précisé dans les travaux récents de Varin [101] : c'est dans les joints spéciaux uniquement que les précipités peuvent nucléer sur les lignes de dislocations extrinsèques, stables dans ce type de joints; le processus n'interviendrait pas dans les joints généraux.

Dans l'association dislocation extrinsèqueimpureté, le principal problème qui se pose est de savoir quel est le phénomène qui entraîne l'autre :

- Les dislocations extrinsèques servent-elles de sites préférentiels de ségrégation pour le soluté ? (auquel cas on comprend mal la localisation des impuretés dans les joints généraux).

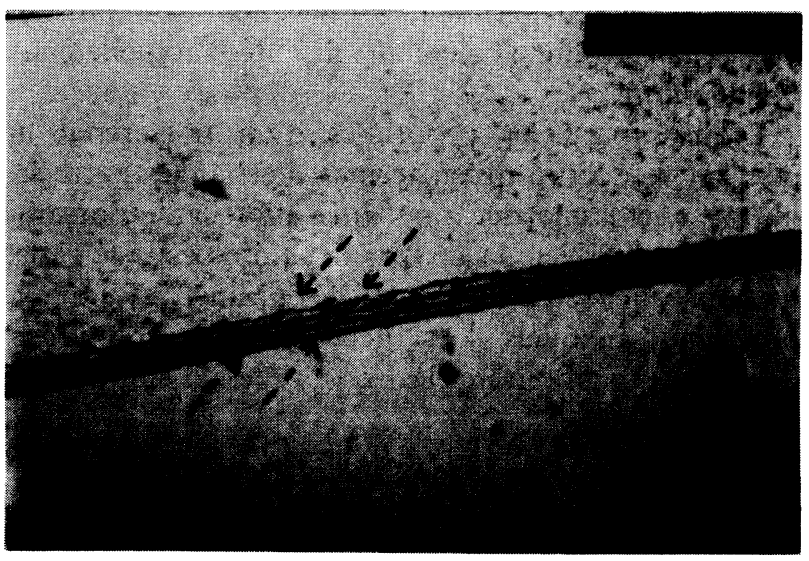

Fig. 53. - Mise en évidence de microprécipités alignés sur les lignes de dislocations extrinsèques dans un alliage fer- $9 \%$ chrome [100].

[Micrograph of carbide precipitates which are decorating extrinsic dislocations in Iron-9\% chromium alloys [100].]

- La présence d'atomes ségrégés dans le joint diminue-t-elle l'énergie de formation des défauts extrinsèques en augmentant ainsi leur probabilité d'apparition et leur quantité selon l'hypothèse de Li [57].

- L'impureté donne-t-elle lieu à un phénomène d'ancrage de la dislocation extrinsèque expliquant ainsi une éventuelle stabilité dans les joints généraux ? A ce propos, signalons que la présence et la stabilité des dislocations extrinsèques ne dépend pas uniquement de la structure du joint mais de la teneur et de la nature des impuretés dans le métal [100-174].

Il est extrêmement important de progresser dans la connaissance des processus de ségrégation intergranulaire lorsqu'on sait :

- d'une part, qu'il suffit de traces minimes d'impuretés dans un joint pour modifier considérable- ment toute propriété de ce joint ; à la limite, l'existence même des interfaces est conditionnée par la pureté du matériau;

- d'autre part, que cette ségrégation est toujours relativement importante (même à teneur globale faible) compte tenu des valeurs généralement élevées des coefficients d'enrichissement $\beta$ [170].

Selon le type de ségrégation, l'approche géométrique ou analytique sera plus ou moins adaptée à l'étude. Dans le cas d'un joint à faible désorientation et d'une faible interaction entre l'impureté et le métal, la ségrégation peut être vue comme une insertion dans les sites possibles des polyèdres d'atomes du solvant dont l'arrangement explique la structure de l'interface. Les développements récents du modèle analytique permettront sans doute de mieux comprendre ce type d'interaction. Dans le cas où il y a interaction électronique forte entre les atomes et précipitation continue rapide dans l'interface, les défauts linéaires intrinsèques ou extrinsèques de celle-ci ne semblent pas influer directement sur le phénomène, aucun modèle n'est spécialement opérant; les méthodes d'analyse tels le S.T.E.M. et la spectroscopie Auger, sont alors privilégiées. Enfin, lorsqu'il y a ségrégation réelle (au-delà de l'insertion seule et avant toute précipitation) et que le rôle des défauts extrinsèques semble déterminant, il est évident que le modèle géométrique reste le mieux adapté pour l'étude de l'interaction impureté-joint de grains.

d) Conclusion. - L'approche géométrique, bien que limitée pour l'étude des propriétés mécaniques, de la migration des interfaces et de la ségrégation et ce d'autant plus que ces propriétés sont fortement interdépendantes, apparaît cependant, dans l'étape actuelle des techniques, comme mieux appropriée que l'approche énergétique à l'étude de ces propriétés. En particulier, la mise en évidence et la caractérisation des dislocations extrinsèques du joint de grains restent le domaine de la microscopie électronique, outil de choix du modèle géométrique.

Des progrès sont possibles en direction du lien avec la structure fine du joint, notamment pour la ségrégation, grâce au développement des techniques suivantes :

- la microscopie électronique à haute résolution [171],

- la sonde associée au microscope à champ ionique [172],

- la détermination des facteurs de structure de l'interface par diffraction des rayons $X$ [173] (possibilité d'utiliser le rayonnement synchrotron de haute intensité),

- les méthodes de simulation à l'ordinateur.

Remarquons que les techniques précédentes sont évidemment intéressantes pour l'étude des propriétés en relation avec la structure intrinsèque du joint. Mais leur développement et leur extension permettent surtout d'envisager l'étude de joints de moins en 
moins spéciaux et de défauts interfaciaux de plus en plus importants. A la limite, ces techniques devraient rendre caduque la distinction que nous avons faite dans les propriétés des interfaces, distinction basée sur des modèles de description du joint auxquels actuellement sont plus ou moins associés des moyens d'étude particuliers.

6. Conclusion. - Cet article constitue une tentative de bilan, dans la profusion des travaux sur les joints de grains depuis quelques années, des recherches qui peuvent être utiles pour le métallurgiste c'est-à-dire pour la connaissance des joints réels. Cet objectif justifie le choix du modèle géométrique comme support à la description de l'interface. Bien entendu, un tel effort de synthèse ne prétend pas être exhaustif.

Rappelons quelques idées essentielles qui se dégagent de ce travail :

- il est nécessaire de connaître la structure d'équilibre du joint pour comprendre ses propriétés, en particulier en termes de défauts par rapport à cet équilibre ;

- la structure du joint consiste en un arrangement d'atomes dont la réalité est difficile à atteindre mais qu'on approche de mieux en mieux par différents modèles. Il convient toujours de distinguer entre la réalité physique de cette structure et sa réalité géométrique et même analytique ;

- la notion de "spécialité " des joints de grains est évolutive en fonction de la signification physique de l'ordre intercristallin qui dépend lui-même du progrès des techniques; il est impossible actuellement d'attribuer rigoureusement à toute interface un caractère spécial ou non spécial;

- un certain parallélisme peut être fait entre la structure du monocristal et la structure du joint de grains : au cristal parfait correspond, dans le modèle géométrique, la structure intrinsèque et, dans le modèle analytique l'ordre à longue distance de polyèdres atomiques dans l'interface; au cristal contenant des défauts ponctuels on peut comparer le modèle analytique qui prévoit l'existence de sites vacants et d'insertion dans des polyèdres; enfin, la structure extrinsèque du joint de grain est identique à la structure écrouie du cristal ;

- ce parallélisme se retrouve au niveau des propriétés : un phénomène qui s'explique sur la base de l'ordre dans le cristal trouve son interprétation à partir de la structure d'équilibre dans l'interface; les propriétés liées à l'existence de défauts ponctuels dans le cristal sont encore difficilement interprétables dans un joint car les défauts équivalents sont peu étudiés, le modèle d'approche possible étant très récent $[9,128]$; enfin, les propriétés qui, dans le grain, dépendent de la présence et du caractère des dislocations, sont, dans l'interface, étroitement liées aux dislocations extrinsèques.

Il semble donc possible actuellement de progresser dans les études des polycristaux puisqu'on dispose de modèles de structures et de réactions des différentes zones qui les constituent : grains et joints de grains; par ailleurs, les interactions entre ces régions peuvent être précisées sur des bicristaux. Mais le problème auquel on se heurte alors c'est la proportion des joints spéciaux et généraux dans les joints dits $a u$ hasard des polycristaux ; en effet, bien que l'importance de la texture des joints de grains sur leurs propriétés n'est pas connue, la comparaison avec les grains laisse supposer une influence notoire; or, la détermination de cette texture est complexe tant du point de vue expérimental que théorique puisque liée à la notion, fortement discutée actuellement, de spécialité.

D'autre part, les propriétés d'un polycristal ne peuvent se décrire qu'en termes d'échanges entre les interfaces et la matrice, une autre question importante est alors soulevée : la texture des joints est-elle cause ou conséquence de la texture des grains ? Autant de problèmes auxquels il est nécessaire de répondre si l'on veut comprendre les propriétés d'emploi des matériaux.

En conclusion, l'étude des joints de grains semble entrer dans une étape nouvelle : c'est l'adéquation des recherches théoriques aux recherches appliquées, non seulement du point de vue des joints eux-mêmes mais surtout de leur importance dans l'ensemble du matériau.

Remerciements. - L'auteur tient à exprimer sa profonde gratitude au Professeur R. W. Balluffi pour l'accueil qu'il lui a réservé dans les départements de "Materials Science and Engineering", tout d'abord, pendant six mois, à l'Université de Cornell puis, plus récemment, au Massachusetts Institute of Technology à Cambridge (U.S.A.) et pour les nombreuses discussions qui lui ont été d'une aide précieuse dans la rédaction de cet article.

\section{Bibliographie}

[1] Bollmann, W., Crystal Defects and crystalline interfaces (Springer Verlag, New York) 1970.

[2] BollmanN, W., J. Microsc. 102 (1974) 233.

[3] Smith, D. A., Pond, R. C., Int. Metal Rev. 205 (1976) 61.

[4] Weins, M. J., Gleiter, H., Chalmers, B., J. Appl. Phys. 42 (1971) 2639

[5] Hasson, G., Boos, J. Y., Herbeuval, I., Biscondi, M., Goux, C., Surf. Sci. 31 (1972) 115.
[6] Pond, R. C., Vitek, V., Proc. Roy. Soc. London A 357 (1977) 453.

[7] Smith, D. A., Pond, R. C., Vitek, V., Acta Met. 25 (1977) 475.

[8] Ashby, M. F., Spaepen, F., Scripta Met. 12 (1978) 193.

[9] Ashby, M. F., Spaepen, F., Williams, S., Acta Met. 26 (1978) 1647.

[10] Pond, R. C., Proc. Roy. Soc. London A 357 (1977) 471. 
[11] Balluffi, R. W., Komem, Y., Schober, T., Surf. Sci. 31 (1972) 68.

[12] Gleiter, H., Chalmers, B., High angle grain boundaries (Pergamon Press, New York) 1972.

[13] SAINFORT, G., Interfaces et surfaces en Métallurgie. Ecole d'été de Métallurgie Physique Gassin (1973), Pub. Trans. Tech.

[14] Goux, C., Can. Met. Quat. 13 (1974) 9.

[15] Sмith, D. A., J. Physique Colloq. 36 (1975) C4-1.

[16] LOBERG, B., NORDEN, H., Grain boundary structure and properties, ed. by G. A. Chadwick and D. A. Smith (Academic Press, New York) 1976, p. 1.

[17] PUMPhreY, P. H., Grain boundary structure and properties, ed. by G. A. Chadwick and D. A. Smith (Academic Press, New York) 1976, p. 139.

[18] Balluffi, R. W., Interfacial Segregation, published by A. S. M. Ohio (1979) p. 193.

[19] Hirth, J. P., Balluffi, R. W., Acta Met. 21 (1973) 928.

[20] Biscondi, M., J. Physique Colloq. 36 (1975) C4-57.

[21] Schober, T., Balluffi, R. W., Phil. Mag. 20 (1969) 511.

[22] Sass, S. L., Tan, T. Y., Balluffi, R. W., Phil. Mag. 31 (1975) 559.

[23] Tan, T. Y., Sass, S. L., Balluffi, R. W., Phil. Mag. 31 (1975) 575.

[24] Goux, C., J. Physique Collog. 36 (1975) C4-111.

[25] Goodhew, P. J., Darby, T. P., Balluffi, R. W., Scripta Met. 10 (1976) 495.

[26] Sadanaka, K., Marcinkowski, M. J., Scripta Met. 7 (1973) 557.

[27] Brandon, D. G., Acta Met. 14 (1966) 1479.

[28] Bollmann, W., Phil. Mag. 16 (1967) 383.

[29] Schober, T., Balluffi, R. W., Phil. Mag. 21 (1970) 109

[30] Warrington, D. H., Bollmann, W., Phil. Mag. 25 (1972) 1195.

[31] Balluffi, R. W., Tan, T. Y., Scripta Met. 6 (1972) 1033.

[32] Balluffi, R. W., GoodheW, P. J., TaN, T. Y., Wagner, W. R., J. Physique Colloq. 36 (1975) C4-17.

[33] Gleiter, H., Proc. of the 6th congress on Electron Microscopy Israel (1976) p. 218.

[34] Balluff, R. W., Woolhouse, G. R., Komem, Y., The nature and behavior of grain boundaries, ed. by $\mathrm{Hsun} \mathrm{Hu}$ (Plenum Press) 1972.

[35] Forwood, C. T., Humble, P., Phil. Mag. 31 (1975) 1011; Phil. Mag. 31 (1975) 1025.

[36] McDonald, R. C., Ardell, A. R., Phys. Status Solidi (a) 18 (1973) 407

[37] IsHIDA, Y., J. Mat. Sci. 7 (1972) 72.

[38] Varin, R. A., Kozubowski, J. A., Grabski, M. W., $J$. Physique 36 (1975) 43.

[39] Schober, T., Balluffi, R. W., Phil. Mag. 24 (1971) 165.

[40] Frank, F. C., Defects in crystalline solids (The Physical Society) 1955.

[41] Pumphrey, P. H., Bowkett, K. M., Phil. Mag. 24 (1971) 225.

[42] Bollmann, W., Michaut, B., Sainfort, G., Phys. Status Solidi A 13 (1972) 637.

[43] Pond, R. C., Ph. D. Thesis University of Bristol (1973).

[44] Pond, R. C., Smith, D. A., Phil. Mag. 36 (1977) 353.

[45] Schober, T., Warrington, Phys. Status Solidi A 6 (1971) 103.

[46] KegG, G. R., Horton, C. A. P., Silcock, J. M., Phil. Mag. 27 (1973) 1041.

[47] Horton, C. A. P., Silcock, J. M., KegG, G. R., Phys. Status Solidi A 26 (1974) 215.

[48] Silcock, J. M., KegG, G. R., Horton, C. A. P., J. Microsc. 102 (1974) 331.

[49] Darby, T. P., Schindler, R., Balluffi, R. W., Phil. Mag. 37 (1978) 245

[50] Hirth, J. P., Acta Met. 22 (1974) 1023.

[51] Pumphrey, P. H., Gleiter, H., J. Microsc. 102 (1974) 349.

[52] Pumphrey, P. H., Gleiter, H., Phil. Mag. 30 (1974) 593.

[53] Pumphrey, P. H., J. Physique Colloq. 36 (1975) C4-23.
[54] Gleiter, H., Scripta Met. 11 (1977) 305.

[55] Balluffi, R. W., Scripta Met. 10 (1976) 913.

[56] Bishop, G. H., Chalmers, B., Scripta Met. 2 (1968) 133.

[57] LI, J. C. M., Trans. AIME 227 (1963) 239.

[58] Bernstein, I. M., Rath, B. B., Met. Trans. 4 (1973) 1545.

[59] Murr, L. E., Met. Trans. 6A (1975) 505.

[60] Ventakesh, E., MurR, L. E., Scripta Met. 10 (1976) 477.

[61] Gleiter, H., Acta Met. 17 (1969) 565.

[62] Pond, R. C., SMith, D. A., Proc. of the 6th congress on Electron Microscopy Israel (1976) p. 500.

[63] HoffmanN, R. E., Acta Met. 4 (1956) 97.

[64] Aust, K. T., Rutter, J. W., Trans. Met. soc. AIME 215 (1959) 119.

[65] Tan, T. Y., Hwang, J. C. M., Goodhew, P. J., Balluffi, R. W., Thin solid films 33 (1976) 1.

[66] Czochralski, J., Z. Physik. Chem. 92 (1918) 473.

[67] Cosandey, F., Kang, S. K., Bauer, C. L., J. Physique Colloq. 36 (1975) C4-53.

[68] Thölen, A. R., Phys. Status Solidi (a) 2 (1970) 537.

[69] Balluffi, R. W., Sass, S. L., Schober, T., Phil. Mag. 26 (1972) 585

[70] Guan, D. Y., Sass, S. L., Phil. Mag. 27a (1973) 1211; Phil. Mag. 27b (1973) 1225.

[71] Warrington, D. H., Grimmer, Phil. Mag. 30 (1974) 461.

[72] Grimmer, H., Bollmann, W., Warrington, D. H., Acta Crystallogr. A 30 (1974) 197.

[73] Schober, T., Balluffi, R. W., Phys. Status Solidi 44b (1971) 103.

[74] Darby, T. P., Balluffi, R. W., Phil. Mag. 36 (1977) 53.

[75] Darby, T. P., BallufFi, R. W., à paraître.

[76] Balluffi, R. W., Communication personnelle.

[77] Schober, T., Balluffi, R. W., Phys. Status Solidi 44b (1971) 115.

[78] LeVY, J., Phys. Status Solidi 31 (1969) 193.

[79] Pumphrey, P. H., Scripta Met. 6 (1972) 107.

[80] Warrington, D. H., Boon, M., Acta Met. 23 (1975) 599.

[81] Balluffi, R. W., Schober, T., Scripta Met. 6 (1972) 697.

[82] Bollmann, W., Phys. Status Solidi (a) 21 (1974) 543.

[83] Ralph, B., Howell, R. R., Page, T. F., Phys. Status Solidi (b) 55 (1973) 641

[84] Hirsch, P., Howie, A., Nicholson, R. B., Pashley, D. W., WhELAN, M. J., Electron Microscopy of thin crystals, Robert E. Krieger (Publishing Company, Huntington, New York) 1977.

[85] SChindler, R., Clemens, J. E., Balluffi, R. W., Communication au $9^{\mathrm{e}}$ Congrès international de Microscopie électronique, Toronto, août 1978.

[86] Loberg, B., Norden, H., Arkiv Fys. 40 (1970) 413.

[87] SChindler, R., Clemens, J. E., BallufFi, R. W., à paraître dans Acta Met.

[88] Gronsky, R., Scripta Met. 11 (1977) 791.

[89] SCHINDleR, R., Communication personnelle.

[90] Jones, A. R., Howell, P. R., Ralph, B., Phil. Mag. 85 (1977) 603.

[91] Priester, L., Balluffi, R. W., J. Microsc. Spectrosc. Elec. 4 (1979) 615.

[92] Clark, W. A. T., Smith, D. A., J. Mat. Sciences 14 (1979) 776.

[93] IshidA, Y., Henderson Brown, M., Acta Met. 15 (1967) 857.

[94] VARIN, R. A., WyrzkowsKi, J. W., LoJKowsKi, W., GRABSKI, M. W., Phys. Status Solidi (a) 45 (1978) 565.

[95] Ishida, Y., Hasegawa, T., Nagata, F., J. Appl. Phys. 40 (1969) 2182

[96] Pumphrey, P. H., Phys. Status Solidi (a) 28 (1975) 545.

[97] Loberg, B., Norden, H., Acta Met. 21 (1973) 213.

[98] Howell, P. R., Jones, A. R., RalPh, B., J. Mater. Sci. 10 (1975) 1351.

[99] Dingley, D. J., Pond, R. C., Acta Met. 27 (1979) 667.

[100] Khalfallah, O., Bouchet, D., Priester, L., Mem. Scient. Rev. Met. à paraître.

[101] Varin, R. A., J. Mater. Sci. 14 (1979) 811. 
[102] Vitek, V., Sutton, A. P., Smith, D. A., Pond, R. C., Phil. Mag. A 39 (1979) 213.

[103] Marcinkowski, M. J., Weng Feng Tseng, DwarakaDASA, E. S., Phys. Status Solidi (a) 22 (1974) 659.

[104] Tunstall, W. J., VII Cong. Internat. Microscopie Electronique, Grenoble 1970, p. 313.

[105] Varin, R. A., Kozubowski, J. A., Grabski, M. W., BuszKowski, R., Phys. Status Solidi (a) 34 (1976) 711.

[106] IsHidA, Y., MoRI, M., UdA, F., Acta Met. 25 (1977) 815.

[107] MurR, L. E., Ventakesh, E., Metallography 11 (1978) 61.

[108] MaRCINKowsKi, M. J., Electron Microscopy and structure of materials, Thomas ed. (Californie Press) 1972.

[109] Ingram, J. C., Strutt, P. R., Wen Shian Tzeng, Phys. Status Solidi (a) 22 (1974) 599.

[110] TuCKer, M. O., Phil. Mag. 19 (1969) 114.

[111] Pumphrey, P. H., Bowkett, K. M., Phys. Status Solidi (a) 2 (1970) 339.

[112] Hancock, P. C., Lorimer, G. W., 7e Congrès de Microscopie électronique, Grenoble 1970, 187.

[113] Young, C. T., Steele, J. H., Lytton, J. L., Met. Trans. 4 (1973) 2081.

[114] Karakostas, T. H., Nouet, G., Bleris, G. L., Hasege, S., Delavignette, $P$.

[115] Bonnet, R., Laufer, E. E., Phys. Status Solidi (a) 40 (1977) 599.

[116] Fontaine, C., Rocher, A., à paraître.

[117] Goux, C., Mem. Scient. Rev. Met. LVIII (1961) 661.

[118] Grimmer, H., Acta Cryst. A 30 (1974) 685.

[119] Bonnet, R., Acta Cryst. A 36 (1980) 116.

[120] Rey, C., SAada, G., J. Physique 38 (1977) 721.

[121] SAADA, G., Acta Met. 27 (1979) 921.

[122] Weatherly, G. C., MoK, T. D., Proceedings of the International Conference on structure and properties of grain boundaries and interfaces. I. B. M. Thomas, J. Watson Research Center, New York (1971).

[123] Sass, S. L., Balluffi, R. W., Phil. Mag. 33 (1976) 703.

[124] Carter, C. B., Donald, A. M., Sass, S. L., Phil. Mag. A 39 (1979) 533.

[125] Harrison, R. J., Bruggeman, G. A., Bishop, G. H., Grain boundary structure and properties, p. 45, ed. by G. A. Chadwick et D. A. Smith (Academic Press, London) 1976.

[126] Simon, J. P., Guyot, P., Phys. Status Solidi (a) 38 (1976) 207.

[127] Bristowe, P. D., SASs, S. L., à paraître dans Acta Met.

[128] Pond, R. C., SMith, D. A., ViteK, V., Acta Met. 27 (1979) 235.

[129] Bernal, J. D., Proc. Roy. Soc. A 280 (1964) 299.

[130] Allen, R. M., Goodhew, P. J., Acta Met. 25 (1977) 1095.

[131] Goodhew, R. J., Tan, T. Y., Balluff, R. W., Acta Met. 26 (1978) 557.

[132] Herring, G., Phys. Rev. 82 (1951) 87.

[133] Masteller, M. S., Bauer, C. L., Acta Met. 27 (1979) 483.

[134] Hwang, J. C. M., Balluffi, R. W., Scripta Met. 12 (1978) 709.

[135] Herbeuval, J., Biscondi, M., Goux, C., Mem. Scient. Rev. Met. 70 (1973) 39.

[136] Martin, G., Perraillon, B., J. Physique Colloq. 36 (1975) C4-165.
[137] Assassa, W., Guiraldenc, P., Beaunier, L., Froment, M., J. Physique Colloq. 36 (1975) C4-225.

[138] Turnbull, D., Hoffman, R. E., Acta Met. 2 (1954) 419.

[139] Benoist, P., Martin, G., J. Physique 36 (1975) C4-213.

[140] Gleiter, H., Acta Met. 27 (1979) 187.

[141] BeEL ER, J. R., DAHL, R. E., BOURQUIN Jr and R.D., J. Physique Colloq. 36 (1975) C4-97.

[142] FuIs, J., J. Brit. Corros. 3 (1968) 182.

[143] Froment, M., J. Physique Colloq. 36 (1975) C4-371.

[144] Boos, J., Goux, C., Communication au Congrès International "l'hydrogène dans les métaux " Châtenay-Malabry 1 (1972) 133.

[145] Beaunier, L., Thèse, Paris (1979).

[146] Beaunier, L., Froment, M., Electrochem. Acta, à paraître.

[147] Pond, R. C., Smith, D. A., Southerden, P. W. J., Phil. Mag. A 37 (1978) 27.

[148] Lagarde, P., Biscondi, M., J. Physique Colloq. 36 (1975) C4-297.

[149] Watanabe, T., Kuriyama, N., Karashima, S., Proc. ICSMA 4 Nancy (1976) 383.

[150] Biscondi, M., Goux, C., Mem. Scient. Rev. Met. 65 (1968) 167.

[151] Michaut, B., Thèse Grenoble (1971).

[152] Watanabe, T., Davies, P. W., Phil. Mag. A 37 (1978) 649.

[153] HirTh, J. P., Met. Trans. 3 (1972) 3047.

[154] CAHN, J. W., PAN, J. D., Balluffi, à paraître.

[155] Dimitrov, O., J. Physique Colloq. 36 (1975) C4-319.

[156] Guillope, M., PoIrier, J. P., Acta Met., à paraître.

[157] Howell, P. R., Jones, A. R., Horsewell, A., RalPh, B., Phil. Mag. 33 (1971) 21.

[158] Rutter, J. W., Aust, K. T., Acta Met. 13 (1965) 181.

[159] LUCKe, K., Can. Met. Quarter. 13 (1974) 261.

[160] Drolet, J. P., Galibois, A., Met. Trans. 2 (1971) 53.

[161] Simpson, C. J., Winegard, W. C., Aust, K. T., Grain boundary structure and properties, ed. by G. A. Chadwick and D. A. Smith (Academic Press, New York) 1976, p. 201.

[162] Haessner, F., J. Physique Colloq. 36 (1975) C4-345.

[163] Balluffi, R. W., à paraître.

[164] Hondros, E. D., Grain boundary structure and properties, ed. by G. A. Chadwick and D. A. Smith (Academic Press, New York) 1976, p. 265.

[165] Hondros, E., Seah, M. P., Met. Trans. 8A (1977) 1363.

[166] Guttmann, M., Met. Trans. 8A (1977) 1383.

[167] Butler, E. P., Swann, P. R., Acta Met. 24 (1976) 343.

[168] Jones, A. R., Howell, P. R., Page, T. F., RalPh, B., Proc. 4th Bolton Landing Conf. on Grain Boundaries in Engineering Materials.

[169] Bouchet, D., thèse Orsay, 1978.

[170] Hondros, E. D., J. Physique Colloq. 36 (1975) C4-117.

[171] KrivaneK, O. L., Isoda, S., Kobayaski, K., Phil. Mag. 36 (1977) 931.

[172] Hall, T. M., Wagner, A., Seidman, D. N., J. Phys. E 10 (1977) 884.

[173] SAss, S. L., Proc. of the sixth European Congress on Electron Microscopy, Vol. 1, ed. by D. G. Brandon (Tal International Publ. Co., Israel) 1976, p. 221.

[174] Khalfallah, O., Priester, L., Scripta Met. (à paraître). 